\title{
Organised Functional Liquids for Photon Upconversion
}

\author{
by \\ Lia Catherine van den Kerkhof
}

A thesis

submitted to the Victoria University of Wellington

in partial fulfilment of the requirements for the degree of

Master of Science with Honours

Victoria University of Wellington

2015 



\begin{abstract}
Photon upconversion is a process by which lower energy photons are converted to higher energy photons, which can be achieved by the interaction of two triplet excited states. This process holds potential for wavelength shifting solid films in photovoltaic cells. Not all wavelengths emitted by the sun have sufficient energy to be utilized by such devices. Typical solar cells have a band gap of around $1 \mu \mathrm{m}$, however there is a significant amount of energy output by the sun that falls below this threshold. Upconversion could lead to more efficient use of energy by converting these lower energy wavelengths to wavelengths that could be directly absorbed by the solar panel. Upconversion has thus far been harnessed in solution, where diffusion is the limiting factor for the efficiency of the process. However, for technological applications it would be better to create thin solid films. In these films, molecules would have to be brought within the distance on which upconversion occurs, as the process would no longer be defined by diffusion. One way to achieve this would be to create liquid crystalline derivatives of upconversion emitter molecules. This would provide ordering in the system, which would enhance electronic coupling and bring molecules within the scale on which upconversion occurs.

The work of this thesis has focused on the synthesis of these organised functional liquids: liquid crystals of common upconversion emitter molecules. 9,10-diphenylanthracene (DPA) and 9,10-bis(phenylethynyl)anthracene (BPEA) are popular emitter molecules, and derivatives of these molecules were synthesized. A variety of alkyl chains were attached with or without phenyl linkers. The alkyl chains would provide entropy to the system in order to induce the formation of liquid crystalline phases. The resulting phase behaviour of
\end{abstract}


these derivatives was studied using differential scanning calorimetry (DSC) and polarised optical microscopy (POM).

Eight novel derivatives of DPA and BPEA were synthesized. New information was gained as to the requirements of inducing liquid crystallinity in these dye molecules. Direct addition of chains symmetrically to the central dye molecules did not result in the formation of liquid crystalline phases. Through extension of the central core by an extra phenyl ring a liquid crystalline behaviour was observed. A synthesized derivative of DPA exhibited extreme supercooling, which is one of a few derivatives of 9,10diphenylanthracene to exhibit a liquid state at room temperature. A derivative of BPEA was synthesized that exhibited formation of a mesophase (liquid crystal phase). These two derivatives were investigated for potential use as a material for upconversion. 


\section{Acknowledgements}

I would like to thank my three supervisors, Dr. Justin Hodgkiss, Prof. Kate McGrath and Prof. John Spencer, whose help and support have been invaluable to me over the past two years and whom I have been priviledged to work with.

I would like to thank my parents, Mary and Eugene who have encouraged and supported me throughout these two years, without which I wouldn't have come as far as I have.

Thank you to my three groups; the Hodgkiss, McGrath and Spencer groups for your friendship and support. Special thanks go to Dr. Kathryn Allan for all your advice and synthesis of certain compounds. Thanks go to Jackie and Teresa for lending me equipment. I would also like to thank the Curtis-Gordon Research Scholarship in Chemistry for funding.

In the categories of random skills I have gained, I have been known to do ten columns in three weeks, there are people who would never have thought I would say that and I have pressed emergency stop buttons on fumehoods with my head (accidentally). I have synthesized a great number of fluorescent samples and have enjoyed showing them off, thank you to those who have appreciated them with me.

To my friends - thank you for the cups of tea and hot chocolate and chocolate eating sessions that we have had over the past couple of years, these are the exact requirements of keeping a postgraduate student sane. Thanks go to all my friends at dancing, a great many dances have contributed to the survival of this thesis. 
Masters has required a great amount of hard work and dedication and I am so proud of what I have achieved and the skills I have gained.

To all those others who have supported and helped me, you know who you are and I am extremely grateful. 


\section{Table of Contents}

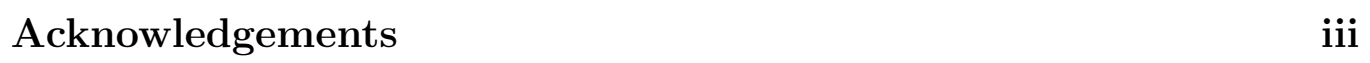

$\begin{array}{ll}\text { Table of Contents } & \mathbf{v}\end{array}$

List of Figures viii

List of Schemes xii

List of Tables xiv

Glossary

1 Outline 1

1.1 Research Proposal . . . . . . . . . . . . . . . . 5

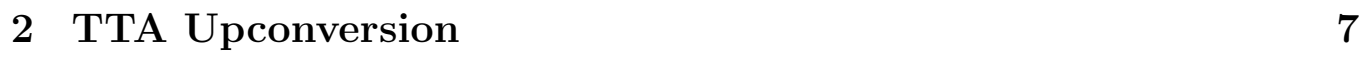

2.1 Background . . . . . . . . . . . . . . . . . . . . . . . . 7

2.2 TTA Upconversion in Solution . . . . . . . . . . . . . . . . . 12

$2.2 .1 \quad \mathrm{DPA} \ldots \ldots \ldots \ldots \ldots \ldots$

$2.2 .2 \quad$ BPEA $\ldots \ldots \ldots \ldots \ldots$

2.3 TTA Upconversion in Solids . . . . . . . . . . . . . . . . . 20

3 Liquid Crystals 25

3.1 Background . . . . . . . . . . . . . . . . . . . 26

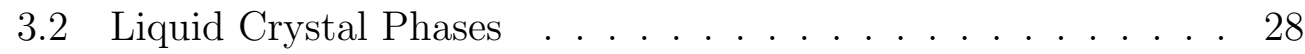




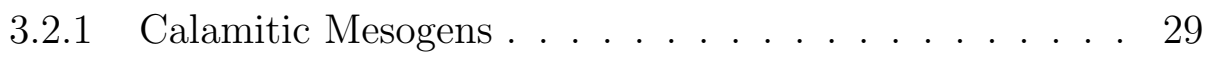

3.2 .2 Discotic Mesogens . . . . . . . . . . . . . . . . 30

3.3 Anthracene Derived Liquid Crystals . . . . . . . . . . . . . . . 32

$3.4 \quad 9,10$-Substitution $\ldots \ldots \ldots 33$

3.4 .1 DPA Derivatives . . . . . . . . . . . . . . . . . . . . 33

3.4 .2 BPEA Derivatives . . . . . . . . . . . . 36

\begin{tabular}{|lll}
4 & DPA Derivatives & 41
\end{tabular}

4.1 Synthesis of Dicatenar DPA Derivatives . . . . . . . . . . . . . 42

4.2 Phase Behaviour of Dicatenar DPA Derivatives . . . . . . . . 44

4.3 Synthesis of Hexacatenar DPA Derivatives . . . . . . . . . . 47

4.4 Phase Behaviour of Hexacatenar DPA Derivatives . . . . . . . 51

4.5 Conclusions . . . . . . . . . . . . . . . . . . . . . . . . 55

$\begin{array}{lll}5 & \text { BPEA Derivatives } & 57\end{array}$

5.1 Synthesis of Dicatenar BPEA Derivatives . . . . . . . . . . . 58

5.2 Phase Behaviour of Dicatenar BPEA Derivatives . . . . . . . . 61

5.2.1 Phase Behaviour of the Dicatenar BBPEA Derivative . 64

5.3 Conclusions . . . . . . . . . . . . . . . . . . . . . . 67

$6 \quad$ Spectroscopy of DPA and BPEA Derivatives 69

6.1 Upconversion in Solution . . . . . . . . . . . . . . . 70

6.2 Spectroscopy of DPA Derivatives . . . . . . . . . . . . . . 79

6.2 .1 Solution Phase Spectroscopy . . . . . . . . . . . . . . . 79

6.2.2 Hexacatenar Decyl DPA Derivative Thin Film Study . 82

6.3 Spectroscopy of BPEA Derivatives . . . . . . . . . . . 86

6.3 .1 Solution Phase Spectroscopy . . . . . . . . . . . . . . 86

6.3.2 Thin Film Spectroscopy of the BBPEA Derivative . . . 89

6.4 Conclusions . . . . . . . . . . . . . . . . . . . . . . 93

\begin{tabular}{lll}
\hline & Conclusions and Future Work & 95
\end{tabular}

\begin{tabular}{lll}
\hline 8 & Experimental & 97
\end{tabular} 
$8.1 \quad$ Synthetic Methods . . . . . . . . . . . . . . . . . . . . . . . 97

$8.1 .1 \quad$ Synthesis of Dicatenar DPA Derivatives. . . . . . . . . 98

8.1 .2 Synthesis of Hexacatenar DPA Derivatives . . . . . . . 101

8.1 .3 Synthesis of Dicatenar BPEA Derivatives . . . . . . . . 105

8.2 Liquid Crystal Techniques . . . . . . . . . . . . . . . . . . 108

$8.2 .1 \quad$ Differential Scanning Calorimetry (DSC) . . . . . . . . 108

8.2 .2 Polarised Optical Microscopy (POM) . . . . . . . . . . 109

8.3 Cholesteryl Benzoate - A Worked Example . . . . . . . . . . . 111

8.4 Spectroscopic Methods . . . . . . . . . . . . . . . . . . . . 113

\begin{tabular}{ll}
\hline References & 114
\end{tabular} 


\section{List of Figures}

1.1 Solar Cell Threshold . . . . . . . . . . . . . . . . . . . . . . . 2

1.2 Solar Spectrum . . . . . . . . . . . . . . . . . . . . . . . 2

2.1 Stokes Shift $\ldots \ldots \ldots \ldots$

2.2 Photophysical Processes of Upconversion . . . . . . . . . . . . 9

2.3 Dexter Triplet-Triplet Energy Transfer . . . . . . . . . . . . . 10

2.4 Common Sensitizers and Emitters Used in Upconversion . . . 14

$2.5 \quad \mathrm{DPA} \ldots \ldots \ldots \ldots \ldots$

2.6 Time Evolution of Various Species Imaged with a Streak Camera 18

$2.7 \quad$ Fluorescence of 9,10-Bis(phenylethynyl)anthracene . . . . . . . 19

2.8 BPEA $\ldots \ldots \ldots \ldots \ldots$

2.9 The Effect of Liquid Crystal on Exciton Diffusion in Solid Films 23

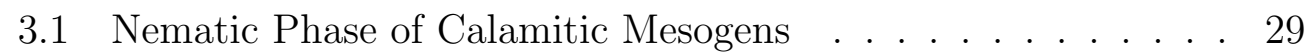

3.2 Smectic Phase of Calamitic Mesogens . . . . . . . . . . . . . . 30

3.3 Phases of Discotic Mesogens . . . . . . . . . . . . . . . . . . . 31

3.4 Substitution Positions of Anthracene . . . . . . . . . . . . . . 32

3.5 Generic Tolane Structure . . . . . . . . . . . . . . . . . . . . . 36

4.1 DSC Plot for Dicatenar DPA Derivatives . . . . . . . . . . . . 44

4.2 POM Images of Dodecyl and Hexadecyl Dicatenar DPA deriva-

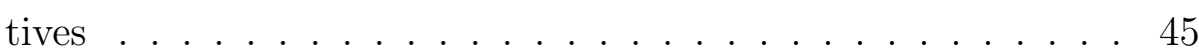

4.3 POM Images of the Hexadecyl Dicatenar DPA Derivative . . . 46

4.4 DSC Plot for 9,10-Bis(3,4,5-tri(decyloxy)phenyl)anthracene . . 51 
4.5 DSC Plot of 9,10-Bis(3,4,5-tri(hexadecyloxy)phenyl)anthracene 54 4.6 POM Images of 9,10-Bis(3,4,5-tri(hexadecyloxy)phenyl)anthracene 55

5.1 DSC Plot for 9,10-Bis(4-(octyloxy)phenylethynyl)anthracene . 62

5.2 DSC Plot for 9,10-Bis(4-(hexadecyloxy)phenylethynyl)anthracene 63

5.3 DSC Plot for 9,10-Bis(4'-(octyloxy)biphenyl-4-ylethynyl)anthracene 65

5.4 POM Images of the Nematic Phase of 9,10-Bis (4'-(octyloxy)biphenyl4-ylethynyl)anthracene . . . . . . . . . . . . . 66

5.5 Mosaic Crystalline Solid of 9,10-Bis(4'-(octyloxy)biphenyl-4ylethynyl)anthracene . . . . . . . . . . . 66

6.1 Absorption and Emission Spectra of DPA and PdOEP . . . . 72

6.2 Upconversion of DPA and PdOEP . . . . . . . . . . . . . 73

6.3 Power Dependence . . . . . . . . . . . . . . . . . . . . . . 73

6.4 Square-Root Intensity against Power . . . . . . . . . . . . . . 74

6.5 Absorption and Emission Spectra of BPEA and PdOEP . . . 76

6.6 Upconversion of BPEA and PdOEP . . . . . . . . . . . . . 77

6.7 Emission Spectrum of a Solution of BPEA and PdOEP . . . . 78

6.8 Absorption Spectra of Dicatenar DPA Derivatives . . . . . . . 79

6.9 Emission Spectra of Dicatenar DPA Derivatives . . . . . . . . 80

6.10 Absorption Spectra of Hexacatenar DPA Derivatives . . . . . 81

6.11 Emission Spectra of Hexacatenar DPA Derivatives . . . . . . . 82

6.12 Absorption Spectra of the Hexacatenar Decyl DPA Derivative in a Thin Film . . . . . . . . . . . . . . . . . . . . . . . 83

6.13 Emission Spectrum of the Hexacatenar Decyl DPA Derivative in a Thin Film . . . . . . . . . . . . . . . . . . . . . . . . 85

6.14 Absorption of BPEA Derivatives . . . . . . . . . . . 87

6.15 Emission Spectra of BPEA Derivatives . . . . . . . . . . . . . 88

6.16 Absorption Spectra of the Liquid Crystalline Derivative . . . . 90

6.17 Emission Spectra of the Liquid Crystalline Derivative . . . . . 91

6.18 POM Images of Liquid Crystal Thin Film Blended with Sen-

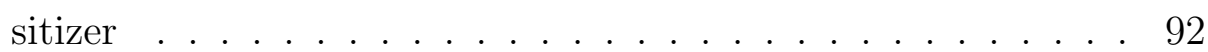


$8.1 \quad$ A DSC Machine . . . . . . . . . . . . . . . . . . . . . . . . . . 109

8.2 Polarised Optical Microscope _. . . . . . . . . . . . . . . . . 110

8.3 Cholesteryl Benzoate . . . . . . . . . . . . . . . . . . . . 111

8.4 Cholesteryl Benzoate at $130^{\circ} \mathrm{C}$. . . . . . . . . . . . . . . 111

8.5 Smectic and Cholesteric Phases of Cholesteryl Benzoate. . . . 112 


\section{List of Schemes}

3.1 Liquid Crystals . . . . . . . . . . . . . . . . . . . . . . . 27

$\begin{array}{lll}3.2 & \text { Double bonded vs. Single Bonded Molecules }\end{array}$. . . . . . . . . . . 28

3.3 Liquid Crystalline Derivatives of DPA . . . . . . . . . . . . . 34

3.4 Liquid Crystalline Derivatives of BPEA . . . . . . . . . . . . . 37

3.5 Liquid Crystalline Derivatives of BBPEA . . . . . . . . . . . . 39

4.1 Synthesis of Dicatenar Derivatives of DPA . . . . . . . . . . . 43

4.2 Synthesis of 3,4,5-Trimethoxyphenyl Boronic Acid Pinacol Ester 48

$4.3 \quad$ Synthesis of Hexacatenar Derivatives of DPA . . . . . . . . . . 50

5.1 Synthesis of 9,10-Bis(4-(octyloxy)phenylethynyl)anthracene . . 60

$5.2 \quad 9,10$-Bis(4-(hexadecyloxy)phenylethynyl)anthracene . . . . . . 61

$5.3 \quad 9,10$-Bis $\left(4^{\prime}\right.$-(octyloxy)biphenyl-4-ylethynyl)anthracene . . . . . 64 


\section{List of Tables}

\begin{tabular}{|c|c|}
\hline 2.1 & Excitation and Emission Wavelengths of Common Sensitizers \\
\hline & Used with DPA \\
\hline 2.2 & Excitation and Emission Wavelengths of Common Sensitizers \\
\hline & Used with BPEA \\
\hline 4.1 & Melting and Crystallisation Temperatures of Dicatenar DPA \\
\hline & $\ldots \ldots \ldots$ \\
\hline 4.2 & Melting and Crystallisation Temperatures for 9,10 -Bis $(3,4,5$ - \\
\hline & tri(hexadecyloxy)phenyl)anthracene . \\
\hline
\end{tabular}




\section{Glossary}

Anisotropic Directionally dependent.

Birefringence The rotation of plane polarised light by a material with multiple refractive indices.

Catenar The number of long alkyl tails attached to the central rigid core of a mesogen.

Emitter The dye molecule in the upconversion process which receives energy from the sensitizer, which it then emits at higher energy through fluorescence.

Fluorescence quantum yield The number of photons that decay via fluorescence out of the number of incident photons that were absorbed.

Mesogen A molecule which exhibits the formation of thermotropic liquid crystal phases.

Mesomorphic An intermediate phase between that of a solid and a liquid, which is fluid and exhibits long range ordering.

Phosphorescence Emission of electromagnetic radiation from a triplet state of a different spin state to the ground singlet state.

Q-band One of the most intense bands of absorption for a porphyrin species typically corresponding to a $\pi-\pi^{*}$ transition.

Sensitizer Typically a heavy metallated species which transfers energy to the emitter from its excited triplet state.

Singlet State An excited state where the spin of the excited electron and the electron left behind are of opposite spins. 
Spin-orbit coupling The interaction of a particle's spin with its motion, which causes shifts in an electron's atomic energy levels due to the interaction between the electromagnetic interaction between the electron's spin and the magnetic field generated by its orbit around the nucleus.

Triplet state An excited state where the spin of the excited electron and the electron left behind are of the same spin.

Upconversion The process by lower energy is converted to higher energy. This is achieved through the combination of two photons in a non-linear medium to produce one photon of higher energy. 


\section{Chapter 1}

\section{Outline}

Photon upconversion is the conversion of low energy photons to higher energy photons. One way to realise upconversion involves the interaction of two triplet excited states, giving rise to one singlet excited state which emits higher energy photons. This process if harnessed in solid films could be used in wavelength shifting films in solid-state lasers or photovoltaic devices. In the case of the latter, upconversion could lead to more efficient use of energy by using more fully the solar spectrum. 1 Photovoltaic devices require a certain level of energy before charge separation and generation of photocurrent can occur. Not all the wavelengths emitted by the sun have sufficient energy to be used by such devices (Fig. 1.1).

Typical silicon solar cells have a band gap of around $1 \mu \mathrm{m}$, however there is a significant amount of energy output by the solar spectrum that falls below this threshold (Fig. 1.2). The Schockley-Queisser limit outlines the maximum theoretical efficiency of $33.7 \%$ for an $\mathrm{p}-\mathrm{n}$ type silicon solar cell. $\stackrel{3}{[}$ 


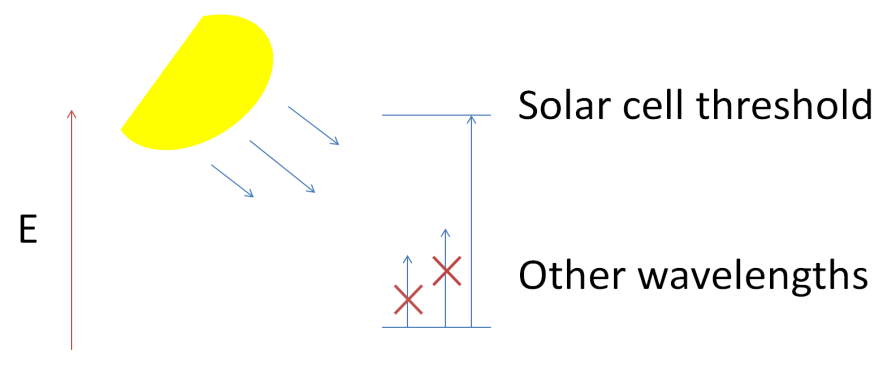

Fig. 1.1 Illustration of the energy requirements of a solar cell.

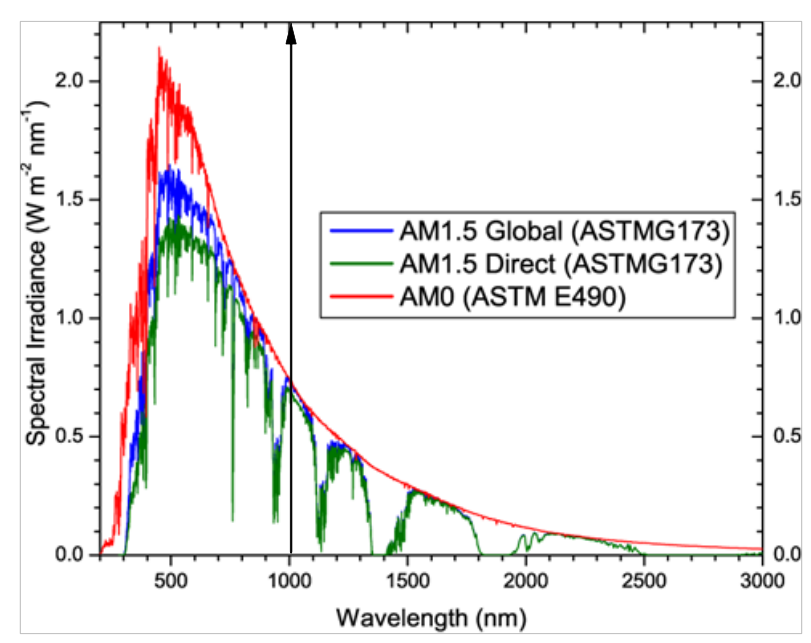

Fig. 1.2 Spectrum of the solar energy output by the sun, where the green curve represents that obtained on earth. The arrow at $1000 \mathrm{~nm}$ illustrates the bandgap of a typical solar cell. ${ }^{2}$ 
This efficiency should be considered as the maximum solar conversion of light into electricity out of all incident light on the solar panel, in an AM 1.5 solar spectrum. The low efficiency is due to three main sources of energy loss: blackbody radiation, radiative recombination and spectrum losses. The first two factors are hard to control within the solar cell. Blackbody radiation is emitted by any material that is not at $0 \mathrm{~K}$. This energy cannot be captured by the cell and so is lost. Radiative recombination in solar cells occurs when separated electrons and holes recombine which results in a loss of electrical current production. Spectrum losses occur as only photons with more energy than the band gap can create a photo-electron charge carrier. This loss accounts for around $33 \%$ of the incident light, which is a significant contribution to the inefficiency of $\mathrm{p}$-n type single junction solar cells. ${ }^{3}$

Upconversion offers the potential to upconvert low energy photons to those of sufficient energy to reach this $1 \mu \mathrm{m}$ threshold, increasing the efficiency of the solar cell and offering to extend the Shockley-Queisser limit. Previous research has focused on observing upconversion in solution. Common emitters such as 9,10-diphenylanthracene (DPA) and 9,10-bis (phenylethnyl)anthracene (BPEA) and sensitizing agents such as heavy metal-containing porphyrin or phthalocyanin rings have been used. ${ }^{4}$ In solution the process requires long-lived and mobile triplet states. It would be better if this process could be harnessed in thin films and solid state materials for the practical advantages that they offer. However there is no sure or straight forward way to control the interaction between these molecules which determines electronic coupling.

Organised functional liquids such as liquid crystals offer the ability to control the way in which molecules self-assemble. In the case of liquid crystals, their interaction can be manipulated through altering the shape and chemical functionalities of the molecules. Their self-assembly allows them to line up within the length scale on which upconversion occurs. These molecules 
are then both within the distance $(10 \AA)$ and arranged in such an orientation which could help upconversion take place more efficiently in solid films.

In the application of a material which can facilitate upconversion, it would be better that the process could be harnessed in thin films. The use of liquids have posed problems in dye sensitized solar cells where ineffective sealing of the electrolytic liquid resulted in leakage over the required lifetime of the solar panels (10-30 years). ${ }^{7}$ The ultimate goal of this research was to synthesize liquid crystalline derivatives based on DPA and BPEA cores that could be used in upconverting solid films which would ultimately be printed as solid films. Ultimately, the material would be heated to the mesomorphic temperature and quickly 'set' in the assembly of molecules as per the liquid crystalline phase and then quickly solidified. The temperature at which the liquid crystalline phase forms must be higher than the temperature that the sun can induce, so the precise assembly of molecules cannot be undone. However, in this thesis preliminary spectroscopic measurements will be done in the liquid phase and not 'set' in a solid state.

This novel research has focused on the synthesis of common emitter molecules that are novel derivatives of DPA and BPEA. The synthesized derivatives incorporate common liquid crystal inducing groups. The molecules were characterised and investigated for the presence of mesomorphic behaviour (liquid crystalline behaviour). A library of molecules were synthesized, building on previous research ${ }^{[8] 9}$ and gave new insight the certain requirements of mesomorphic behaviour for molecules based on BPA and BPEA.

In addition the spectroscopic properties of these compounds were investigated for their potential as materials for use in upconversion. Preliminary studies on how the inclusion of the sensitizer altered these materials were carried out. 


\subsection{Research Proposal}

The goal of this research was to synthesize novel emitter compounds based on common upconversion emitter molecules and study the resulting phase behaviour and spectroscopic properties of these derivatives.

The aims outlined in my research proposal were to:

- synthesize of a variety of anthracene derived mesogens based on the structures of common emitters,

- investigate and characterise the resulting phase behaviour and transition temperatures of the molecules,

- investigate the possibility of upconversion using a common metal complex sensitizer, palladium octaethyl porphyrin (PdOEP), in combination with any liquid crystal mesogens or organised liquids synthesized.

In the next two chapters Triplet-Triplet Annihilation (TTA) upconversion and liquid crystals are introduced. The following two chapters will detail the synthesis of the upconversion emitter derivatives, and the resulting phase behaviour of these substances. Finally the spectroscopy on these derivatives wil be explored. All experimental information will be detailed in the final chapter of this thesis. 


\section{Chapter 2}

\section{Triplet-Triplet Annihilation (TTA) Upconversion}

\subsection{Background}

Upconversion is the process by which lower energy photons are converted to higher energy photons. This is a counter-intuitive process, in comparison to fluorescence where loss of vibrational energy in the excited state results in the emission of lower energy photons. Upconversion involves the absorption of photons at a lower-energy wavelength and emission of photons at a wavelength of higher energy. This is referred to as a delayed anti-Stokes shift (Fig. 2.1, 10

The key to this process is that the triplet excited state of the emitting molecule is half the energy of its singlet excited state. ${ }^{11] 13}$ A dimer complex is formed when two of these triplet states come together. Triplet-triplet annihilation (TTA) occurs where energy of the excited state of one molecule 
is transferred to the other, forming one molecule of a singlet excited state and one molecule in the ground state. This is a spin allowed process, as two triplets of opposite spin are paired.
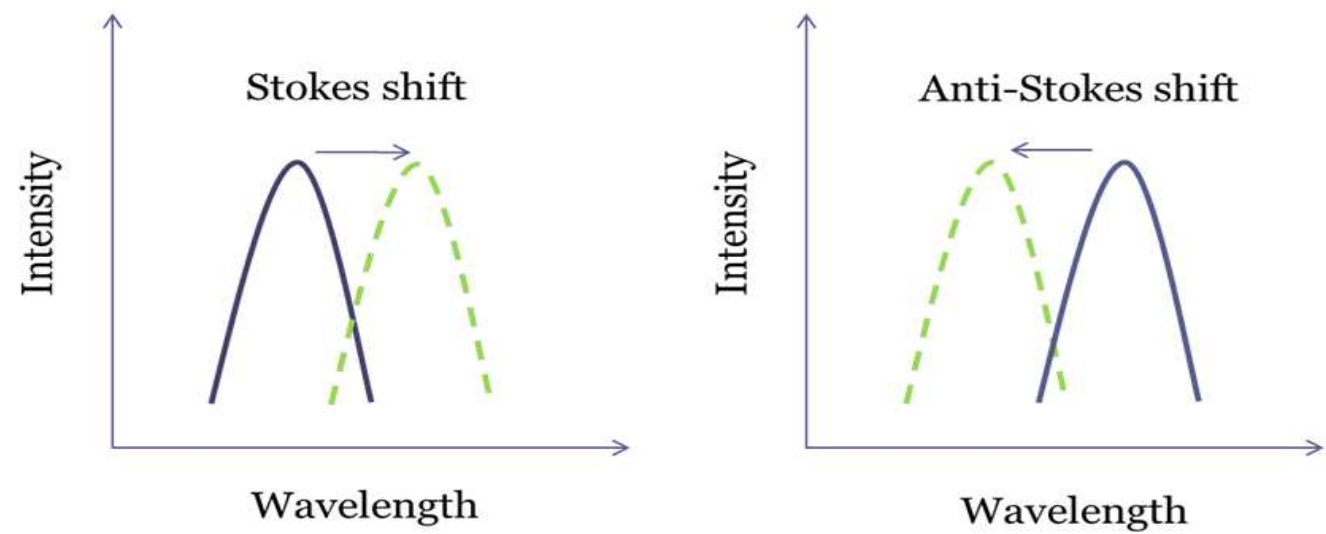

Fig. 2.1 Schematic of absorption and emission spectra occurring in both fluorescence (left) and upconversion (right).

Fluorescence then occurs from the excited state that is almost double the energy of the triplet state. This fluorescence is said to be 'delayed' owing to the time needed for buildup of a significant population of singlet states before upconversion can occur (which occurs on nanosecond timescales and longer).

A sensitizing agent or a "sensitizer" is used to give rise to a sufficient population of triplet states. The sensitizer is commonly a porphyrin or phthalocyanin complex containing a heavy metal such as Ru, Ir, Pd or Pt.114 18 The heavy metal enhances spin-orbit coupling which yields intersystem crossing (ISC) efficiencies near unity. ${ }^{[19}$ This triplet state can transfer energy to the emitting molecule through an allowed triplet-triplet energy transfer (TTET) process (Fig. 2.2). The triplet excited state of the emitter must be lower in energy than the sensitizer to encourage the transfer of energy. 


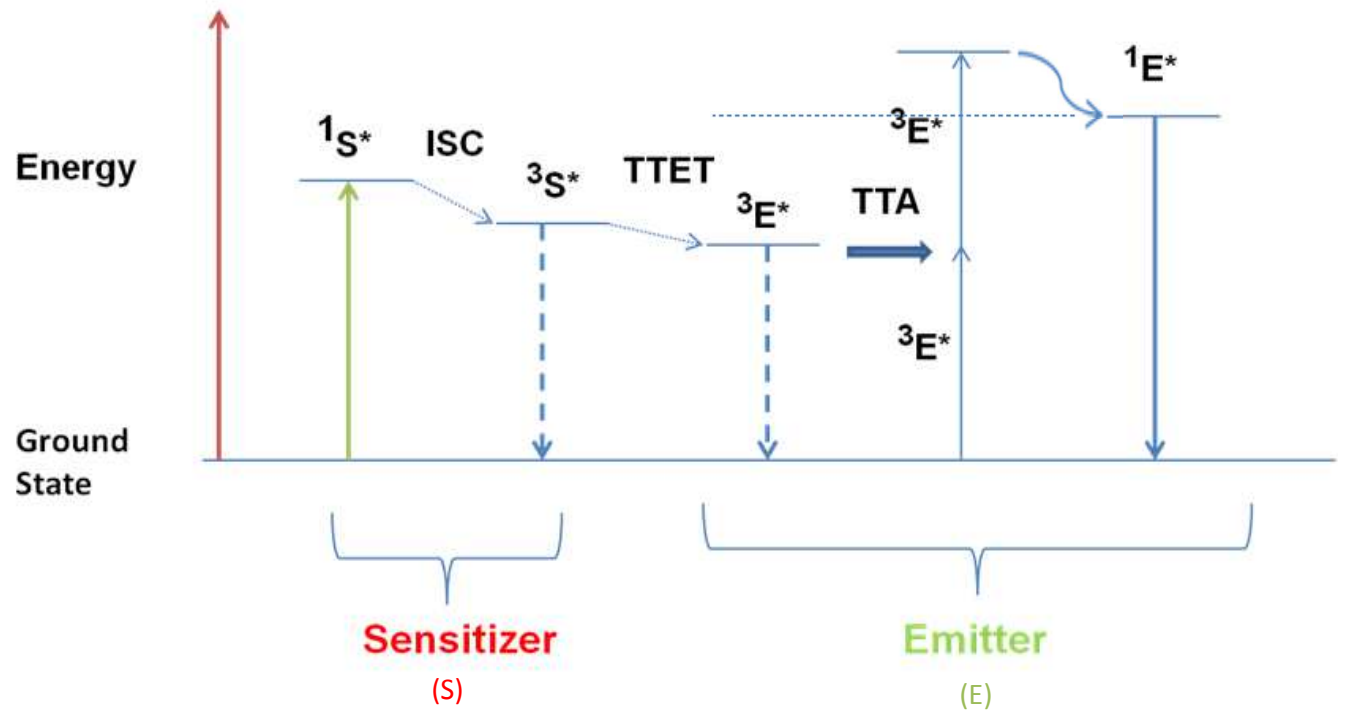

Fig. 2.2 Schematic of the photophysical processes occurring in upconversion.

As a consequence of the high exchange interaction, polyaromatic $\pi$ systems contain a triplet state that is both half the energy of its own singlet state and is lower than that of the sensitizer. They have slow intersystem crossing, which also prevents triplet repopulation. In comparison, the metallated sensitizer has very low exchange energy, the triplet state is more similar in energy to the singlet state, and this results in fast intersystem crossing, which serves as a reliable reservoir of long-lived triplet states.

TTET and TTA, the photophysical processes occurring in upconversion, both involve a Dexter type energy transfer. Dexter type energy transfer is different from Förster energy transfer. Förster energy transfer involves resonant transfer of energy in which the emission from one excited state is used to excite another state. ${ }^{20}$ Dexter type energy transfer involves the transfer of an electron, which is a spin allowed process. $\stackrel{21}{21}$ For this type of exchange to occur short distances between molecules or the ability for molecules to move freely towards each other is required so that the involved orbitals can 
overlap. Thus, this process is termed short-range or collisional transfer.

TTET is considered Dexter transfer in the traditional sense, where an excited triplet state can interact with a singlet ground state. The triplet state electron $\left({ }^{3} \mathrm{~S}^{*}\right)$ is transferred to the triplet state of the accepting molecule $\left({ }^{3} \mathrm{E}^{*}\right)$ Fig. 2.3), while an electron ground state of the acceptor $\left({ }^{1} \mathrm{E}\right)$ is transferred to the sensitizer $\left({ }^{1} \mathrm{~S}\right)$.

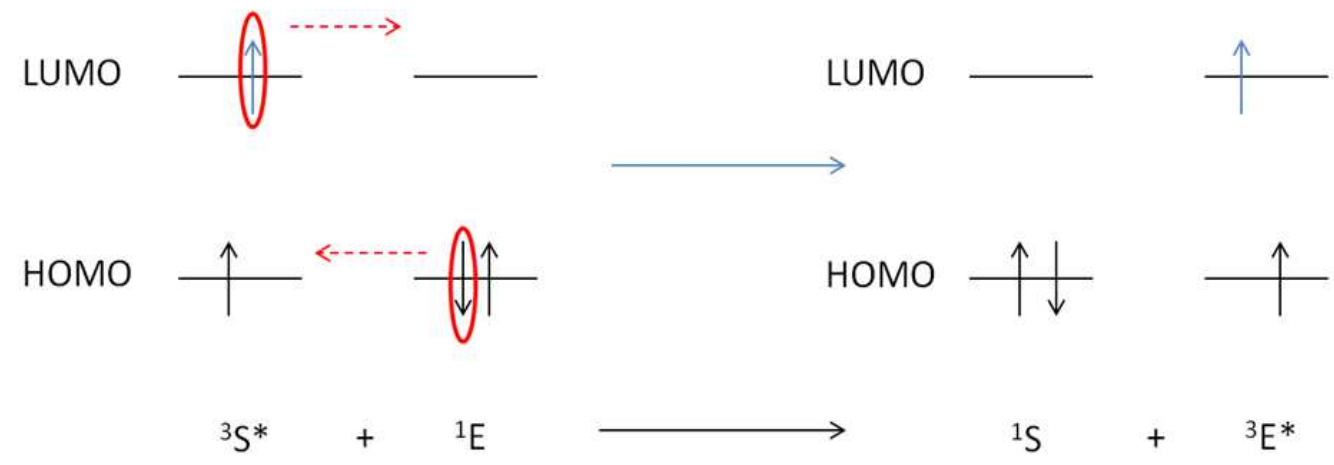

Fig. 2.3 Schematic of Dexter triplet-triplet energy transfer (TTET).

In the case of TTA, two excited triplet states interact to form one singlet state, as stated above. The rate constant of Dexter energy transfer decays exponentially with respect to increasing distance between the two molecules. The simplified rate constant of exchange transfer is depicted below:

$$
k_{\text {Dexter }}=K J \exp \left(-2 R_{D A} / L\right)
$$

where $\mathrm{J}$ is the normalized spectral overlap integral, $\mathrm{K}$ is an experimental factor, $\mathrm{R}$ is the distance between the sensitizer and the emitter and $\mathrm{L}$ is their effective Bohr radius which measures the spatial extent of the donor and acceptor wavefunctions. ${ }^{6 / 21 \mid 22}$ It must be noted that this is a simplified equation, and the more complex equations can be found in the cited references. $\frac{6222}{6}$ 
Due to the exponential nature of this equation the rate is fastest at short distances of the donor and acceptor molecules and usually occurs within $10 \AA .516$ This has large implications for the application of upconversion in materials. In solution molecules must have diffusional mobility or high concentration such that molecules collide for the electron transfer to occur. ${ }^{[5}$ In thin films however they must be brought close enough together and be ordered so that there is sufficient overlap of their wavefunctions to facilitate electron transfer. $516121 / 22$

THe conditions which impact the distance are described in the above equation. For processes such as upconversion, $\mathrm{J}$ is very difficult to calculate as the transitions involved in upconversion are forbidden and the spectra barely detectable. ${ }^{622}$ Also the exact shape of the molecular wavefunctions should be known for proper evaluation of $\mathrm{R}$ and $\mathrm{L}$ which is also difficult. 22 Many approximations have been used to deal with these problems, such as the Perrin approximation. ${ }^{22}$ It assumes an active sphere of radius $R_{o}$ for each isolated sensitizer and emitter pair. ${ }^{22} R_{o}$ is termed the Dexter radius. If $\mathrm{L}$ is smaller than or equal to $R_{o}$ it assumes that all energy is totally transferred from sensitizer to emitter. $.6[22$

Thus if the Dexter radius for a given system, one can determine the distance at which upconversion is efficiently occurring. Experimentally the best approach to achieve this is to increase the concentration of sensitizer and emitter to produce optically dense solutions or films. Furthermore in the case of films ensure molecules have long range order to ensure proper overlap of molecular wavefunctions. ${ }^{23}$ 


\subsection{TTA Upconversion in Solution}

The applicability of upconversion is dependent upon being able to harness the process in solid films. Solution phase materials are not desirable for use in conjunction with solar cells due to risk of leakage over time. ${ }^{7}$ But it is by studying the dyes in solution first that the behaviour of the dyes and upconversion as a process can be understood.

Parker and Hatchard were the first to research photon upconversion with polyaromatic dye species. ${ }^{2425}$ It was observed both with a single dye species in solution, and with combinations of dyes that now would be considered as combinations of sensitizers and emitters such as phenanthrene and napthalene. Polyaromatic species have a triplet state that is positioned at around half the energy of the excited singlet state, which encourages self-propagated TTA in solution. It was confirmed as upconversion due to the proportionality of the square of the rate of light absorption which was delayed as a function of the excitation of the sample. ${ }^{24 \mid 26}$ This is because the energy of two photons of the incident light, is emitted as one photon of twice the energy:

$$
I=P_{i n}^{2}
$$

where I is the increase in the generated photocurrent due to upconversion, $P_{i n}$ is the incoming light intensity and 2 is the number of photons required to give rise to the upconverted triplet excited state.

However, as this process was relatively weak in solution, it occurred to Parker and Hatchard that a way of producing these excited triplet states efficiently was needed. They devised different combinations of sensitizers and emitters from polyaromatic hydrocarbons matching their energy require- 
ments to those mentioned above and this improved the process. However, it was not until the 1980's that metallated porphyrins were introduced for use due to their delayed $S_{2}$ fluorescence which improved the process. ${ }^{14}$ This was improved upon again with the utilization of heavy-metal complexes such as palladium octaethylporphyrin. ${ }^{116}$ These sensitizers lead to the efficient production of triplet states in solution owing to the high spin-orbit coupling, resulting in metal-to ligand charge transfer and intersystem crossing ratios of near unity. 19

The recent literature has provided efficient upconversion using various pairs of metallated sensitizers and polyaromatic emitters to span much of the UV-Vis to near-IR region (Fig. 2.4). ${ }^{4223}$

The research has focused on matching different pairs of metallated complexes and emitting molecules to produce the greatest energy gain between the wavelength of absorption of the sensitizer and the emission of the emitter. Energy gains of up to $0.8 \mathrm{eV}$ have been achieved using perylene as the emitter, and platinum (II) tetraphenyltetrabenzoporphyrin as the sensitizer. 27

The two main classes of emitters investigated in this thesis are those based on 9,10-diphenylanthracene (DPA) and 9,10-bis(phenylethynyl)anthracene (BPEA), which have fluorescence quantum efficiencies near unity. $\frac{15128}{12}$ Emitters also have a triplet state that is around half the energy of the singlet state.

As this is a recent area of research, there have been few examples reported of each emitter with various sensitizers. $\frac{115 / 16 / 29 / 30}{1}$ DPA has been used in conjunction with a variety of metallated sensitizers and is one of the most efficient and widely used emitters. ${ }^{4}$ Three common donors have been reported: tris(bipyridine)ruthenium(II) $\left(\left[R u(\text { bpy })_{3}\right]^{2+}\right)$ and its derivatives and platinum octaethyl porphyrin (PtOEP) and palladium octaethyl porphyrin 


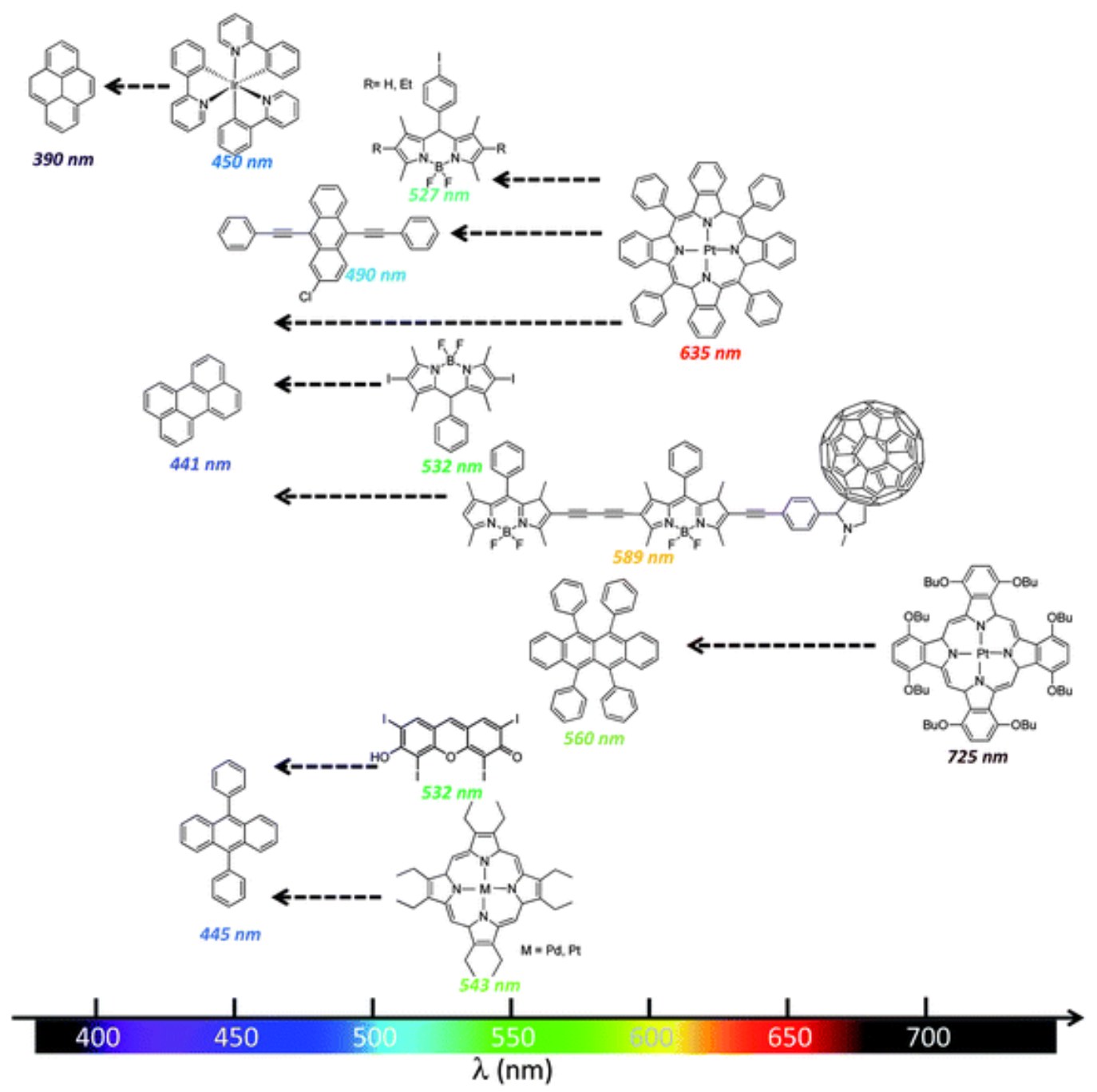

Fig. 2.4 Common sensitizers (right) and emitters (left) used in upconversion. 23 
(PdOEP). 115]18131 These porphyrin species typically have triplet lifetimes on the order of ten's of microseconds (PdOEP is $400 \mu \mathrm{s}$ ) and so serve as energy reservoirs in the upconversion process. $\stackrel{416}{ }$ PdOEP has the best overlap with the triplet state of DPA and gives the highest total energy gain (Table 2.1). 4 However, it should be noted that the Q-band of PdOEP does not have the most efficient overlap with common laser sources typically employed e.g. 532 $\mathrm{nm}$, whereas $\left[R u(\mathrm{bpy})_{3}\right]^{2+}$ and PtOEP have much better overlap, but are less efficient sensitizing agents.

Table 2.1 Excitation and emission wavelengths of common sensitizers used in conjunction with DPA.

\begin{tabular}{ccccc}
\hline \hline Sensitizer & $\lambda_{e x} / \mathrm{nm}$ & $\lambda_{e m} / \mathrm{nm}$ & $\Delta \mathrm{E} / \mathrm{eV}$ & Reference \\
\hline$\left[R u(\text { bpy })_{3}\right]^{2+}$ & 514.5 & 445 & 0.38 & {$[15$} \\
PtOEP & 533 & 440 & 0.49 & {$[31$} \\
PdOEP & 544 & 445 & 0.51 & {$[116$} \\
\hline
\end{tabular}

9,10-Bis(phenylethynyl)anthracene is a molecule which has very high fluorescence efficiency. It has been used in conjunction with sensitizers such as perylene and $\mathrm{C}_{70}$, but not to great effect. ${ }^{32}$ It offers a slightly higher gain in energy than that of the DPA systems. However, it has not been used widely. The main sensitizers with which BPEA has been used are mesotetraphenyl-tetrabenzoporphine palladium $\left(\mathrm{PdPh}_{4} \mathrm{TBP}\right)$ and palladium(II)tetraphenyl-tetrabenzoporphyrin (PdTPBP). Although, there have only been two examples of their use in conjunction with BPEA due to the high cost of the syntheses of these compounds (Table 2.2). ${ }^{16117}$ However, in this research it can be satisfactorily investigated with those sensitizers such as PdOEP or PtOEP whose absorption spectra do not overlap with that of BPEA although it does not offer the maximum energy gain possibly achievable. 
Table 2.2 Excitation and emission wavelengths of common sensitizers used in conjunction with BPEA.

\begin{tabular}{ccccc}
\hline \hline Sensitizer & $\lambda_{e x} / \mathrm{nm}$ & $\lambda_{e m} / \mathrm{nm}$ & $\Delta \mathrm{E} / \mathrm{eV}$ & Reference \\
\hline $\mathrm{PdPh}_{4} \mathrm{TBP}$ & 635 & 490 & 0.58 & $16] 17$ \\
$\mathrm{PdTPBP}^{2}$ & 633 & 515 & 0.45 & 16 \\
\hline
\end{tabular}

\subsubsection{DPA}

There has been much research into anthracene derived systems, and anthracene itself was the beginning of these investigations. However, it has been proven that DPA has a higher singlet fluorescent quantum yield $(\Phi=0.95)$, than that of anthracene $(\Phi=0.1-0.3$, solvent dependent), and accordingly improved upconversion efficiency. 15

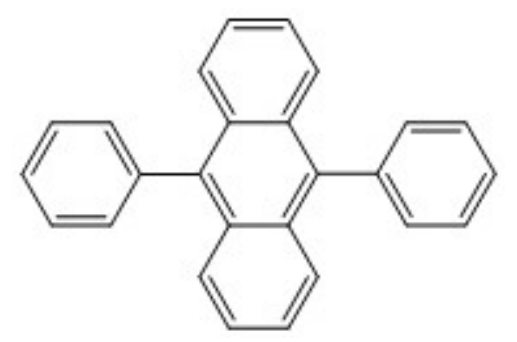

Fig. 2.5 Structure of DPA.

In the infancy of these investigations, anthracene was replaced with DPA in conjunction with $\left[R u(\text { bpy })_{3}\right]^{2+}$ as a sensitizer, which enabled direct visualization of the process at low excitation power $(\lambda=514.5 \mathrm{~nm}, 24 \mathrm{~mW}$ incident power), in conjunction with power dependence measurements which yielded quadratic power dependence as expected. $\frac{15}{15}$

DPA is preferred over anthracene. DPA does not quench the triplet state of the sensitizer at a greater rate as anthracene and DPA have relatively similar quenching constants; anthracene: $K_{s v}=4492 M^{-1}, k_{q}=5.4 \times 10^{9}$ $M^{-1} s^{-1}$ and DPA: $K_{s v}=3465 M^{-1}, k_{q}=4.1 \times 10^{9} M^{-1} s^{-1}\left(K_{s v}=\right.$ Stern- 
Volmer constant, $k_{q}=$ quenching constant). ${ }^{4}$ The same number of triplet states are sensitized in both cases. The increased upconversion efficiency is also not attributed to the increased quantum efficiency of DPA in comparison to anthracene. It is well known that anthracene dimerization is a process that occurs in competition with singlet fluorescence. This pathway becomes deactivated in the case of DPA and BPEA due to the steric hindrance of phenyl and ethynyl substituents at the 9- and 10- positions. It is believed to be this that contributes to the increased efficiency of upconversion. 4115

Work in this field continued towards the enhancement of the process without the presence of a coherent light source (e.g. lasers), increasing feasibility of real-world application. ${ }^{16[29}$ Upconversion is independent of coherence of the excitation light, because it requires resonant single photon absorption. This work employed terrestrial light that was filtered and focused at extralow intensities $c a .10 \mathrm{Wcm}^{-2}$, as opposed to the intensities commonly used on the order of $\mathrm{MWcm}^{-2}$ and $\mathrm{GWcm}^{-2}$. Also, PdOEP was used as a sensitizer, due to the relatively broad absorption spectra and high quantum yields that metal porphyrin (MOEP) based systems offer. This was the first example reported in which noncoherent excited upconversion fluorescence was observed. 29

The dynamics of the process obtained with a $2 \mathrm{wt} \%$ sensitized solution of DPA with PdOEP are shown in Fig. 2.6. This compares the processes occurring; the time evolution of a) upconversion fluorescence of DPA, b) PdOEP fluorescence and c) PdOEP phosphorescence (Fig. 2.6). It was reported that during the first 1 to $2 \mu$ s of the pulse PdOEP phosphorescence occurs, at 662 $\mathrm{nm}$, together with its fluorescence at $549 \mathrm{~nm}$. Starting from the $3 \mathrm{rd} \mu \mathrm{s}$, a strong increase of the upconverted fluorescence is observed, which should be noted is dependent on the intensity of the source. ${ }^{29}$ The rise time is $c a .5 \mu \mathrm{s}$, which is the time necessary for the build up of triplet population states from sensitizer to emitter. It is clear that upconversion is occuring in Fig. 2.6 as 
the decay of the fluorescence is longer compared with the decay of PdOEP phosphorescence.

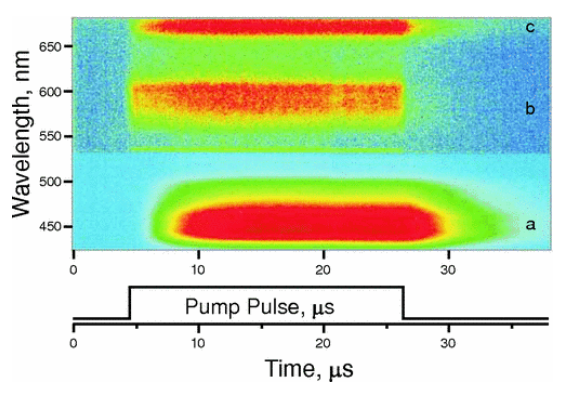

Fig. 2.6 Time evolution of various species imaged with a streak camera: a) upconversion fluorescence of DPA, b) PdOEP fluorescence and c) PdOEP phosphorescence, using an excitation source at $532 \mathrm{~nm}, 80 \mathrm{Wcm}^{-2}$, pulse 23 $\mu \mathrm{s} . \underline{16}$

It was found that upconversion was most efficient at $2 \mathrm{wt} \%$ sensitization using PdOEP. Visual observation of upconversion could be achieved using a solution of this concentration, using the green part of the terrestrial spectrum at an incident intensity of $10 \mathrm{Wcm}^{-2}$. The green terrestrial light was focused to a spot of $1 \mathrm{~mm}$ diameter. An upconversion emission spectrum was obtained, by coupling emitted light into an optical fiber spectrometer. An external efficiency of $1 \%$ was obtained for this process. 29

The variety of sensitizers and emitters investigated has been extended. Ultra-low intensity measurements have been achieved with DPA and PdOEP. Intensities around $50 \mathrm{mWcm}{ }^{-2}$ were used to visually observe upconversion in a solution of concentration DPA $10^{-3} \mathrm{M}$ and PdOEP $10^{-4} \mathrm{M} \cdot 16$ This was done using terrestrial light as an excitation source. The green part of the spectrum was isolated using a dobsonian telescope to collect the light, coupled into an optical fiber which was then filtered to select the green spectral band. This has been the lowest intensity used in the current research field, which suggests that it is becoming more of a possibility that photon upconversion could be harnessed with solar cells. 16 


\subsubsection{BPEA}

Few studies have thus far employed 9,10-bis(phenylethynyl)anthracene (BPEA) as an emitter. It has such a high quantum efficiency that it has been termed the 'light stick' molecule as it glows in daylight (Fig. 2.7). ${ }^{28}$ It has also been used in biological probes as well as in OLED devices due to its high fluorescence quantum yield under terrestrial light. ${ }^{33}$ As an upconversion emitter it requires a sensitizer which would absorb wavelengths lower in the solar spectrum than DPA but the sensitizers required are expensive and not widely available. $4[16|28| 34$

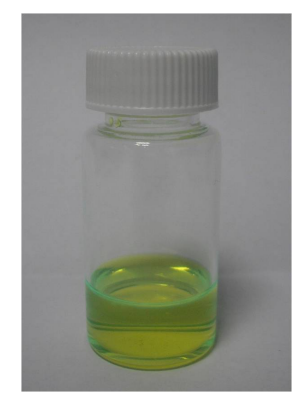

Fig. 2.7 Image of the fluorescence of 9,10-bis(phenylethynyl)anthracene.

Alternative sensitizers has been investigated, that are not heavy metal based. One such example uses $\mathrm{C}_{70}$, which has a broad spectral absorption, centering at $470 \mathrm{~nm}$ with a high ISC quantum yield ( $c a .1) .[32$ No deoxygenation of solutions was required, but such systems produce a quantum external efficiency of $0.1 \%$ which is lower than the $3.2 \%$ obtained with the sensitizer meso-tetraphenyl-tetrabenzoporphyrin palladium $\left(\mathrm{PdPh}_{4} \mathrm{TBP}\right) .16[32$

Therefore, studies have focused on using BPEA in conjunction with $\mathrm{PdPh}_{4} \mathrm{TBP}$ and PdTPBP as sensitizers. Streak camera images using a coherent light source have shown that the rise-time of upconverted fluorescence for $\mathrm{PdPh}_{4} \mathrm{TBP}$ and BPEA pair varied between $8 \mu$ s and $30 \mu \mathrm{s}$, depending on the intensity of excitation light. Upconversion was visually observed in the same solu- 


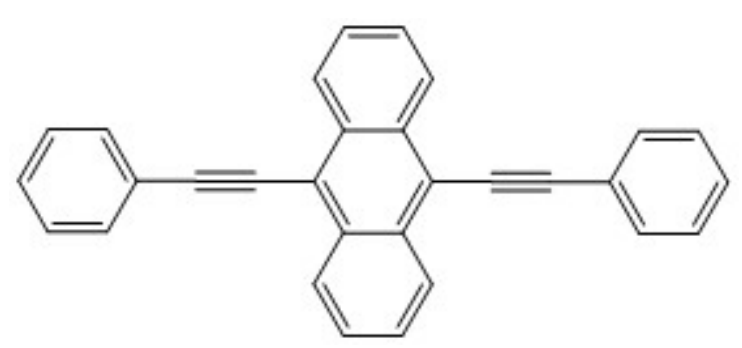

Fig. 2.8 Schematic of BPEA.

tion, using an excitation wavelength of $635 \mathrm{~nm}$ at ultra-low intensity of 70 $\mathrm{mWcm} \mathrm{cm}^{-2}$ and a spot size exceeding $0.1 \mathrm{~cm}^{-2}$. The highest upconversion external quantum efficiency for this system was $3.2 \%$ for a solution where the concentrations of the species were $2 \times 10^{-5} \mathrm{M}$ sensitizer and $2 \times 10^{-4} \mathrm{M}$ emitter. ${ }^{16}$ In a separate measurement sunlight has also been used as an excitation source centered at $630 \mathrm{~nm}$. Emission spectra were obtained at an intensity of $1 \mathrm{Wcm}^{-2}$, which is the first reported instance of sunlight as an excitation source for this upconversion pair. $\frac{16}{16}$ This is an achievement as the harnessing of upconversion at low intensities furthers the possibility of being able to use it in conjunction with the sun, which also has intensities within this region.

Although not ideal, experiments below with BPEA will be used in conjunction with sensitizers such as PdOEP. They do not offer the highest energy gain but they can be used to prove that TTA, the key step in upconversion is occurring.

\subsection{TTA Upconversion in Solids}

Upconversion in solid films, though more desirable for photovoltaic and technological applications, provides certain challenges. The process in solution requires efficient diffusion of molecules, but in solid films this is no longer 
possible. Sensitizers and emitters will not move freely, molecules must be brought within the range on which Dexter transfer occurs (10 $\AA)$ and exhibit long range order to ensure the proper overlap of molecular wavefunctions. $\frac{[56}{56}$

The majority of materials previously investigated for solid state applications have relied on low glass transition temperatures and low crystallinity, which allow for translational and rotational mobility for either chromophore or exciton diffusion. These materials only decrease the efficiency of the process compared with solutions because diffusion in rubber-like materials becomes much slower. 127

The first example of upconversion in thin films was performed by the Castellano and Weder groups. 1 This involved blending PdOEP and DPA into a matrix consisting of a copolymer of ethyleneoxide/epichlorohydrin which was photophysically inert, amongst having other desirable properties. 1 Its glass transition temperature is $236 \mathrm{~K}$, so that at room temperature it is in a rubbery state allowing sufficient translational and rotational mobility for the embedded molecules.

Upconversion was evidenced within blended films of PdOEP and DPA by the quenching observed of the triplet state, and the evolving of the delayed fluorescence emitted from DPA in time-resolved transient absorption. ${ }^{10}$ It did not provide an increase in efficiency from solution upconversion but it was thought that the films offered greater protection from quenching of triplet states by oxygen. 23

Studies were furthered on the effect of temperature, where the polymer host was varied to represent different glass transition temperatures. This enabled increased diffusion of molecules through the material, and thus the upconversion efficiency increased. ${ }^{23127}$ However, the efficiency was still worse than that observed in liquid, and it is not a true solid film material. 
There is one instance of BPEA being used in solid films, where BPEA and $\mathrm{PdPh}_{4} \mathrm{TBP}$ were blended into films of cellulose acetate. Photoluminescence spectra were obtained for the fluorescence upconversion emitted from these films at ultra low incident power densities $c a .1 \mathrm{mWcm}^{-2}$ or $3.2 \times 10^{15}$ photons $s^{-1} \mathrm{~cm}^{-2}$. They concluded that the upconversion efficiency was still three orders of magnitude lower than in solution. ${ }^{17}$ The work performed thus far on utilising PdOEP, BPA and BPEA in solid films has been limited to low - glass transition temperature molecules, where molecules are limited by diffusion.

Liquid crystalline derivatives or room temperature neat liquids of derivatives of common upconversion emitters would offer an opportunity to control the electronic coupling between these molecules and bring them within the optimal length and time scales (Fig. 2.9). In the liquid crystalline phase, the way in which they arrange with respect to each other can be controlled by controlling the phase. The creation of a liquid crystalline material that may facilitate upconversion has to my knowledge never been attempted before. 


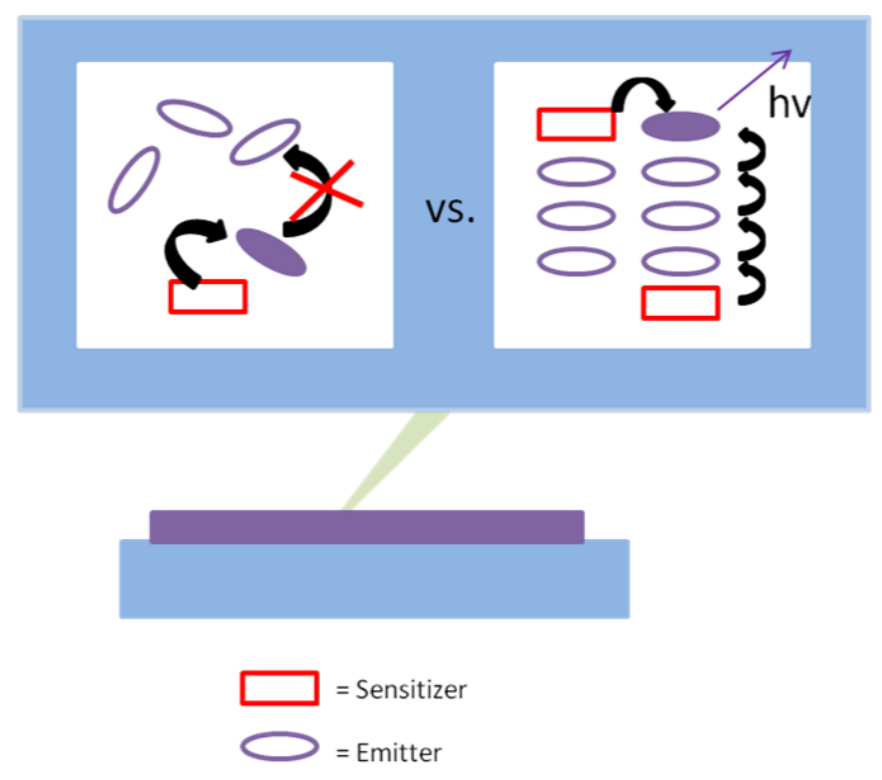

Fig. 2.9 Schematic of the effect of liquid crystals on exciton diffusion in solid films. 


\section{Chapter 3}

\section{Liquid Crystals}

Liquid crystals are an intermediate phase of matter between that of a solid and a liquid. They exhibit long range order similar to that of a crystalline solid, but retain the flow properties of a liquid. $35[36$

This order means that materials are highly anisotropic, but also that they can enhance many processes such as charge mobility. ${ }^{37}$ As discussed previously, liquid crystalline derivatives of common upconversion emitters would offer an opportunity to control the electronic coupling between these molecules and bring them within the optimal length and time scales required. It is therefore important to understand the relationship between the structure of the molecule and the mesomorphic behaviour. 


\subsection{Background}

The discovery of liquid crystals has largely been attributed to Reinitzer, in his investigation of cholesteryl benzoate in $1888 .{ }^{38}$ He found that the material formed a cloudy liquid before melting completely. Liquid crystals are currently best known for their use as liquid crystalline displays or LCDs. $\underline{36 / 39 / 40}$ The way in which these molecules self-assemble classifies the phase formed. The type of phase formed can be controlled by the shape and chemical functionality of the molecule.

Molecules have two main requirements to be liquid crystalline: they must have a rigid core and long flexible alkyl chains. $\frac{35 \mid 36 / 39411}{13}$ The rigid core induces structural order within the phase to align the molecules in a certain direction. The long and flexible alkyl chains provide sufficient entropy to overcome the enthalpy of crystallisation, and therefore provide fluidity to the phase. 35 The entropy force prevents crystallisation and allows for formation of the mesophase. This occurs at a specific temperature or temperatures intermediate between the initial melting point, and the temperature at which a normal isotropic liquid is formed. The core length and shape, tail length and positioning determine whether liquid crystalline properties are observed or not. $\underline{40}$

The transition between these phases can be induced by altering a variety of external and internal properties: temperature, concentration, light, electric field or mechanical shear. $\frac{36[40}{}$ Liquid crystals whose mesomorphic phases are destroyed by temperature or a change in concentration are termed thermotropic and lyotropic respectively. ${ }^{41}$ However, thermotropic liquid crystals will be the only type considered.

The formation of thermotropic liquid crystalline phases depends on the 
molecules having sufficient structural rigidity to impart some order but enough conformational freedom to impart disorder. ${ }^{42}$ This has been known for a long time. ${ }^{40} \sqrt[42]{4}$ There are classes of molecules that have a greater tendency to form liquid crystals than others (Scheme 3.1). 42

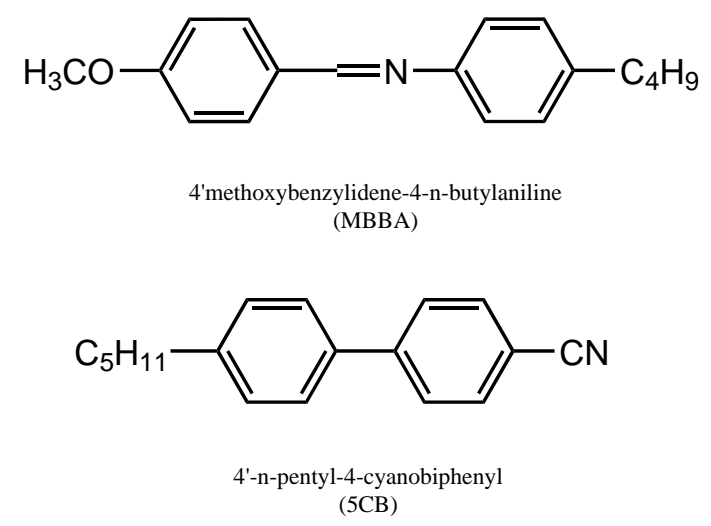

Scheme 3.1 Lath-like molecules that exhibit the formation of liquid crystal phases.

Many liquid crystal molecules have a common geometrical feature that they are markedly elongated or 'rod-like'. Examples of this are 4'-methoxybenzylidene4-n-butylaniline (MBBA) and 4'-n-phenyl-4-cyanobiphenyl (5CB). $\stackrel{42}{\text { Their }}$ elongated lath-like core encourages the molecules to lie a long their long axis. Furthering this, many molecules which appear to have long elongated structures when drawn in a particular confirmation may not retain this shape in the fluidity of the liquid phase. $\stackrel{42}{2}$ They may assume non-linear confirmations by rotations around bonds. Therefore rigidity is an essential component of the molecule. This means stillbenes (Scheme 3.2) which contain double bonds have a greater tendency to form liquid crystals than their single bonded 1,2-diphenylethane counterparts. ${ }^{42}$

As a consequence, the majority of liquid crystals contain aromatic rings. 

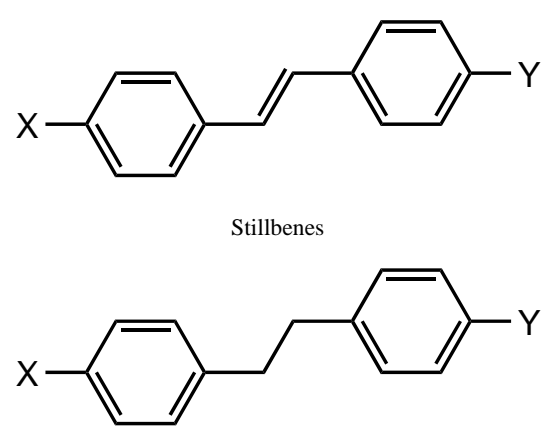

1,2-diphenylethanes

Scheme 3.2 Liquid crystallinity in double bonded vs. single bonded molecules in which $\mathrm{X}$ and $\mathrm{Y}$ represent various alkyl chain substituents.

Apart from providing rigidity they also contain $s p^{2}$ hybridised carbons which provide polarisability to the system. $\underline{42}$

Despite all this, it is impossible to design a molecule and know it will be a liquid crystal. For the purpose of this research liquid crystals had to encompass the cores of common upconversion emitters: 9,10-diphenylanthracene and 9,10-bis(phenylethynyl)anthracene. Thus, the literature was first investigated for similar structures that have exhibited liquid crystallinity. The target molecules in chapters 4 and 5 were designed with this in mind, but also to resemble long lath-shaped molecules which was achieved by the addition of long alkyl chains.

\subsection{Liquid Crystal Phases}

The phases formed are defined by the shape of the mesogen (the molecule that exhibits liquid crystalline behaviour), which can be either rod shaped i.e. 'calamitic' or disc shaped i.e. 'discotic'. 40 The shape is defined by the 
type of rigid core, and the number of long alkyl tails attached to the central core of the molecule. $\underline{40}$

The molecules exhibit different degrees of orientational and positional order depending on the type of phase formed. $\stackrel{40}{ }$ The type of phase formed can be detected experimentally by polarized optical microscopy (POM), whereby the material is placed between two crossed polarizing plates and the resulting pattern of light, which is characteristic of each liquid crystalline phase, is observed. $\underline{4143}$

\subsubsection{Calamitic Mesogens}

Calamitic mesogens form either nematic or smectic mesophases. The nematic phase is the simplest phase containing one degree of ordering, in that molecules are disordered with respect to each other, but lie along the molecular axis point in the same direction defined by a director (n) Fig. 3.1). 41

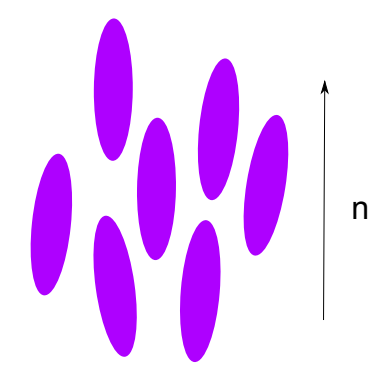

Fig. 3.1 Calamitic mesogens aligned in a nematic phase.

This phase has low viscosity and more closely resembles the flow properties of a liquid. 40

The second type of phase formed by calamitic mesogens is a smectic phase, which is more ordered and occurs at lower temperatures compared to that of 
the nematic phase. It is polymorphic in that there are many different phase types that can be observed, which are defined by the letter following the phase e.g. A, B or C, $\mathrm{C}^{*} . \underline{40141}$ In this phase, mesogens have both directional and positional order. They arrange themselves according to a director on the long axis, and are also arranged in layers. $\frac{40141}{1 n}$ the smectic A phase the director is aligned with the normal of the layers. However, in a smectic $\mathrm{C}$ phase the director is tilted with respect to the normal of the layer planes Fig. 3.2. 39140

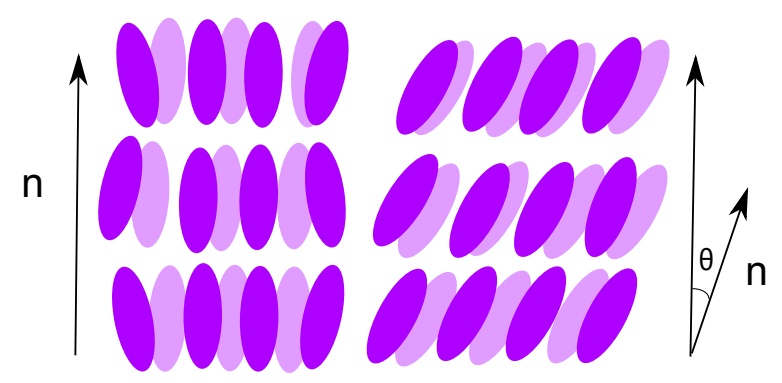

Fig. 3.2 Calamitic mesogens aligned in smectic A (left) and smectic C mesophases (right).

\subsubsection{Discotic Mesogens}

The second broad class of mesogen is discotic, which are known to form nematic and columnar phases. ${ }^{40141}$ In discotic mesogens the director vector is a normal vector to the plane of the disc. The ordering in the nematic phase is the same as for calamitic mesogens, although they are considered distinctly different phases. $\underline{40}$

The other type of discotic phase which can be formed is a columnar phase where the disc shaped molecules self-assemble in columns (Fig. 3.3). This phase has two degrees of order as well as orientational order and is 

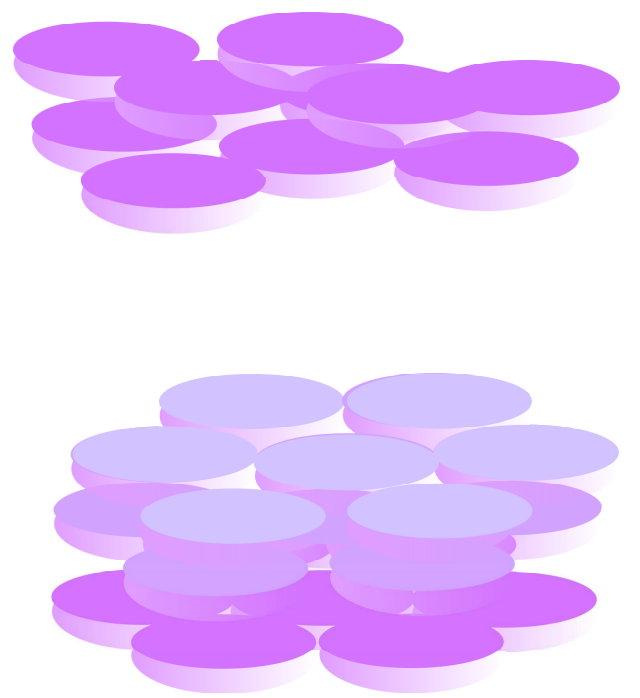

Fig. 3.3 Discotic mesogens aligned in a nematic (top) and columnar phase (bottom).

more ordered than the calamitic smectic phase. The columnar mesophases are classed according to the type of packing they exhibit e.g. hexagonal, rectangular and oblique mesophases. 40

The techniques used to investigate liquid crystalline phases are differential scanning calorimetry (DSC) and polarised optical microscopy (POM). Each technique used in isolation may not give a complete understanding of the behaviour of the system; POM provides information of which phases are formed, and DSC provides precise temperatures and enthalpies of the transitions, thus the two must be used in conjunction with each other so as to completely describe the behaviour of the phase. DSC ascertains at what temperatures phase changes occur, and POM exploits the defects and birefringence of the liquid crystal textures in order to assign the phase. Both techniques DSC and POM were used in conjunction to investigate the behaviour of the compounds, and were used in this way with all compounds synthesized in this thesis. Information on these techniques and how they were used can be found in the experimental section. 


\subsection{Anthracene Derived Liquid Crystals}

The majority of common upconversion emitter cores, as discussed in chapter 2 , have been derivatives of anthracene. ${ }^{4}$ In order to synthesize liquid crystalline derivatives of anthracene, the requirements for such molecules to exhibit such behaviour must be examined.

A variety of anthracene derived liquid crystals have been reported in the literature. 44 The central polyaromatic core has been chosen despite its elongation, for its efficient photophysical properties. ${ }^{37}$ It has also been exploited in a variety of technological applications such as a light emitting layer for organic light-emitting diode (OLED) devices and as a laser dye for liquid-crystal-lasers. $\frac{944448}{14}$ The liquid crystalline phases exhibited have imparted interesting properties; the close proximity of the molecules causes the $\pi$ orbitals to overlap, giving rise to high charge-carrier mobilities and enhanced conduction which also would be favourable in an upconversion application. $\stackrel{37}{ }$

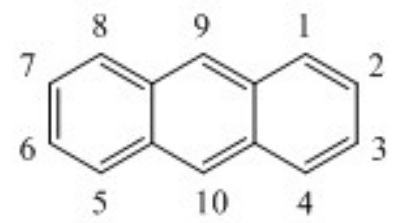

Fig. 3.4 Substitution positions of anthracene.

There are two main classes of substitution at the $9,10^{8 / 9 / 44}$ and $2,6^{37 / 49}$ positions of the anthracene ring (Fig. 3.4). There have been a variety of attachments and substituents including different length alkyl chains, phenylsubstituents or phenylethynyl substitutents with chains attached at the 4 position.

The following discussion will focus solely on 9,10-diphenylanthracene and 9,10-bis (phenylethynyl)anthracene which are both upconversion emitting 
cores studied in this thesis.

\section{$3.49,10-S u b s t i t u t i o n$}

Substitution at the 9,10-positions of anthracene have been performed to produce liquid crystalline derivatives of common dye species. $\frac{8|9| 44 \mid 50}{9,10-}$ diphenylanthracene (DPA) and 9,10-bis(phenylethynyl)anthracene (BPEA) exhibit high fluorescence quantum yields and the transition dipole moment of the longest wavelength absorption is polarized parallel to the long axis in these molecules, which gives rise to directionally dependent photophysical effects. ${ }^{9|44| 48}$ The majority of these 9,10-substituted liquid crystals have mesophase temperatures in the range of $100-250^{\circ} \mathrm{C} .819144$ The polyaromatic core forms the rigid central core of the liquid crystal, which must be compensated for by the use of long flexible chains to allow formation of a liquid crystalline phase.

\subsubsection{DPA Derivatives}

DPA is an extremely efficient emitter used in upconversion. $\frac{4}{4}$ This molecule has a rigid core which is favourable in mesophase formation, and retains pure blue fluorescence $(440 \mathrm{~nm}) \cdot{ }^{9 \sqrt{44}}$ However, the central molecule does not sit planar. Hydrogen repulsion between the hydrogen atoms at the 2- and 6- positions on the phenyl rings and the 1-,4-,5- and 8- positions means that the outer phenyl rings sit perpendicular to the central core. ${ }^{9[44}$ Although this prevents anthracene dimerization, it may disrupt close packing of the molecules in a mesophase. 
The area of liquid crystals based on 9,10-diphenylanthracene has not been explored to a great extent previously, and as a consequence there have not been a great number of liquid crystalline derivatives of the DPA core reported. However, for upconversion it would be highly advantageous to synthesize a liquid crystalline derivative based on 9,10-diphenylanthracene (1) Scheme 3.3.

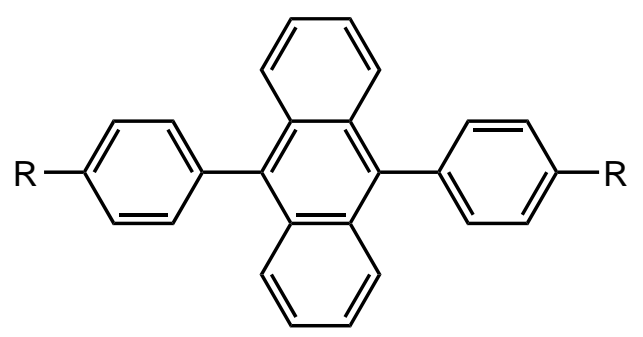

1

$\mathrm{R}-\mathrm{OC}_{6} \mathrm{H}_{12} \mathrm{OH}$

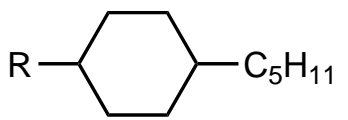

1a

1b

Scheme 3.3 Liquid crystalline derivatives of DPA.

One liquid crystal reported was 9,10-bis(4-(6-hydroxyhexyloxy)phenyl)anthracene (1a), synthesized for use in liquid crystal lasing devices. ${ }^{44}$ This molecule was incorporated into the polymer main chain of a chiral polyester copolymer by covalent linkage through the hydroxyl groups present at the end of the hexyl chains. ${ }^{44}$ It should be noted that this polymer, BB-4*/6(70/30) does exhibit liquid crystalline behaviour, it has phase transitions of the following order: [crystal-Sm C*-Sm A-isotropic]. ${ }^{44}$ The liquid crystalline behaviour was further investigated by variation of the concentration of the diphenylanthracene dye compared with the copolymer from 1 - $10 \mathrm{~mol} \%$. The increasing presence 
of the dye decreased the transition temperatures of the phases observed in each polymer. $\stackrel{44}{4}$

At low concentration, similar phase transitions to the polyester were observed, where on cooling a smectic A phase, with a fan-like texture was observed at $220^{\circ} \mathrm{C}$, followed by the formation of a broken fan-like texture indicative of smectic $\mathrm{C}^{*}$ phase. ${ }^{44}$ With increasing dye concentration (5 and 10 mol\%), it was found that only the smectic A phase was observed forming at $160^{\circ} \mathrm{C}$, while the smectic $\mathrm{C}^{*}$ phase was lost. ${ }^{44}$ It should be noted that most of the liquid crystalline behaviour observed was imparted by the polymer itself in this example.

A second liquid crystal was synthesized by the extension of the central diphenylanthracene core by addition of cyclohexyl rings with pentyl chains attached at either end (1b). ${ }^{9}$ This compound 9,10-bis(4-(4-pentylcyclohexyl) phenyl)anthracene or BPCPA was synthesized for use as a material for the light emitting layer in OLED devices. ${ }^{9} \mathrm{~A}$ mesophase was observed at $276^{\circ} \mathrm{C}$, where a smectic A phase was formed, with a mosaic like texture. $\frac{9}{9}$ This had a much higher mesophasic temperature than the previous liquid crystal example, as the core has been extended by a cyclohexane ring.

It was thought, based on these examples that the requirements for liquid crystalline behaviour were extension of the central core at the $\mathrm{R}$ positions with alkyl chains longer than $n=6$, or for additional alkyl chains to be added at the 3 and 5 positions on the outer phenyl ring. This trialkyloxy moiety is a common motif used in synthesis to induce liquid crystalline behaviour, where more alkyl chains are likely to induce increased entropy to the system to overcome the enthalpy of crystallisation provided by the rigid core. $[51$ 


\subsubsection{BPEA Derivatives}

BPEA is another popular fluorescent dye molecule and upconversion emitter (2). It is so fluorescent that it has been termed a 'light-stick' compound due to its bright fluorescence observed under ambient light. ${ }^{34152}$ The main advantage over DPA is that the triple bonds present at either end of the molecule allow free-rotation of the rings leading to the possibility of a more planar core. $\underline{8[50}$

BPEA itself does not exhibit liquid crystalline behaviour, nor do any derivatives that contain small substituents. ${ }^{53154}$ This has been attributed to the low length to breadth ratio, as the broad core disrupts the rod-like profile of analogous tolanes for which smectic and nematic phases have formed Fig. 3.5. 55 A variety of substituents were studied in their attachment to the BPEA core at the $\mathrm{R}$ and $R^{\prime}$ positions (Scheme 3.4). $\frac{8150}{}$ The majority of molecules that were mesomorphic, were either not symmetrical with different length chains in the $\mathrm{R}$ and $R^{\prime}$ positions, or were extended out with a phenyl ring which had a pentyl chain attached to the 4 position. 8

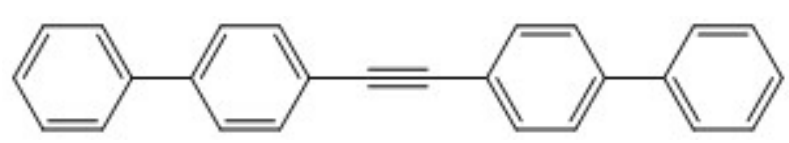

Fig. 3.5 Generic tolane structure.

One such involved the addition of a decyl carboxylic acid chain to one side of the PEA core, with a ethyloxy substituent on the other $(\mathbf{2 a}) . \sqrt[8]{ }$ This compound melted at $125^{\circ} \mathrm{C}$ on heating and a nematic liquid crystalline phase formed at $117^{\circ} \mathrm{C}$ on cooling. However, on symmetrisation, where the same decyl carboxylic chain was attached at both $\mathrm{R}$ and $R^{\prime}$ positions $(\mathbf{2} \mathbf{b})$, no liquid crystalline behaviour was observed. ${ }^{8}$ However, it is interesting to note that these simplest compounds $(\mathbf{2 a}, \mathbf{2} \mathbf{b})$ exhibit polymorphism on crystallisation. 


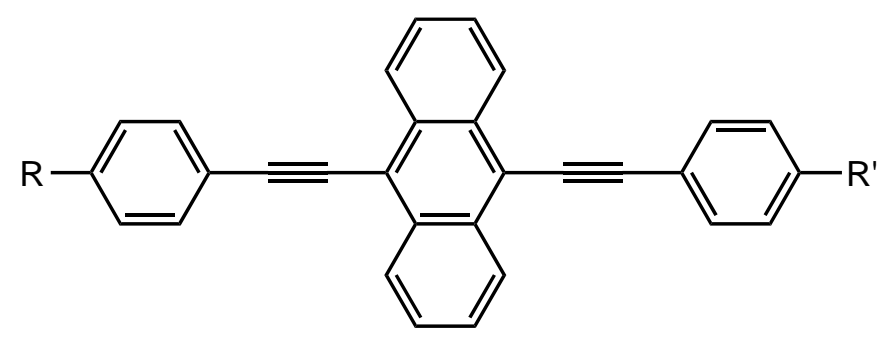

2

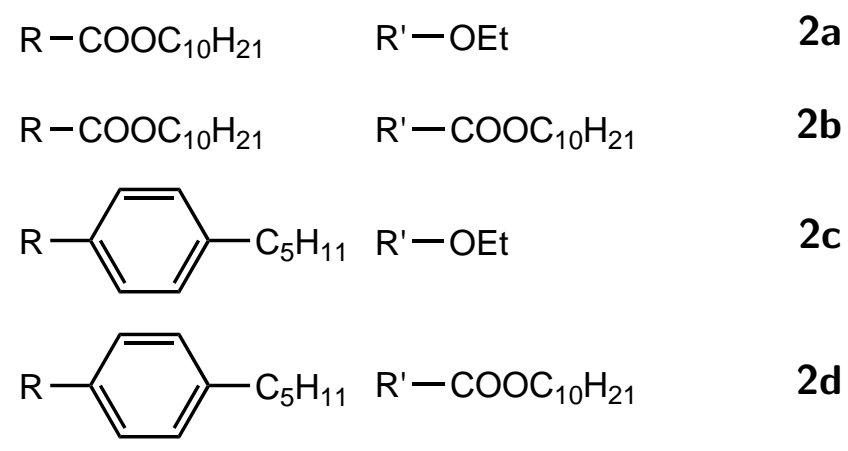

Scheme 3.4 Liquid crystalline derivatives of BPEA.

For $(\mathbf{2 a})$, two crystalline variants were formed at $110^{\circ} \mathrm{C}$, one was an orange solid, and the second was a dark red solid. On reheating, these variants had different melting points at 123 and $131^{\circ} \mathrm{C}$ respectively. In the case of (2b), low temperature red crystals transformed on heating at $104{ }^{\circ} \mathrm{C}$ into yelllow crystals before melting at $132^{\circ} \mathrm{C} . \underline{8}$ On cooling, the yellow crystalline solid was formed directly at $119{ }^{\circ} \mathrm{C}$, which over time reverted back to the more thermodynamically stable red solid. ${ }^{8}$

Extension of the central core with phenyl rings at the $\mathrm{R}$ and $R^{\prime}$ positions, induced the formation of nematic liquid crystalline phases. The liquid crystalline phases observed also became enantiotropic. This means the same phases were formed on both heating and cooling of the sample which is not typical of liquid crystalline materials which often form different phases on heating and cooling. ${ }^{8}$ This was expected, where the addition of aromatic 
rings favoured the intermolecular interactions across the long axis, which reduced the effect of the broad anthracene core at the centre as well as more fully representing the tolanes which are known to exhibit both nematic and smectic phases. $\frac{55}{5}$

With the first substitution, in the addition of an aromatic 4-pentylphenyl group to one side of the core, with the retention of the ethoxy substituent at $R^{\prime}$ (2c), a nematic phase was observed to form at $191^{\circ} \mathrm{C}$, which was directly followed by decomposition at $260^{\circ} \mathrm{C} .{ }^{8}$ However, when the ethoxy substituent at $\mathrm{R}$ ' was replaced with a decyl carboxyl chain $(\mathbf{2 d})$, polymorphism was observed where yellow crystals at room temperature transformed into orange crystals at $130{ }^{\circ} \mathrm{C}$, followed by a nematic phase at $147^{\circ} \mathrm{C}$ and the isotropic phase at $209^{\circ} \mathrm{C} .{ }^{8} \mathrm{On}$ cooling, a nematic phase was formed at $208^{\circ} \mathrm{C}$, followed by formation of orange crystals at $125^{\circ} \mathrm{C}$ and yellow crystals at $102^{\circ} \mathrm{C}$. 8

A new core, 9,10-bis(biphenyl-4-ylethynyl)anthracene (BBPEA) has been investigated Scheme 3.5, .8150 The derivatives of the BBPEA core which exhibited liquid crystalline behaviour were the molecules where alkyl or alkylether chains had been attached. Compound (3a) exhibited the formation of a nematic phase at $241^{\circ} \mathrm{C}$ which was followed by decomposition at $270{ }^{\circ} \mathrm{C} .8$ The use of alkylether chains brought the transition temperatures down significantly. Compound (3b) exhibited the formation of a nematic phase at $56.1^{\circ} \mathrm{C}$ before the formation of the isotropic phase at $132.2^{\circ} \mathrm{C} .50$ On cooling, a Schlieren texture was observed, followed by formation of a fan shaped conical nematic phase. ${ }^{50}$ The extension of the central core by phenylethynyl substituents as in compounds (3c) and (3d) did not lead to formation of liquid crystalline phases. This was attributed to the increased driving force of $\pi-\pi$ stacking introduced by the extension of the polyaromatic core leading to crystallinity ${ }^{50}$ Compound (3e) exhibited similar phases to (3b). A nematic phase was formed at $95.9^{\circ} \mathrm{C}$ which was followed by the formation of the isotropic liquid at $146.6^{\circ} \mathrm{C}$. Upon cooling again a fan tex- 
ture was observed indicating formation of a columnar phase, but the nematic phase was not observed. $\underline{50}$

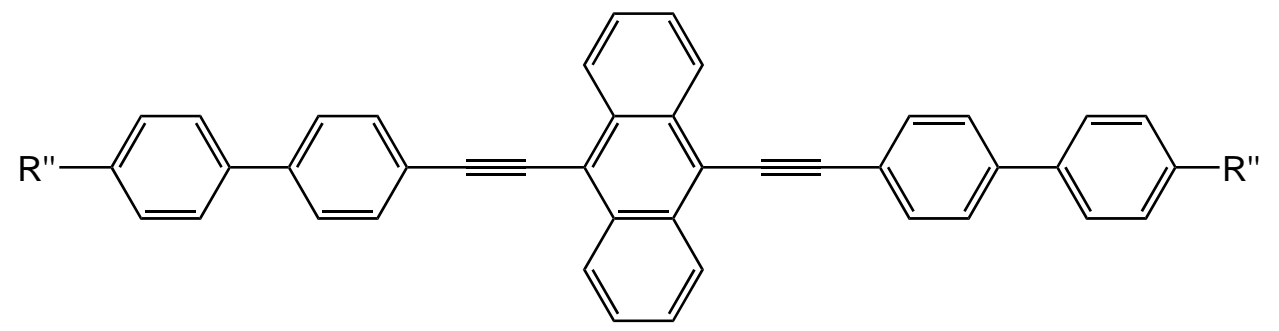

3

$\mathrm{R} "-\mathrm{OC}_{5} \mathrm{H}_{11}$

$\mathrm{R} "-\mathrm{O}\left(\mathrm{CH}_{2} \mathrm{CH}_{2} \mathrm{O}\right)_{5} \mathrm{CH}_{3}$
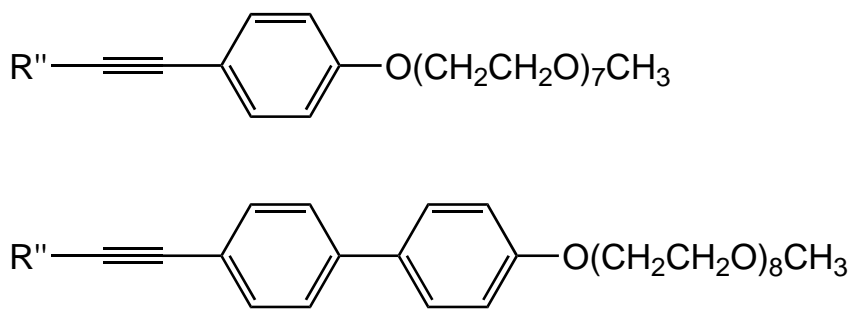

$\mathrm{R} "-\mathrm{O}\left(\mathrm{CH}_{2} \mathrm{CH}_{2} \mathrm{O}\right)_{3} \mathrm{CH}_{3}$ 3a

$3 b$

$3 c$

$3 d$

$3 e$

Scheme 3.5 Liquid crystalline derivatives of BBPEA.

It was thought based on these examples, the requirements for liquid crystalline behaviour of BPEA derivatives was extension of the central core with akyl chains with or without a phenyl linker. The other possibility was to synthesize asymmetric derivatives of BPEA where different length alkyl chains were attached, however its synthesis was not efficient as it relied on the production of statistical mixtures. The syntheses of derivatives based on DPA 
and BPEA in the following chapters were based on the compounds which exhibited liquid crystalline behaviour summarised in this chapter. 


\section{Chapter 4}

\section{9,10-Diphenylanthracene (DPA) Derivatives}

Five derivatives of 9,10-diphenylanthracene (DPA) were synthesized in which various alkyl chains were attached to the central core. DPA does not exhibit mesomorphic behaviour itself, so in order to overcome the enthalpy of crystallisation of the rigid central core, long flexible alkyl chains were attached which provide entropy to the system. The term 'catenar' will be used to refer to the number of long alkyl chains attached to the central core. Three dicatenar derivatives were synthesized, and the attached alkyl chain varied in length; tails of 8, 12 and 16 carbons were used. Two entirely novel hexacatenar derivatives were synthesized, and the attached alkyl chain varied again; tails of 10 and 16 carbons were used. The attachment of alkyl chains directly to the central core in contrast to extension of the central core by further aromatic rings provided the most direct approach to induce liquid crystalline behaviour without altering the photophysical properties of the DPA dye. ${ }^{9144}$

In this chapter synthetic routes for each derivative will be detailed, as 
well as the phase behaviour for each derivative, though the full experimental and characterisation information will not be discussed. Full synthetic and experimental procedures and compound characterisation can be found in the experimental chapter.

\subsection{Synthesis of Dicatenar DPA Derivatives}

The synthesis involved four steps Scheme 4.1). The reagent 4-bromoanisole was reacted with magnesium in order to form a Grignard reagent under inert conditions, which was then reacted with trimethylborate to form 4methoxyphenyl boronic acid (4).

A Suzuki coupling of (4) and 9,10-dibromoanthracene (5) under inert conditions was carried out to obtain dianisylanthracene (6) close to $100 \%$ yield. A particularly bulky phosphine was needed in conjunction with such bulky substituents to achieve such a good yield. Tri-tert-butylphosphonium tetrafluoroborate was used, in conjunction with tris(dibenzylidenecacetone) dipalladium(0). Reactions between similar bulky substituents have been previously achieved with good yield in the literature. $\underline{56}+58$

The next step involved a deprotection of the outer methoxy groups. The most common reagent for which this is achieved effectively is hydrobromic acid. ${ }^{44}$ An extremely hygroscopic green solid of 9,10-bis(4-(hydroxy)phenyl) anthracene (7) was obtained, which was used directly in the final Williamson ether syntheses without further purification. The final compounds; 9,10bis(4-(octyloxy)phenyl)anthracene (8), 9,10-bis(4-(dodecyloxy) phenyl) anthracene (9) and 9,10-bis(4-(hexadecyloxy)phenyl)anthracene (10), were obtained in good yield and purified by recrystallisation. 
<smiles>COc1ccc(-c2c3ccccc3c(-c3ccc(OC)cc3)c3ccccc23)cc1</smiles>

$(98 \%)$

4<smiles></smiles>

$(99 \%)$

7<smiles>CCCOc1ccc(-c2c3ccccc3c(-c3ccc(O)cc3)c3ccccc23)cc1</smiles>

$$
\begin{array}{cl}
\mathbf{8} & \mathrm{R}=\mathrm{C}_{8} \mathrm{H}_{17}(42 \%) \\
\mathbf{9} & \mathrm{R}=\mathrm{C}_{12} \mathrm{H}_{25}(33 \%) \\
\mathbf{1 0} & \mathrm{R}=\mathrm{C}_{16} \mathrm{H}_{33}(21 \%)
\end{array}
$$

Scheme 4.1 Synthesis of dicatenar derivatives of DPA. 


\subsection{Phase Behaviour of Dicatenar DPA Deriva- tives}

The synthesized derivatives $(\mathbf{8}),(\mathbf{9})$ and (10) were investigated for the presence of mesomorphic behaviour using polarised optical microscopy and by examining the traces obtained by differential scanning calorimetry (DSC).

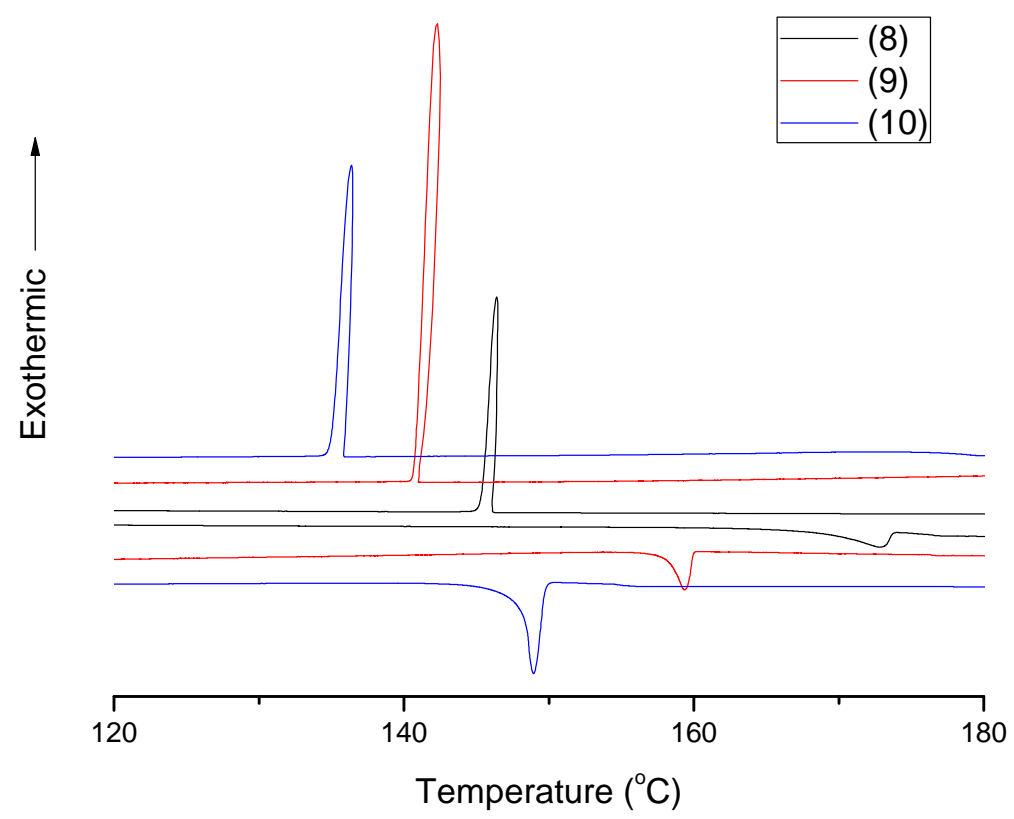

Fig. 4.1 DSC plot for dicatenar DPA derivatives, $(\mathbf{8})$ and $(\mathbf{1 0})$ at a rate of $1^{\circ} \mathrm{C} \mathrm{min}^{-1}$ and $(\mathbf{9})$ at a rate of $0.5^{\circ} \mathrm{C} \mathrm{min}^{-1}$.

On heating and cooling it was observed in the DSC trace there was only one peak observed for each compound (Fig. 4.1). On cycling and repetition of these measurements the same behaviour was observed. The melting and crystallisation points for these derivatives occur between 135 and $170{ }^{\circ} \mathrm{C}(\mathrm{Ta}-$ 
ble 4.1). DPA itself has a melting point of $248^{\circ} \mathrm{C}$, with the addition of octyl chains, this shifts to $169.65^{\circ} \mathrm{C}$ as entropy is increased by the addition of long flexible alkyl chains. This trend is continued as the attached alkyl chains are increased in length, the melting and crystallisation points decrease further in temperature.

Table 4.1 Melting and crystallisation temperatures and their associated enthalpies for dicatenar DPA derivatives.

\begin{tabular}{cccc}
\hline \hline Compound & Transition & Temperature ${ }^{\circ} \mathrm{C}$ & Heat $/ \mathrm{Jg}^{-1}$ \\
\hline$(\mathbf{8})$ & Melting & 169.65 & -29.09 \\
- & Crystallisation & 146.10 & 68.66 \\
$(\mathbf{9})$ & Melting & 158.25 & -75.48 \\
- & Crystallisation & 141.07 & 88.56 \\
$(\mathbf{1 0})$ & Melting & 147.88 & -87.84 \\
- & Crystallisation & 135.92 & 97.13 \\
\hline
\end{tabular}

The DSC trace alone cannot indicate the presence of a liquid crystalline phase, but can show only at what temperatures transitions do occur within the material. Thus examination under the polarised optical microscope was needed to determine if the derivatives were liquid crystals.
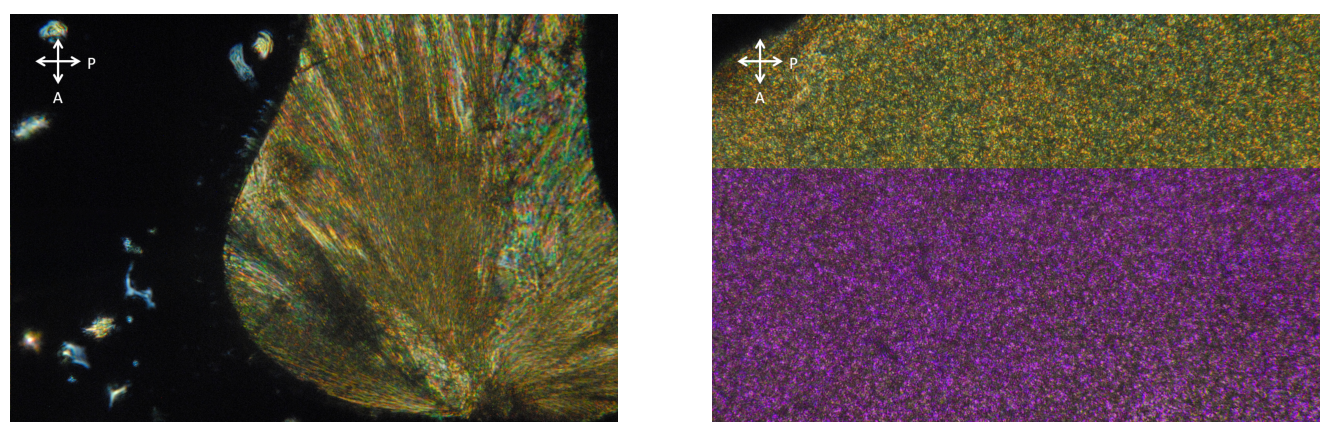

Fig. 4.2 POM images of (9) (left) at $157.4^{\circ} \mathrm{C}$ at $100 \mathrm{x}$ magnification and (10) (right) at $147.9^{\circ} \mathrm{C}$ at $200 \mathrm{x}$ magnification.

All compounds consisted of a gold crystalline solid up until melting (Fig. 4.2). The crystalline solid of (10) was the most birefringent (Fig. 4.3). In each 

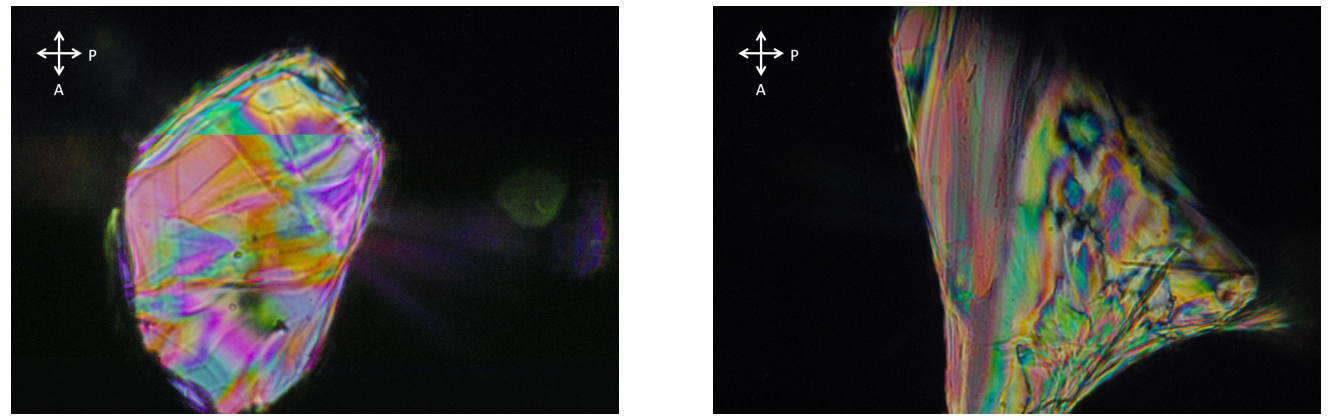

Fig. 4.3 POM images of $(\mathbf{1 0})$ at $140^{\circ} \mathrm{C}$ with $100 \mathrm{x}$ magnification.

case this crystalline solid melted directly into the isotropic liquid and underwent crystallisation directly from this liquid to the gold crystalline solid at similar temperatures to that seen in the DSC trace. Thus no liquid crystalline behaviour was present. Further evidence of this, was that the heats of transition associated with melting and crystallisation in the DSC trace were similar, indicating that it was unlikely there were any other transitions occuring in these derivatives.

It was thought that these tails may not introduce enough entropy into the system to overcome the dominating enthalpy of crystallisation induced by the central rigid core. Another factor to consider was that the phenyl rings present on each side of the anthracene core which sit perpendicular to the plane of the central anthracene core, may disrupt the close range selfassembly required by a mesophase preventing its formation.

If it was a result of the latter, then additional tails introduced in common liquid-crystalline-inducing motifs would provide sufficient entropy and induce liquid crystalline behaviour. ${ }^{51}$ Thus, a new class of hexacatenar 9,10bis(3,4,5-tri(alkyloxy)phenyl)anthracenes were synthesized which to the best of our knowledge have never previously been synthesized. 


\subsection{Synthesis of Hexacatenar DPA Deriva- tives}

In the synthesis of the hexacatenar DPA derivatives, alkyl chains were attached at the 3,4,5 positions of the outer phenyl rings of DPA. It was thought the presence of these alkyl tails could introduce sufficient entropy into the system in order to produce liquid crystalline phases. The 3,4,5-tri(alkyoxy)phenyl group is not uncommon amongst liquid crystalline molecules and is seen as a motif which has been used to induce such behaviour previously. 1915159 It must be acknowledged that there have been few examples of liquid crystalline derivatives based on DPA, thus the synthesis of novel derivatives and the analysis of their phase behaviour provides valuable information as to the requirements of liquid crystallinity of DPA derivatives. $\frac{9144}{9}$

The synthesis of such derivatives has not been previously reported to the best of my knowledge. As a consequence the synthesis had to be optimised, compared to the known synthesis of the previous dicatenar derivatives. The presence of such a large number of alkyl chains made the chemistry and reactivity involved remarkably different. The starting material used, 5-bromo3,4,5-trimethoxybenzene, was commercially available.

A Suzuki coupling was attempted between this starting material and anthracene-9,10-diyldiboronic acid (synthesized not with great yield) directly. $\underline{64}$ However, while anthracene was produced in great quantity, no desired product was produced. From this reaction, it was clear that the phenyl group must be the chemical derivative that contains the boronic acid.

There were two possible routes for the desired synthesis. Either tails were added before the Suzuki reaction or they were added as the final step. The former was chosen (Scheme 4.2) as it offered ease of deprotection, where only 
three such methyl groups must be cleaved from the molecule in contrast to six in the latter. Boron tribromide is the typical reagent of choice when deprotections of three consecutive methoxy groups are involved, however is not easily obtained or synthesized as it is an extremely hazardous reagent.19/51/60 Hydrobromic acid was used as the first choice in this synthesis. However this reagent produced a mixture of cleaved products. Boron tribromide was the only reagent powerful enough to perform this step.

Two different syntheses of this reagent were trialled, the first involving reaction between potassium fluoroborate and aluminium tribromide to yield boron tribromide, the second involved reaction of pelleted boron and bromine at $700{ }^{\circ} \mathrm{C}$. Both routes were extremely low yielding (ca. $\left.1 \%\right)$. The former route produced sufficient material for one such reaction. ${ }^{65166}$ The product of 5-bromo-1,2,3-trihydroxybenzene (11) was obtained with a near $100 \%$ yield. The next step involved the attachment of octyl chains via Williamson ether synthesis to yield 5-bromo-1,2,3-tri(octyloxy)benzene (12).

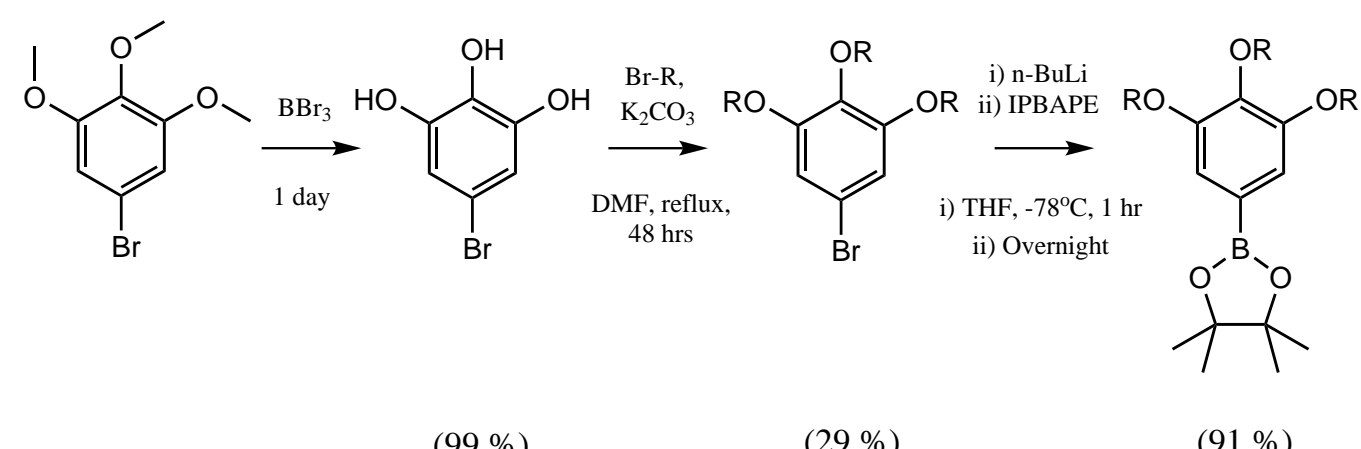

Scheme 4.2 Scheme of the synthesis of 3,4,5-trimethoxyphenyl boronic acid pinacol ester.

This material was then reacted with n-butyllithium at $-70^{\circ} \mathrm{C}$, followed by addition of 2-isopropoxy-4,4,5,5-tetramethyl-1,3,2-dioxaborolane (IPBAPE) to yield the boronic acid pinacol ester (13). This was chosen over the boronic 
acid for the decreased reactivity towards water and increased stability of the molecule over time. The final reaction in this sequence was a Suzuki coupling between (13) and 9,10-dibromoanthracene. However, no desired product was obtained. It was thought that the catalyst was unable to function properly in the presence of additional steric hindrance and extreme solubility induced by the three alkyl tails.

As a result the second synthetic route, in which the tails were added as the final step, provided the most promising option of synthesizing these hexacatenar derivatives (Scheme 4.3). The first step was the synthesis of 3,4,5trimethoxybenzeneboronic acid pinacol ester (14). The reagent 5-bromo1,2,3-trimethoxy benzene was reacted with n-butyllithium at $-70^{\circ} \mathrm{C}$, followed by addition of 2-isopropoxy-4,4,5,5-tetramethyl-1,3,2-dioxaborolane (IPBAPE). $\underline{51}$

This time the Suzuki reaction between the pinacol ester and 9,10-dibromo anthracene proceeded to yield 9,10-bis(3,4,5-trimethoxyphenyl)anthracene (15) with a reasonable yield (ca. $50 \%$ ). This yield was much lower than those obtained with the Suzuki reactions involved with the synthesis of dicatenar derivatives. This was due to the presence of two additional meta methoxy groups, which are known to be deactivating, thus increased reaction times were required.

The next step involved cleavage of the six methoxy groups present on the central DPA core. As mentioned previously boron tribromide is the most common reagent used, but could not be synthesized in the quantities required for this reaction. The compound (15) was sent to the University of York, where my third supervisor, Prof. John Spencer was on research and study leave working in a research group that focusses on the synthesis of liquid crystals. Boron tribromide is used frequently there, so the deprotection was completed where 9,10-bis(3,4,5-trihydroxyphenyl)anthracene (16) was 
obtained in almost $100 \%$ yield.<smiles>COc1cc(Br)cc(OC[C@@H](C)C(F)F)c1OC</smiles><smiles>COc1cc(B2OC(C)(C)C(C)(C)O2)cc(OC)c1OC</smiles>

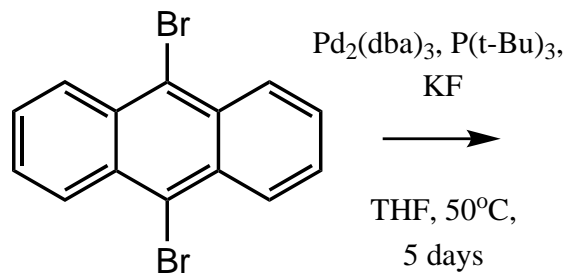

ii) Overnight

$(99 \%)$

14<smiles>COc1cc(-c2c3ccccc3c(-c3cc(OC)c(OC)c(OC)c3)c3ccccc23)cc(OC)c1OC</smiles>

$(51 \%)$

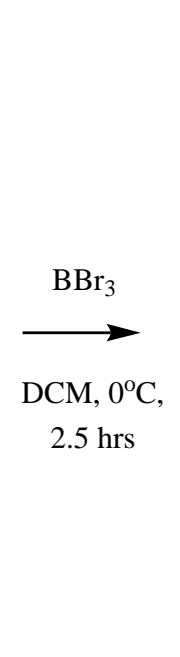<smiles>Oc1cc(-c2c3ccccc3c(-c3cc(O)c(O)c(O)c3)c3ccccc23)cc(O)c1O</smiles><smiles>[R]c1cc(-c2c3ccccc3c(-c3cc([R7])c(O)c([R])c3)c3ccccc23)cc([R])c1O</smiles>

15

$$
\mathrm{R}=\mathrm{C}_{10} \mathrm{H}_{21}(11 \%) \quad \mathbf{1 7}
$$

$$
\mathrm{R}=\mathrm{C}_{16} \mathrm{H}_{33}(5 \%)
$$

Scheme 4.3 Scheme of the synthesis of hexacatenar derivatives of DPA

Both bromodecyl and bromohexadecyl chains were attached via Williamson 
ether syntheses to produce the 9,10-bis(3,4,5-tri(decyloxy)phenyl)anthracene (17) and 9,10-bis(3,4,5-tri(hexadecyloxy)phenyl)anthracene (18) in good yield.

\subsection{Phase Behaviour of Hexacatenar DPA Deriva- tives}

The synthesized derivatives, (17) and (18) were investigated for mesomorphic behaviour. The crystalline solids of both compounds were made up of microscopic needles at room temperature and remained so up until the melting transitions of these compounds.

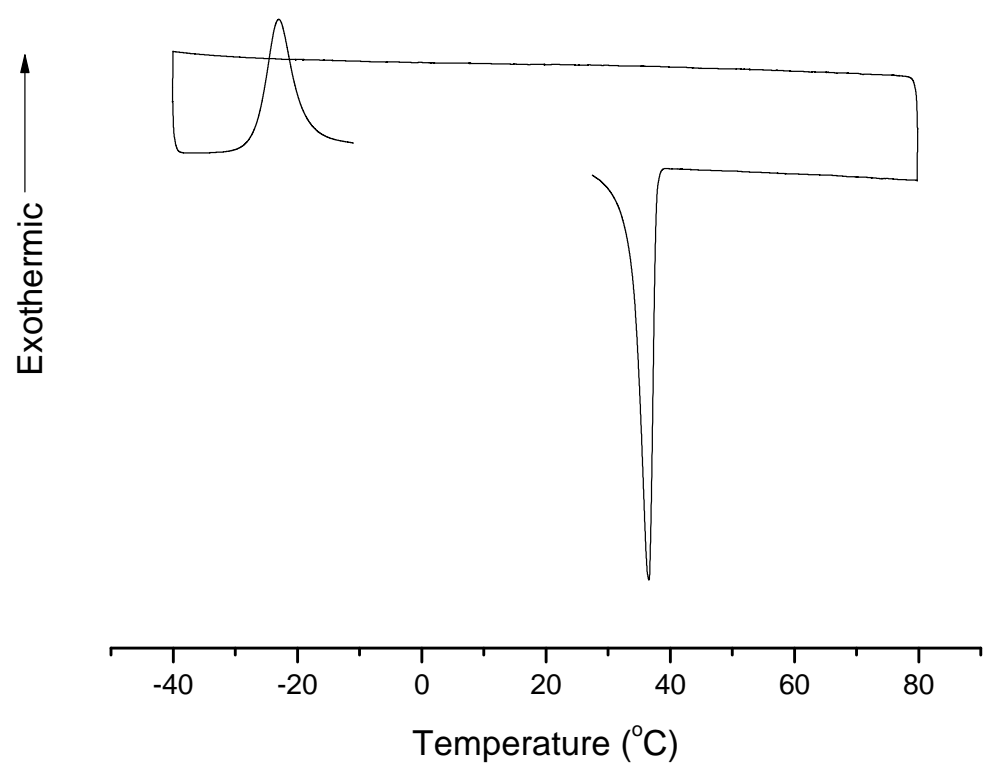

Fig. 4.4 DSC plot for (17) at heating and cooling rates of $1^{\circ} \mathrm{C} \mathrm{min}^{-1}$. 
The product (17) was the first to be tested, which showed some interesting and unique behaviour. On the preliminary testing of this compound, it was found that this compound did not crystallise out at temperatures above $0^{\circ} \mathrm{C}$ on cooling. Only one peak corresponding to melting was observed in the original DSC trace at $37^{\circ} \mathrm{C}$.

On further investigation, the temperature programme was adjusted so that after heating to $70^{\circ} \mathrm{C}$, the compound was cooled to $-40^{\circ} \mathrm{C}$ and heated back to room temperature at a rate of $1^{\circ} \mathrm{C} \mathrm{min}^{-1}$ (Fig. 4.4). Though, it was only on reheating from $-40{ }^{\circ} \mathrm{C}$ that a peak was observed $-23^{\circ} \mathrm{C}$.

On direct observation of the sample under the POM microscope, the sample is seen to melt directly into the isotropic liquid, close to that observed in the DSC trace at $37.1^{\circ} \mathrm{C}$, and crystallises back into a gold crystalline solid, but forms no liquid crystal phases.

This compound exhibits extreme supercooling and will remain a liquid for several days before crystallisation will eventually occur. This is a unique and new property as it the only derivative to undergo such supercooling and there have only been a few derivatives previously reported of DPA that are liquids at room temperature. $67 / 68$

One such neat liquid DPA derivative previously reported has been used in conjugation with a liquid PdOEP sensitizer and has successfully resulted in upconversion in this neat liquid state. ${ }^{67}$ It then follows that the neat liquid of (17) could also facilitate this process given inclusion of a sensitizer. There have been studies on the distance required for electron transfer in upconversion using the Perrin approximation for sensitizer and emitter pairs such as PtOEP and DPA. ${ }^{622}$ The Perrin approximation is used to overcome difficulties in obtaining spectral overlap integrals for forbidden transitions and proper wavefunctions for such molecules. ${ }^{6 / 22}$ The Dexter radii $\left(R_{0}\right)$ is 
the distance on which efficient electron transfer can take place, provided the distance between the molecules is smaller than or equal to such distance.22 This distance for PtOEP and DPA was determined by measuring the PL of PtOEP as a function of DPA concentration in a frozen solution. This was fitted using an equation to yield a $26.5 \AA$ Dexter radius. ${ }^{22}$

The distances between the DPA cores in the previously reported neat liquid DPA derivative were examined by XRD. ${ }^{67}$ These cores exhibited some sort of long range order and were found to be on average $21 \AA$ apart without the inclusion of the sensitizer. $\frac{67}{}$ It can be assumed that $(\mathbf{1 7})$, given its similarity in structure may have a similar distance between molecules. This is smaller than the Dexter radius and given the small amount of sensitizer needed (2 wt \%) this distance would not be significantly altered. It then follows that upconversion is possible given the success with other similar derivatives.

The product (18) also shows interesting behaviour; however it was difficult to remove the residual acetone and water from the sample due to the large molecular weight and size of this molecule. As a result multiple peaks were observed in the DSC plot. On the first measurement of a new sample, only one peak is seen, but when a second cycle of the same temperature regime is performed, three peaks are observed (Fig. 4.5). This behaviour was consistent and was seen again as the measurements were cycled. The transition temperatures (Table 4.2) obtained correspond to melting of the crystalline solid but also to residual solvent leaving the material.

Of the three peaks observed, it is likely that the first at $47.59{ }^{\circ} \mathrm{C}$ corresponds to a real transition of the material, this is because it has the same enthalpy as the large peak seen on cooling. The second two peaks correspond to the boiling points of free and bound residual acetone in the system at $47.59{ }^{\circ} \mathrm{C}$ and $56.63^{\circ} \mathrm{C}$. Due to this presence of acetone, melting of the 


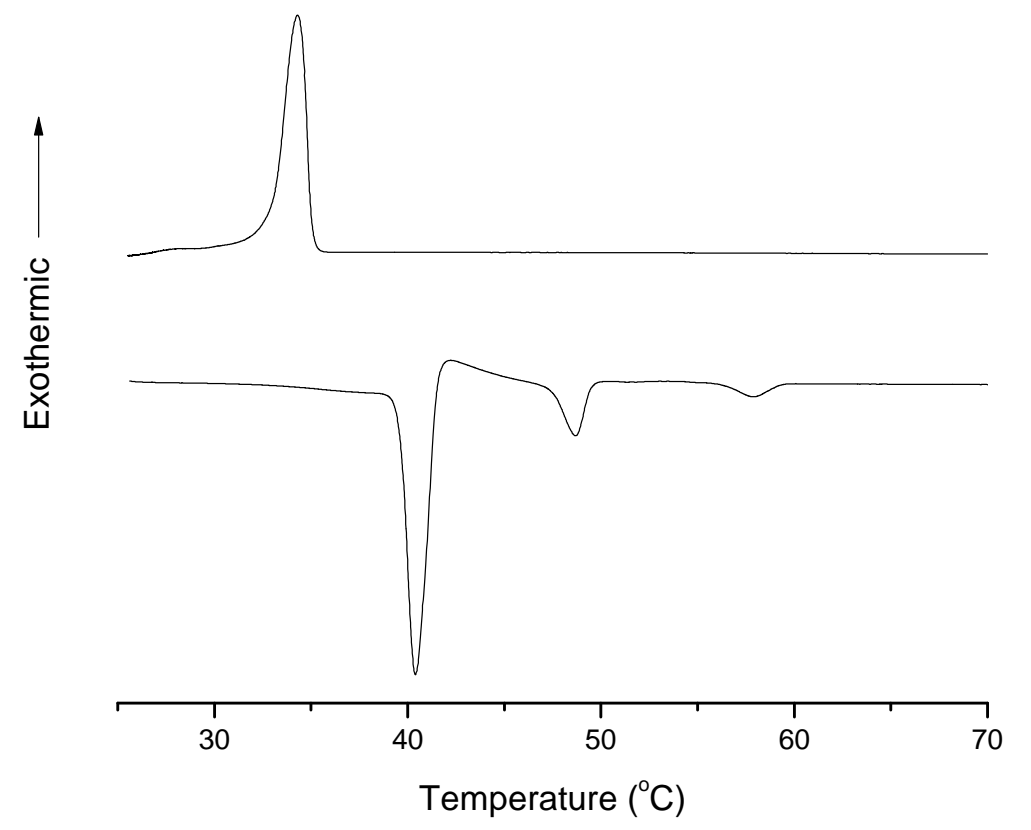

Fig. 4.5 DSC plot of $(\mathbf{1 8})$ at a rate of $1^{\circ} \mathrm{C} \mathrm{min}-1$. 
solid is not seen directly under the microscope. The presence of acetone prolongs the melting of the crystal structure which results in defects in the system (Fig. 4.6). The product (18) shows no evidence of liquid crystalline behaviour.

Table 4.2 DSC Peaks for (18).

\begin{tabular}{cccc}
\hline \hline Round & Transition & Temperature ${ }^{\circ} \mathrm{C}$ & Heat $/ \mathrm{Jg}^{-1}$ \\
\hline 1 & Melting & 54.54 & -93.72 \\
- & Crystallisation & 35 & 64.80 \\
2 & Melting & 39.46 & -64.99 \\
- & Acetone & 47.59 & -21.53 \\
- & Acetone & 56.63 & -4.65 \\
- & Crystallisation & 35.29 & 62.70 \\
\hline
\end{tabular}
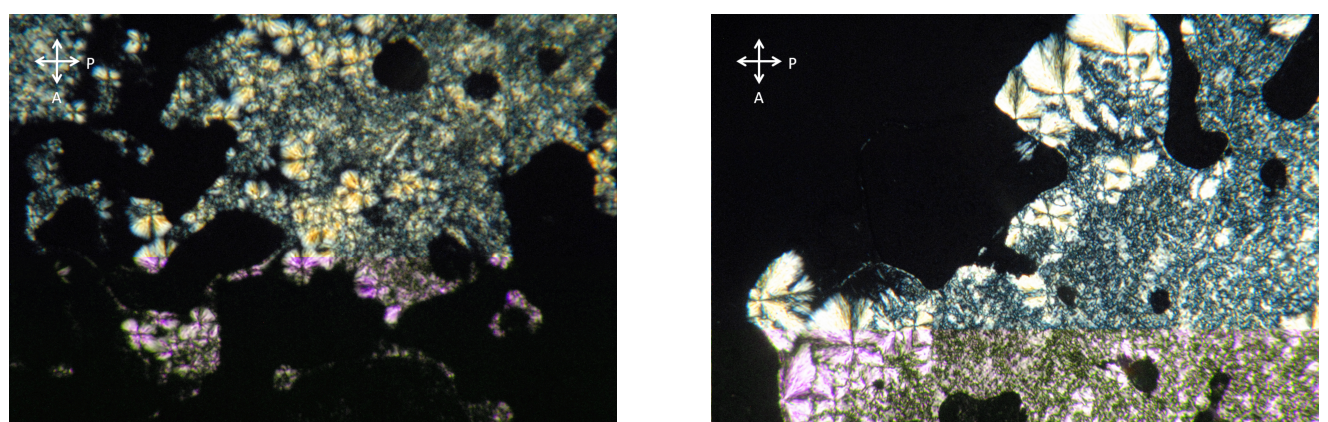

Fig. 4.6 POM images of the crystalline solid of (18) at $44.1^{\circ} \mathrm{C}$ at $40 \mathrm{x}$ magnification.

\subsection{Conclusions}

The resulting phase behaviour has given new insight into the requirements for liquid crystallinity in compounds described in this chapter. The central DPA core was extended with addition of alkyl chains in order to elongate 
the core and give it a lath-like shape. These chains were varied by number and in length however this did not result in the formation of a mesophase. The phenyl rings sit perpendicular to the central anthracene ring due to repulsion of hydrogen atoms at positions 2- and 6- on the phenyl rings and the 1-, 4-, 5- and 8- positions on the anthracene core. This could disrupt the ease of close packing needed for a mesophase. It is also possible that the molecule was not elongated sufficiently to result in the formation of a liquid crystal phase. Future work could focus on elongation of the central core with a phenyl ring which has previously resulted in the formation of only one liquid crystal. ${ }^{9}$ Despite this, a novel material which undergoes extreme supercooling was synthesized; 9,10-bis(3,4,5-tri(decyloxy)phenyl)anthracene. This material remains a liquid for several days before crystallisation occurs. There have been a few examples of liquid 9,10-diphenylanthracene's reported, but this is the first instance of any such supercooling behaviour. ${ }^{6768}$ It may be an interesting emitter material for upconversion, as the molecules in a neat liquid are not ordered, but are extremely close to each other and able to diffuse through solution. These properties may be able to enhance the process of upconversion when blended with an appropriate sensitizer such as palladium octaethylporphyrin. 


\section{Chapter 5}

\section{9,10- \\ Bis(phenylethynyl)anthracene (BPEA) Derivatives}

Three derivatives of 9,10-bis(phenylethynyl)anthracene (BPEA) were synthesized, alkyl tails were attached with or without a phenyl linker. BPEA does not exhibit mesomorphic behaviour itself, thus, in order to overcome the enthalpy of crystallisation of the rigid central core long flexible alkyl chains were attached which provide entropy to the system. However, this molecule has one important advantage over DPA in that the triple bonds present allow the attached phenyl rings to sit planar with the central anthracene unit, which may allow closer ease of self assembly and may result in the formation of a liquid crystalline phase.

Two dicatenar BPEA derivatives were synthesized where the attached alkyl chain was varied in length; tails of 8 and 16 carbons were used. The attachment of alkyl chains directly to the central core in contrast to exten- 
sion of the central core by further aromatic rings provided the most direct approach to induce liquid crystalline behaviour without significantly altering the photophysical properties of the DPA dye.

9,10-bis(biphenyl-4-ylethynyl)anthracene (BBPEA) was synthesized, in the extension of the BPEA core by two phenyl rings and substituted octyl chains. There have been several examples previously where the BBPEA core with attached pentyl chains has yielded a liquid crystalline derivative. ${ }^{8}$ It was expected that this derivative would almost certainly give rise to the formation of a liquid crystalline phase.

In this chapter synthetic routes for each derivative will be detailed, as well as the phase behaviour for each derivative, though the full experimental and characterisation information will not be discussed. The full synthetic and experimental procedures and compound characterisation can be found in the experimental chapter.

\subsection{Synthesis of Dicatenar BPEA Derivatives}

The synthesis of these derivatives involved four main steps (Scheme 5.1. In contrast to the syntheses of DPA derivatives, tails were attached as the first step, due to the more accomodating nature of the Sonogashira reaction. The molecule 4-hydroxyphenylbromide was reacted with bromooctane at elevated temperatures to produce 4-(octyloxy)phenylbromide (19) as a colourless oil. All intermediates synthesized with this length chain were oils, as a consequence all products were purified using column chromatography on silica. Compound (19) was reacted with trimethylsilylacetylene (TMSA) in the presence of tetrakis(triphenyl phosphine)palladium(0) and copper iodide, which yielded 4-(octyloxy)phenyltri methylsilylacetylene (20) in reasonable 
yield (ca. $60 \%)$.

It is essential that this material is purified to remove traces of catalyst before reaction with potassium hydroxide in the deprotection of the trimethylsilyl group. Otherwise, homo-coupling will occur between triple bonds and the desired product will not be produced. Thus, (20) was purified through column chromatography on silica before reaction with potassium hydroxide, which yielded 4-(octyloxy)phenylacetylene (21).

The compound (21) was reacted with 9,10-dibromoanthracene in the presence of tetrakis (triphenylphosphine)palladium(0) and copper iodide to yield 9,10-bis(4-(octyloxy)phenylethynyl)anthracene (22), which was then purified by column chromatography on silica and recrystallised multiple times. Unfortunately, this was not completely effective and there was still grease left in the material that was not able to be removed. This occurred in the proton NMR at 1.2-1.5 and 0.89 ppm. However, measurements of this impure compound were still performed to obtain information of the phase behaviour.

The synthesis in the same manner, was trialled for dodecyl and hexadecyl derivatives. Due to time constraints, 9,10-bis(4-(hexadecyloxy)phenylethynyl) anthracene (23), was synthesized by Dr. K. Allan, using similar methods to the octyl derivative summarised above. $\frac{69}{6}$ In contrast to compound $(\mathbf{2 2})$, compound $(\mathbf{2 3})$ was able to be obtained as a pure crystalline powder. 


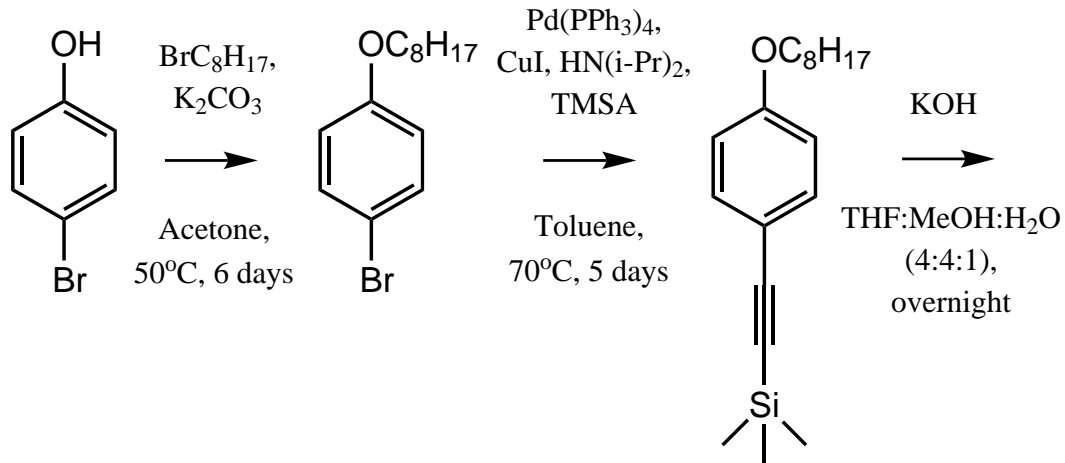

$(58 \%)$

$(61 \%)$
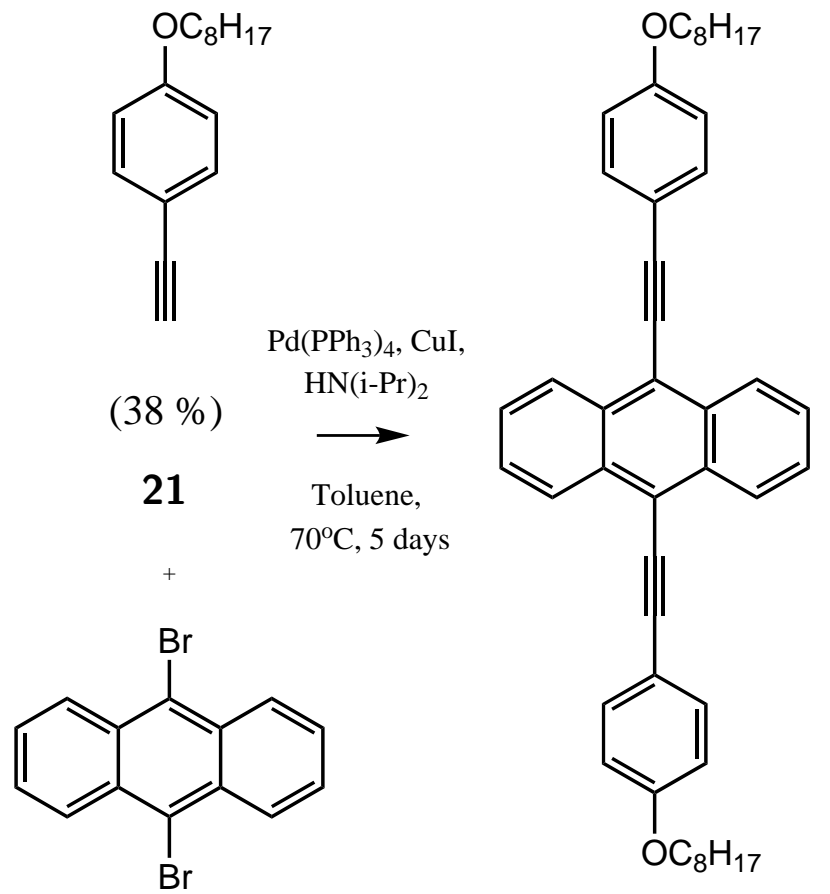

$(20 \%)$

Scheme 5.1 Scheme of the synthesis of (22). 


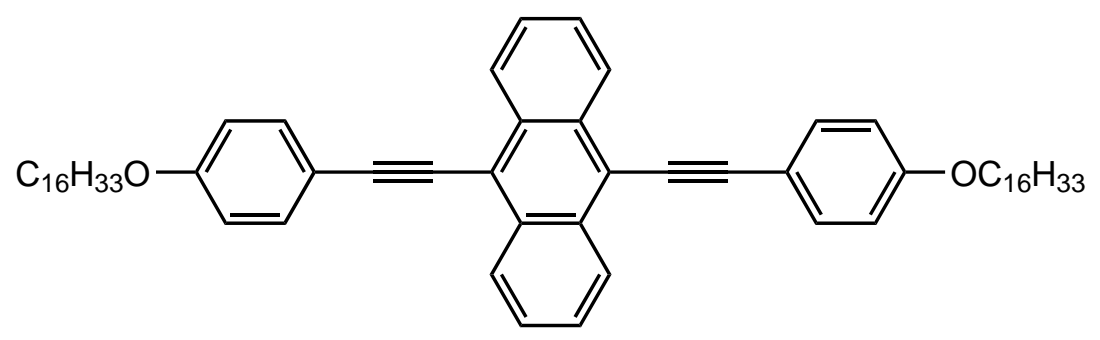

23

Scheme 5.2 Structure of (23).

\subsection{Phase Behaviour of Dicatenar BPEA Deriva- tives}

Compounds (22) and (23) were investigated for mesomorphic behaviour. The DSC trace of $(\mathbf{2 2})$ revealed a transition occurring at $135^{\circ} \mathrm{C}$, followed by decomposition around $150^{\circ} \mathrm{C}($ Fig. 5.1). Under observation of this compound under the microscope, a yellow crystalline solid was seen melting at $130^{\circ} \mathrm{C}$, which was followed by the melting of a red solid and direct decomposition of the material at $157.6^{\circ} \mathrm{C}$ in which the solid went irreversibly black.

This material may be exhibiting degrees of polymorphism or it may just be a result of the impurities contained within the sample. However, there were no liquid crystalline phases formed.

The DSC trace of (23) showed one peak on heating at $136.76{ }^{\circ} \mathrm{C}$ with an enthalpy of $-135.02 \mathrm{Jg}^{-1}$. One peak was observed on cooling at $132.68^{\circ} \mathrm{C}$ with an enthalpy of $138.75 \mathrm{Jg}^{-1}($ Fig. 5.2). Upon cycling, these measurements were reproducible. The melting and crystallisation transitions also occurred around the same temperatures on observation under the polarised optical microscope. The solid melted directly into the isotropic phase, and 


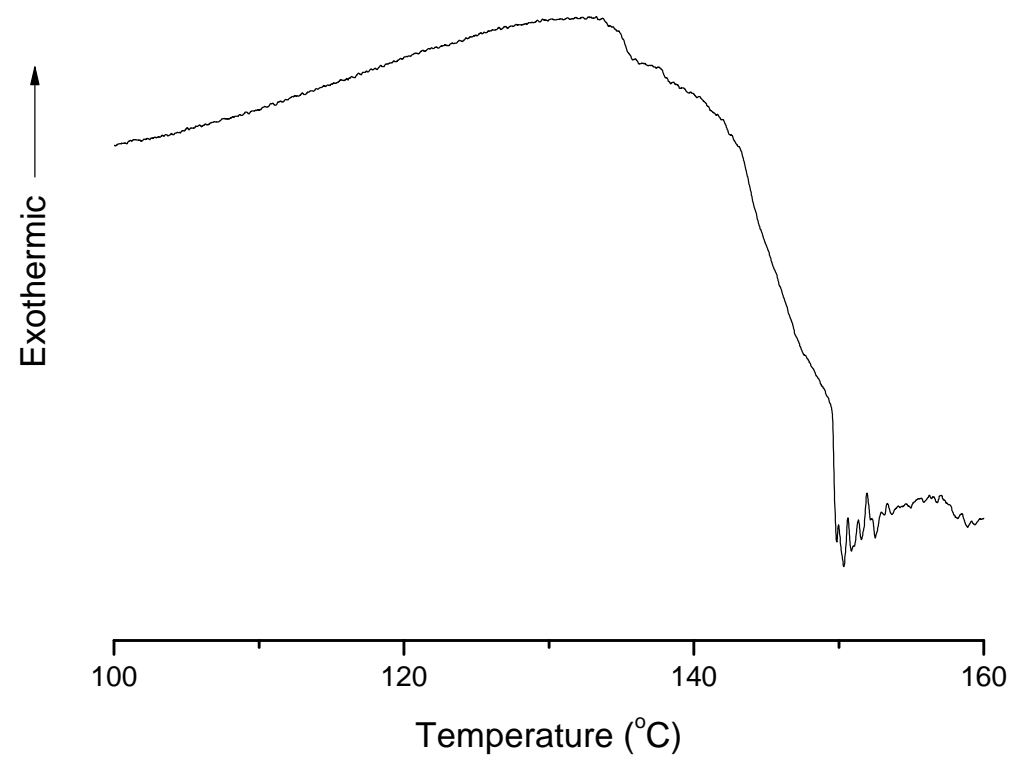

Fig. 5.1 DSC plot for $(22)$ at a rate of $1^{\circ} \mathrm{C} \mathrm{min}^{-1}$. 
crystallisation was observed directly, without the formation of a mesophase.

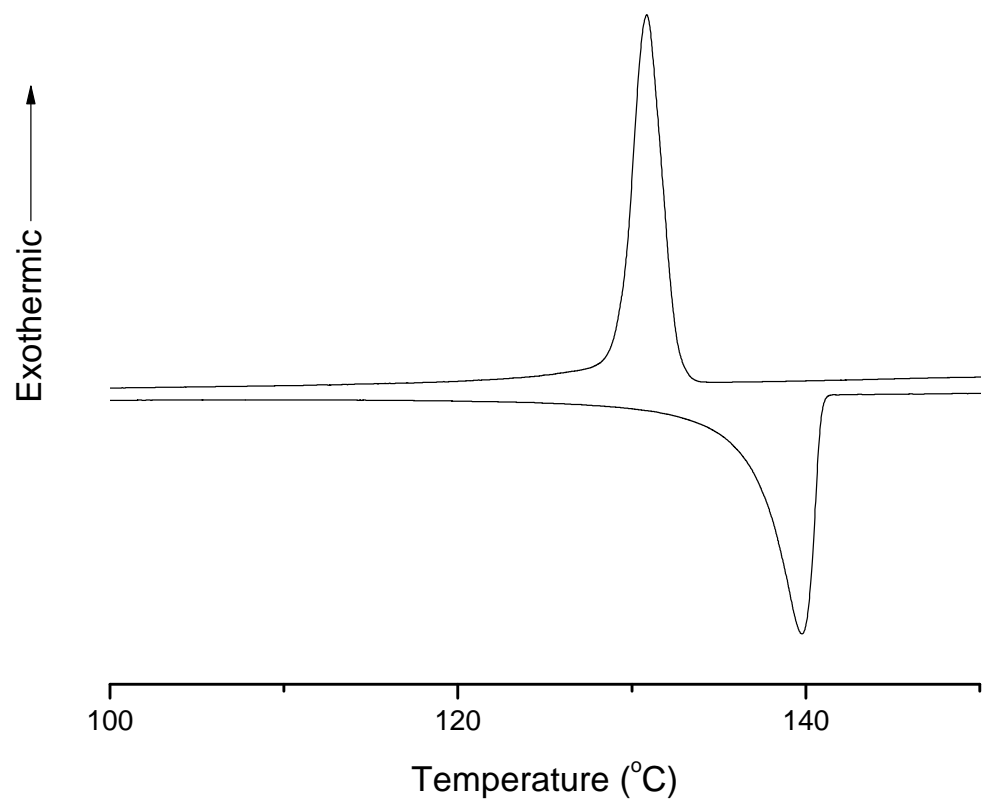

Fig. 5.2 DSC plot for $(\mathbf{2 3})$ at a rate of $1^{\circ} \mathrm{C} \mathrm{min}^{-1}$.

It was expected that through attachment of alkyl chains to the central BPEA core, liquid crystalline behaviour of the resulting compounds would be observed. Three novel compounds have been synthesized, but none have exhibited liquid crystalline behaviour. It has been found in the literature that similar compounds synthesized, such as in which the symmetric nature of the compound favoured the crystalline state. ${ }^{8}$ However, asymmetric derivatives with attached alkyl chains of different length on either side do result in liquid crystalline behaviour which, if direct substitution is desired may be an area which could be explored further. 


\subsubsection{Phase Behaviour of the Dicatenar BBPEA Deriva- tive}

The compound 9,10-bis(4'-(octyloxy)biphenyl-4-ylethynyl)anthracene (24) (Scheme 5.3) was supplied by Dr. K. Allan and its synthesis was based on methods found in the literature. $\underline{69} 71$

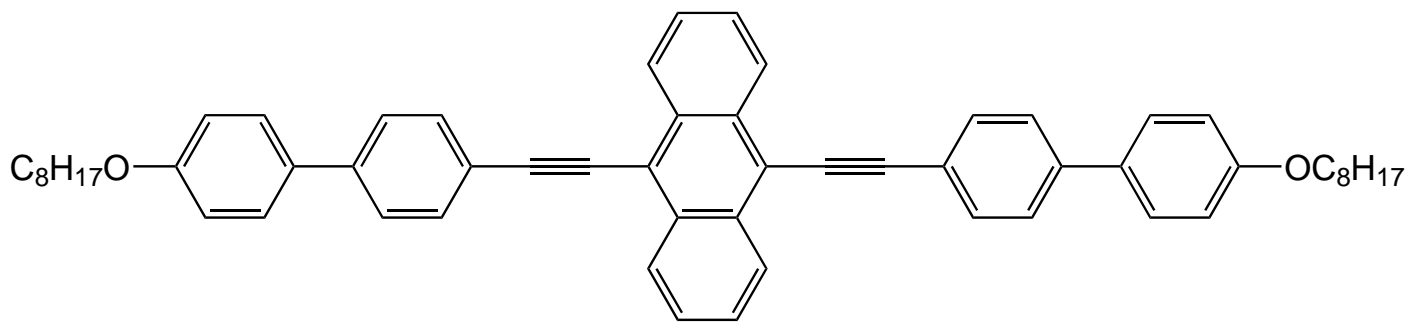

24

Scheme 5.3 Structure of (24).

The DSC trace shows one main transition on heating at $209.54^{\circ} \mathrm{C}$ with an enthalpy of $-43.04 \mathrm{Jg}^{-1}$ and on cooling shows another transition at $198.72^{\circ} \mathrm{C}$ with an enthalpy of $34.88 \mathrm{Jg}^{-1}$ (Fig. 5.3). On cooling there is a smaller peak observed around $180^{\circ} \mathrm{C}$. With observation under the microscope, this smaller peak could not be identified with a transition occurring in the material. It may correspond to residual solvent in the material.

After observation under the microscope it is seen that the first transition corresponds to the formation of a liquid crystalline nematic phase at $209.55^{\circ} \mathrm{C}$. When heated further the compound does not form an isotropic phase but undergoes direct decomposition at $270{ }^{\circ} \mathrm{C}$ (Fig. 5.4). This has also been seen in a compound reported in the literature, where pentyl chains were directly attached to the BBPEA core. Decomposition was also found to occur at $270^{\circ} \mathrm{C} . \underline{8}$ 


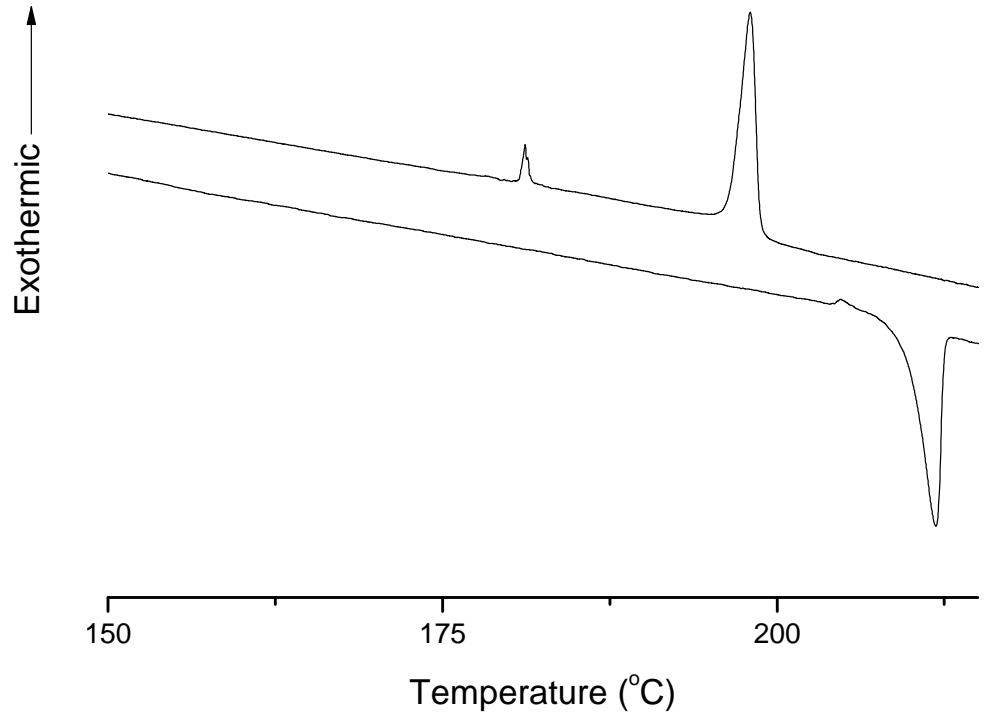

Fig. 5.3 DSC plot for $(\mathbf{2 4})$ at a rate of $1^{\circ} \mathrm{C} \mathrm{min}^{-1}$. 
If not heated to decomposition, on cooling the material undergoes crystallisation into a mosaic-like crystalline solid (Fig. 5.5). Thus a liquid crystal based on BBPEA has been obtained, which may prove an interesting material for facilitation of upconversion.
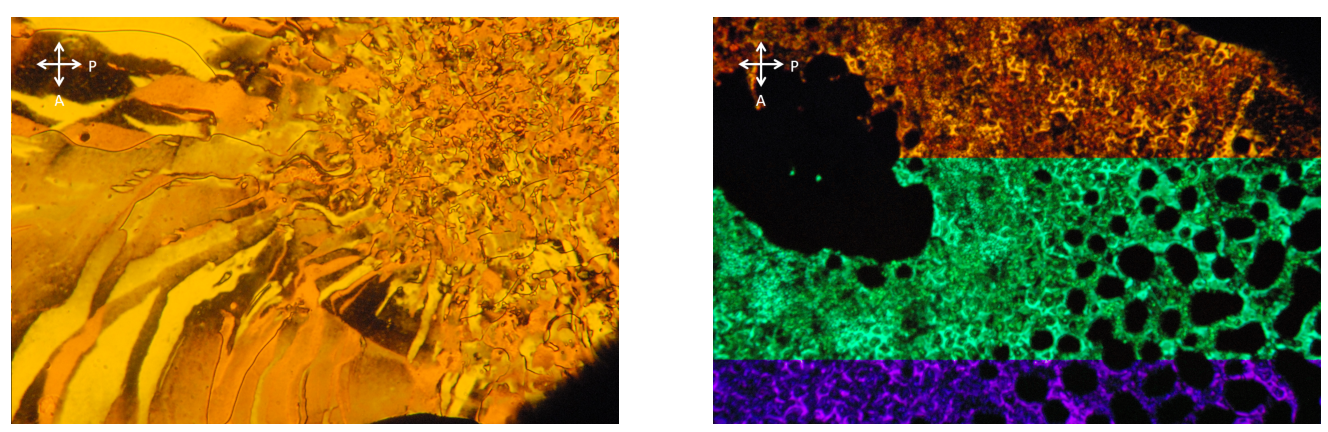

Fig. 5.4 POM Images of the nematic phase of $(\mathbf{2 4})$ at $240{ }^{\circ} \mathrm{C}$ (left) and decomposition of this material at $270{ }^{\circ} \mathrm{C}$ (right) at $40 \mathrm{x}$ magnification.

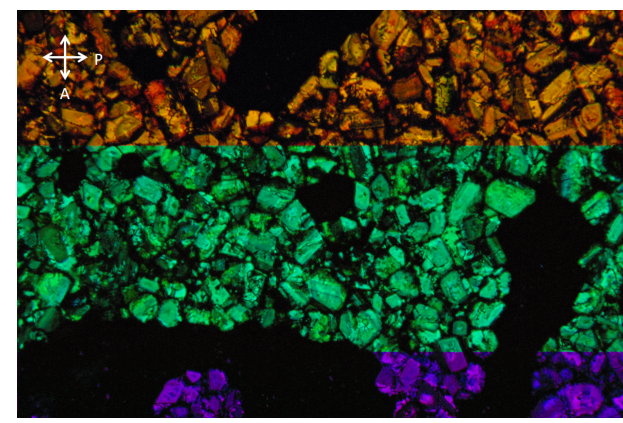

Fig. 5.5 Mosaic Crystalline Solid of $(\mathbf{2 4})$ at $198^{\circ} \mathrm{C}$ at $40 \mathrm{x}$ magnification.

The temperature at which the liquid crystalline phase forms is very high, which may make measurement of the photophysical properties of this material difficult. 


\subsection{Conclusions}

Three new derivatives of BPEA have been synthesized. One such compound, 9,10-Bis(4'-(octyloxy)biphenyl-4-ylethynyl)anthracene exhibits the formation of a liquid crystalline phase at elevated temperatures. There were previous BPEA derivatives (outlined in chapter 3) which exhibited liquid crystalline behaviour which gave insight as to which structural motifs for this core resulted in liquid crystallinity. ${ }^{8 / 50}$ In this chapter, the central BPEA core was also extended accordingly with alkyl chains in order to elongate the core and give it a lath-like shape. The BPEA core is physically longer than that of DPA due to the presence of ethynyl linkage groups to the central core, which create a more planar, more lath-like molecule. However, this direct symmetric substitution of alkyl chains did not result in the formation of a mesophase. A reason for this may be the molecule needs to be elongated further. Extension of the central core by symmetric addition of aromatic rings was required for the formation of mesophases in accordance with previous literature. .850 


\title{
Chapter 6
}

\section{Spectroscopy of}

\section{9,10-Diphenylanthracene}

\author{
(DPA) and
}

\section{9,10-Bis(phenylethynyl)}

\section{anthracene (BPEA) Derivatives}

The photophysical properties were investigated for all the dye derivatives synthesized in previous chapters. Absorption and emission spectra for each derivative were obtained. In addition, compounds (17) and (24) were studied in thin films. It was important to determine whether these liquid phase derivatives had the appropriate properties for use a materials to facilitate upconversion. Ideally, the photophysical properties, such as absorption and emission spectra will not be altered from the original dye molecule. Also, the liquids would retain the fluidity of the liquid or liquid crystal phase on inclusion of a sensitizer. 
The films were blended with 2 wt\% sensitizer, which in this case was PdOEP. This sensitizing agent was used with both DPA and BPEA dyes. DPA as discussed previously is the most common emitter used in conjunction with PdOEP. The emitter commonly used with BPEA, $\mathrm{PdPh}_{4} \mathrm{TBP}$, is not easily synthesized or sourced due to high cost. Although the use of PdOEP with BPEA does not provide the largest energy gain, it can be satisfactorily used as a replacement for proof of concept.

\subsection{Upconversion in Solution}

Upconversion was replicated with each sensitizer and emitter couple, with PdOEP and the original emitter molecule before investigating new materials for upconversion. It was important to ensure that upconversion in solution could be reproduced, and the experimental details ascertained.

Sample preparation for solution upconversion is extremely important. The first thing that was considered was the way in which oxygen was removed. Triplet states are extremely sensitive to the presence of oxygen in ppm amounts. Oxygen interacts with the triplet state of the metallated donor, quenching the phosphorescence by producing the donor ground state and singlet oxygen. $\frac{16 / 17 / 72}{1}$

A number of methods have been utilised by others previously; bubbling inert gasses like nitrogen or argon through the sample, freeze-pump- thaw of the solvent, or preparing samples in a glove box. ${ }^{16}$ Firstly, freeze-pumpthaw was used to degass the upconversion solution of sensitizer and emitter. The method was first tested on a solution of PdOEP. The phosphorescence of the triplet state was not present in the emission spectrum. It was found the apparatus did not fit properly with the cuvette which was directly at- 
tached by a ground glass joint and oxygen was not sufficiently removed from the sample. Thus, the cuvette joint was allowing air to enter the apparatus. Subsequently, upconversion solutions were prepared in the glove box. A solution of sensitizer and emitter in toluene was diluted to the required concentration in a fluorescence cuvette with attached tap. Solvent was removed in vacuo leaving a powder. This was redissolved in toluene in the glove box and the cuvette closed. The cuvette was taken out of the glove box and any required measurements performed.

The absorption and emission spectra for DPA and PdOEP are featured below (Fig. 6.1). The emission of the emitter is of higher energy, shorter wavelengths than the absorption spectra of the sensitizer at longer wavelengths. The excitation wavelength that will excite the sensitizer does not directly excite the emitter DPA, thus any fluorescence observed from DPA will be upconverted fluorescence.

Upconversion could be successfully visually observed in solution for PdOEP and DPA (Fig. 6.2). The laser source, which is green is transformed into a blue line of higher energy as it passes through the sample. This requires high concentrations in solution compared to those required for absorption and emission so that it can be observed visually. The emitter must have a higher concentration than the sensitizer as the process requires efficient quenching of the porphyrin sensitizer by the emitter. Metallated porphyrins such as PdOEP have lifetimes on the order of microseconds. PdOEP has a lifetime of $c a .400 \mu \mathrm{s}$. It is long enough that it serves as a reservoir to the sensitization of emitter molecules and therefore can be present in lower concentrations. In this case it is $10^{-4} \mathrm{M}$ compared with $10^{-3} \mathrm{M}$ of the emitter.

Power dependence is measuring the intensity of the upconverted fluores- 


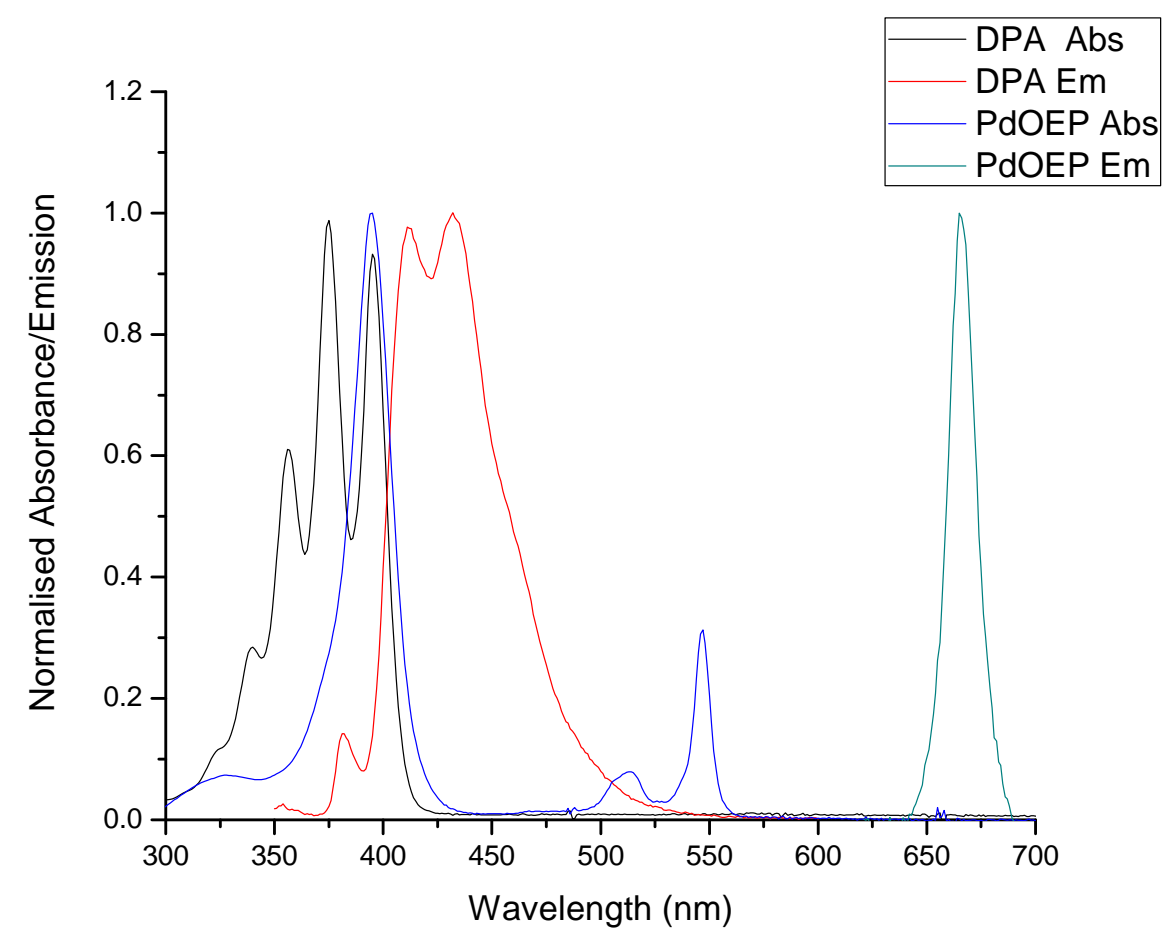

Fig. 6.1 Absorption and emission spectra of DPA and PdOEP in degassed toluene with the following concentrations: DPA (Absorbance:7.5 $\times 10^{-5} \mathrm{M}$,

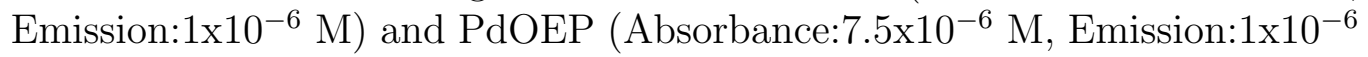
$\mathrm{M})$. 


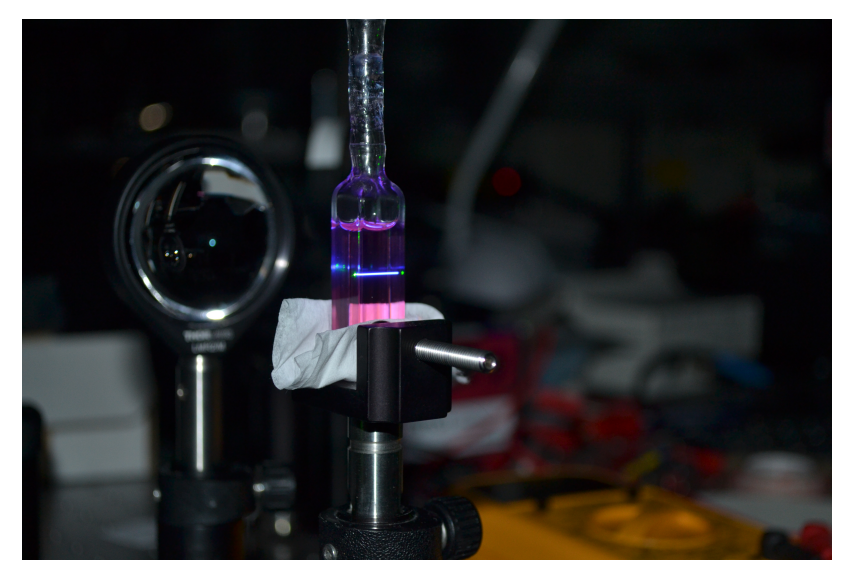

Fig. 6.2 Upconversion of DPA $\left(10^{-3} \mathrm{M}\right)$ and $\operatorname{PdOEP}\left(10^{-4} \mathrm{M}\right)$ in a solution of degassed toluene.

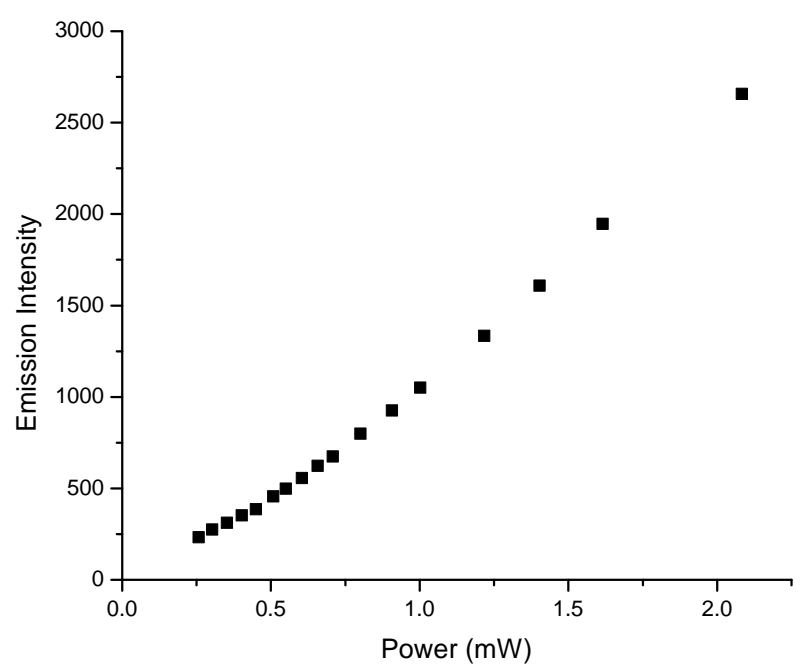

Fig. 6.3 Power dependence of DPA $\left(10^{-3} \mathrm{M}\right)$ and $\operatorname{PdOEP}\left(10^{-4} \mathrm{M}\right)$ in degassed toluene. 
74CHAPTER 6. SPECTROSCOPY OF DPA AND BPEA DERIVATIVES

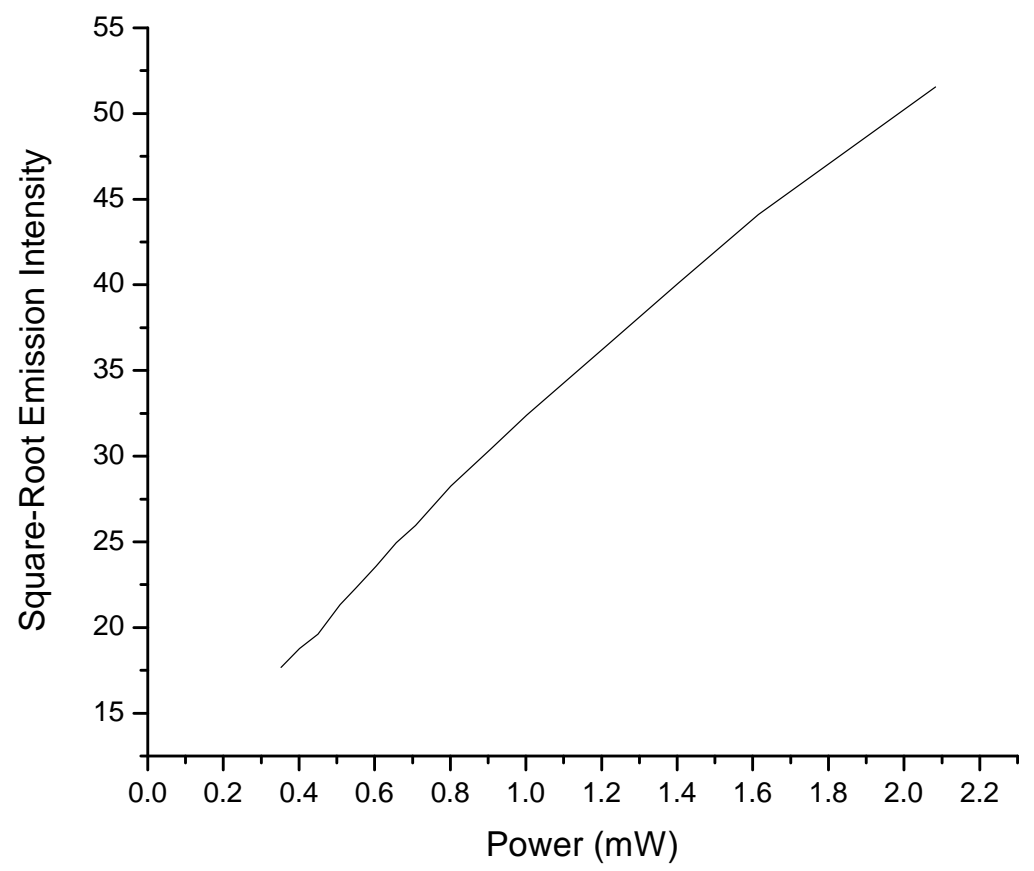

Fig. 6.4 Square-root intensity against incident power for a solution of DPA $\left(10^{-3} \mathrm{M}\right)$ and PdOEP $\left(10^{-4} \mathrm{M}\right)$ in degassed toluene. 
cence as a function of the laser power or power density (excitations per unit volume). This measurement can be used to indicate whether upconversion is occuring. Two photons are used to sensitize to emitter molecules which then undergo TTA to produce a single photon of higher energy. If incident laser power is plotted versus upconverted fluorescence emission intensity, the resulting curve should display a quadratic relationship as in the equation below.

$$
I=P_{i n}^{2}
$$

This measurement was completed for a solution of DPA $\left(10^{-3} \mathrm{M}\right)$ and PdOEP $\left(10^{-4} \mathrm{M}\right)$ in a $1 \mathrm{~cm}$ sealed quartz cuvette. The resulting curve does not appear clearly quadratic $(\overline{\text { Fig. 6.3) }})$. It is suspected that the long path length $(1 \mathrm{~cm})$ resulted in a concentration gradient of excited molecules which when collected obscured the true quadratic curve. It then follows that the square-root of the emission intensity can be plotted against the incident power, which would yield a linear line (Fig. 6.4).

The absorption and emission spectra for BPEA and PdOEP were obtained (Fig. 6.5). PdOEP is not the ideal sensitizer to be used with BPEA as it does not yield the largest energy gain but it is satisfactory to provide proof of concept. The excitation wavelength for the sensitizer does not directly excite the emitter, thus any upconverted fluorescence observed is a direct result of upconversion. It should be noted that this is the first time upconversion has been attempted with BPEA in conjunction with PdOEP as a sensitizer. Upconversion was visualised in solution with concentrations of BPEA $\left(10^{-3} \mathrm{M}\right)$ and PdOEP $\left(10^{-4} \mathrm{M}\right)$ Fig. 6.6). The excitation source was a green laser and a green strip can be visually observed passing through the orange solution. 


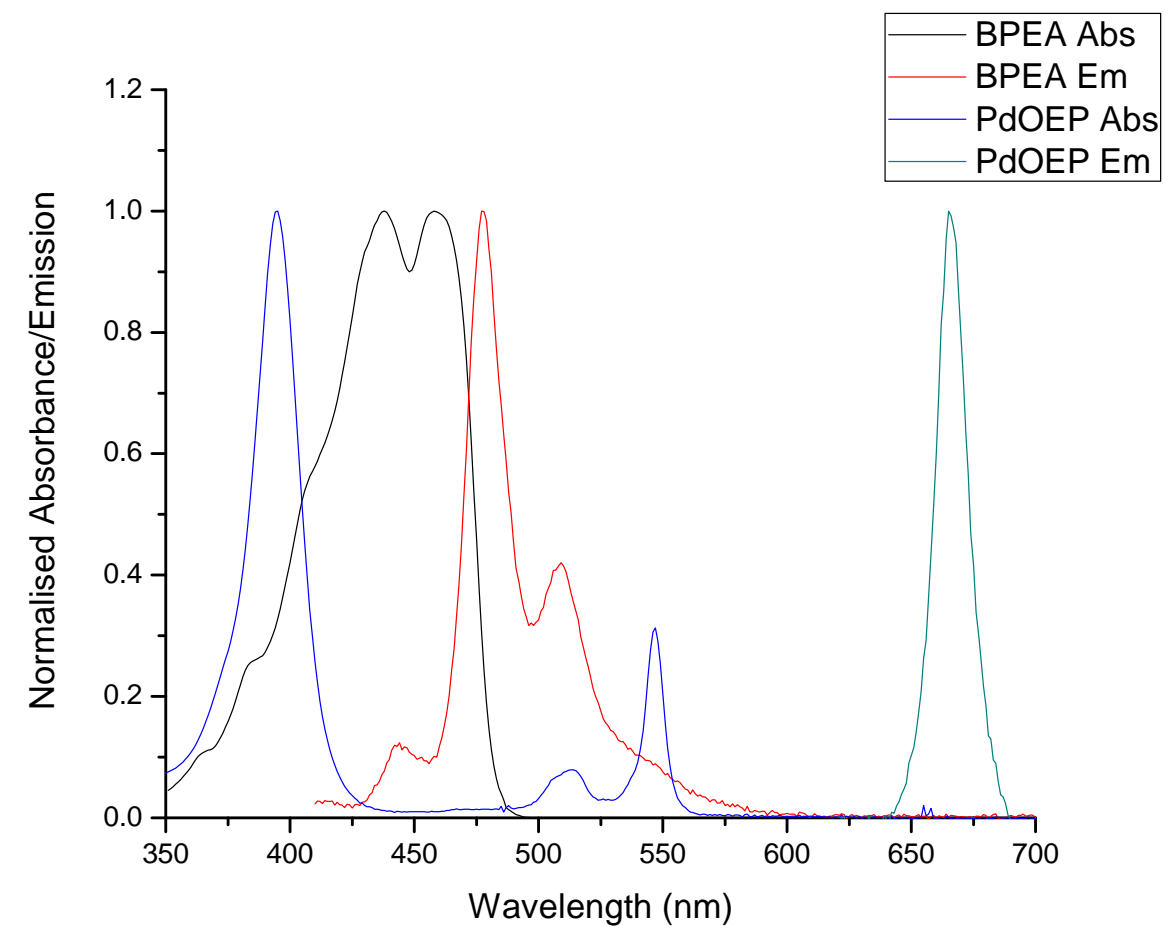

Fig. 6.5 Absorption and emission spectra of BPEA and PdOEP in degassed toluene with the following concentrations: BPEA (Absorbance: $5 \times 10^{-5} \mathrm{M}$,

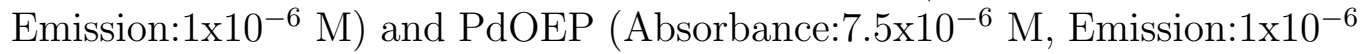
$\mathrm{M})$. 


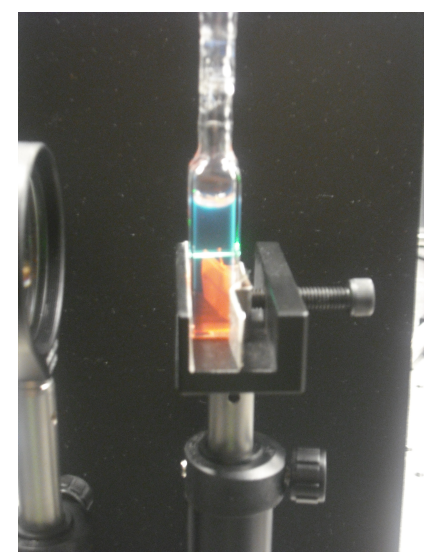

Fig. 6.6 Upconversion of BPEA $\left(10^{-3} \mathrm{M}\right)$ and $\operatorname{PdOEP}\left(10^{-4} \mathrm{M}\right)$ in a solution of degassed toluene.

The same solution of BPEA $\left(10^{-3} \mathrm{M}\right)$ and PdOEP $\left(10^{-4} \mathrm{M}\right)$ in toluene was used to determine if upconversion was occurring in solution. An excitation wavelength of $532 \mathrm{~nm}$ was used to directly excite the sensitizer. The resulting emission spectra of upconverted fluorescence was obtained Fig. 6.7) and no peak corresponding to phosphorescence was seen. This meant that though the sensitizer is directly excited, all phosphorescence is quickly quenched by BPEA and fluorescence of BPEA subsequently occurs. This is evidence that upconversion is occurring in this solution and that BPEA and PdOEP can be used to demonstrate upconversion. This is the first time that BPEA and PdOEP have been used together to demonstrate upconversion. 
78CHAPTER 6. SPECTROSCOPY OF DPA AND BPEA DERIVATIVES

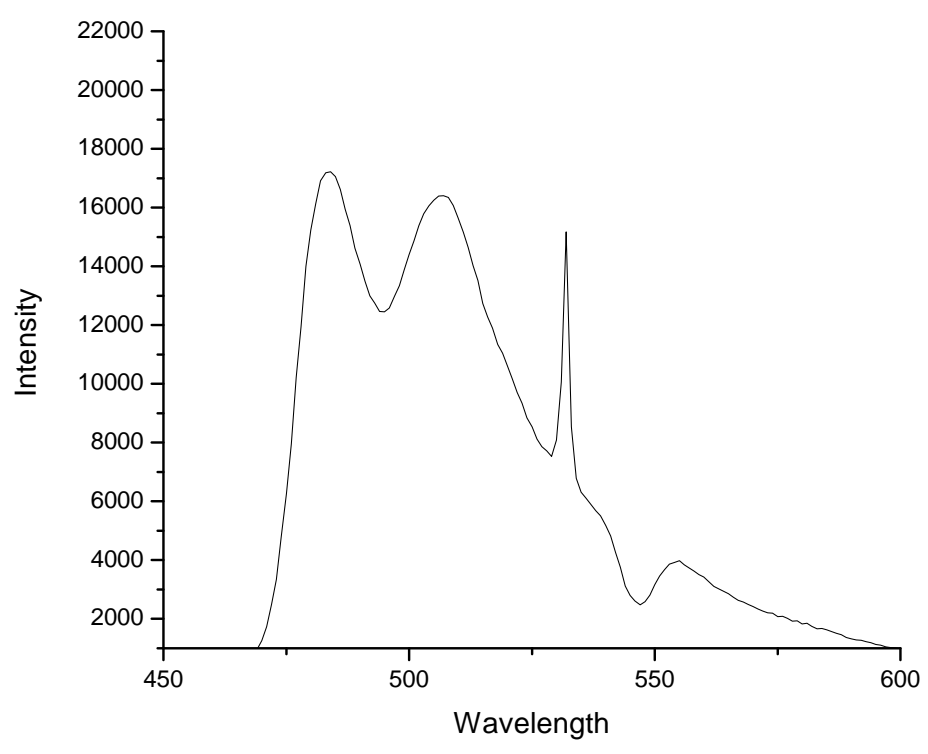

Fig. 6.7 Emission spectrum of a solution of BPEA $\left(10^{-3} \mathrm{M}\right)$ and PdOEP $\left(10^{-4} \mathrm{M}\right) \mathrm{PdOEP}$ solution in a degassed solution of toluene. 


\subsection{Spectroscopy of DPA Derivatives}

\subsubsection{Solution Phase Spectroscopy}

Absorption and emission spectra were obtained for each synthesized derivative in solution. The absorption spectra of the dicatenar derivatives do not vary from the original DPA dye, but are very slightly red shifted from that of DPA (Fig. 6.8). There are four main peaks in the absorption spectrum of DPA, 340, 355, 375 and $395 \mathrm{~nm}$. These peaks are shifted for the dicatenar derivatives. There are four main peaks 359, 377 and $397 \mathrm{~nm}$ however the highest energy peak becomes broadened and less defined.

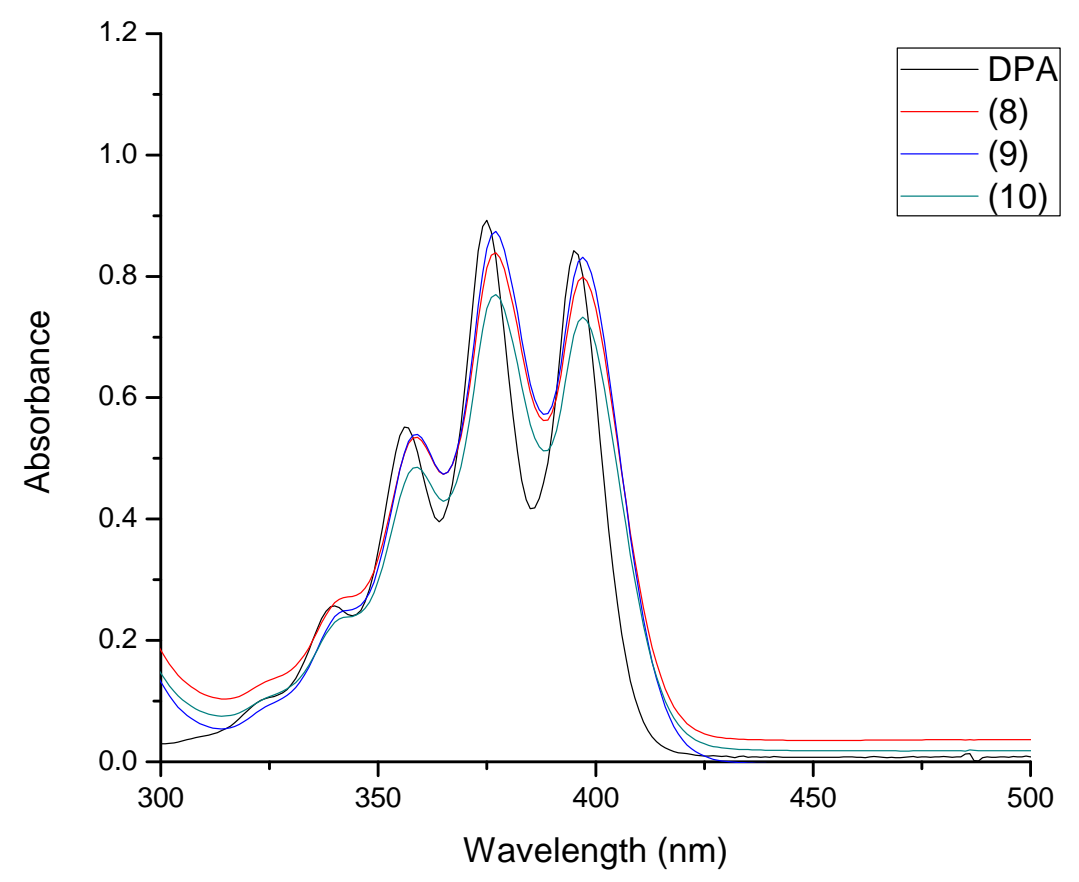

Fig. 6.8 Absorption spectra of dicatenar DPA derivatives $\left(7.5 \times 10^{-5} \mathrm{M}\right)$. 
80CHAPTER 6. SPECTROSCOPY OF DPA AND BPEA DERIVATIVES

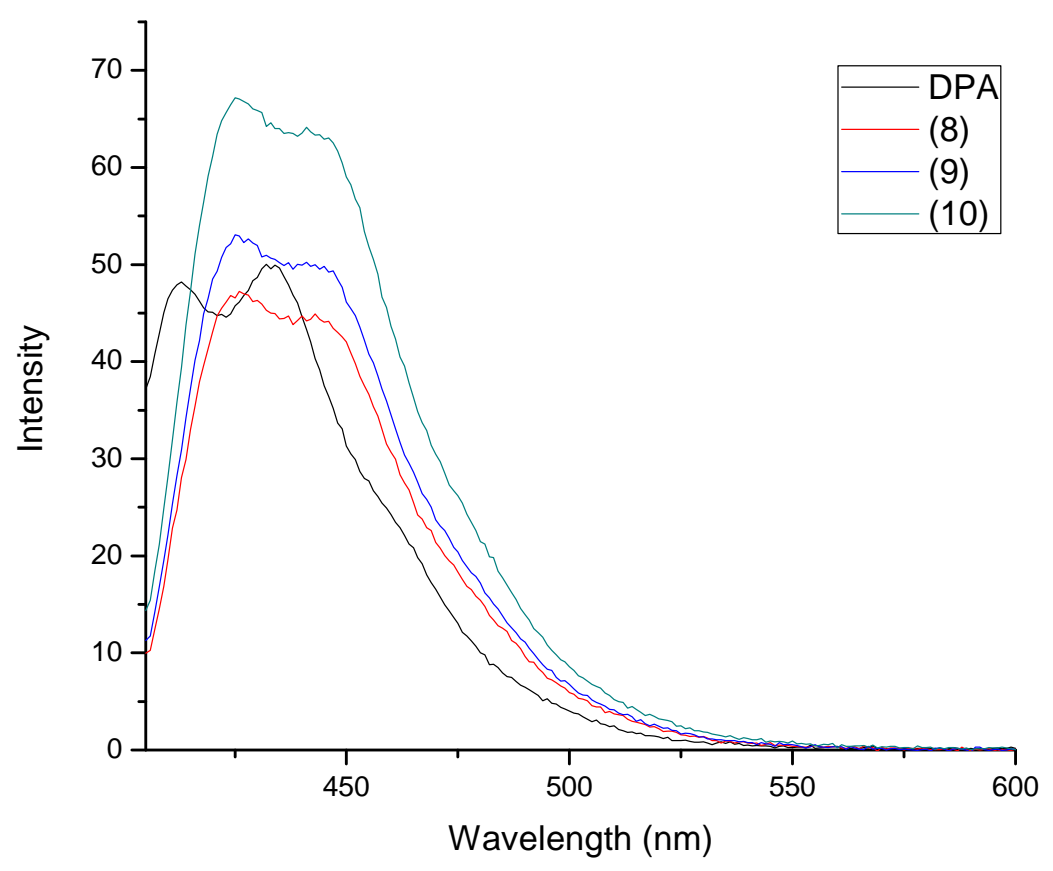

Fig. 6.9 Emission spectra of dicatenar DPA derivatives $\left(1 \times 10^{-6} \mathrm{M}\right)\left(\lambda_{e x}\right.$ $=395 \mathrm{~nm}$ ). 
The emission spectra of the dicatenar derivatives are also slightly redshifted (Fig. 6.9). The main peaks observed in the emission spectra of DPA are at 413 and $432 \mathrm{~nm}$. The mains peaks observed for fluorescence have shifted to 425 and $445 \mathrm{~nm}$. This red shift of the emission spectra of the dicatenar derivatives from the original DPA dye is more pronounced than the shift observed for the absorption spectra. In the emission spectrum of DPA it is the peak at $432 \mathrm{~nm}$ that is more intense than that at $413 \mathrm{~nm}$. The peak at 425 becomes more intense than that of $445 \mathrm{~nm}$ in the emission spectra of the dicatenar derivatives.

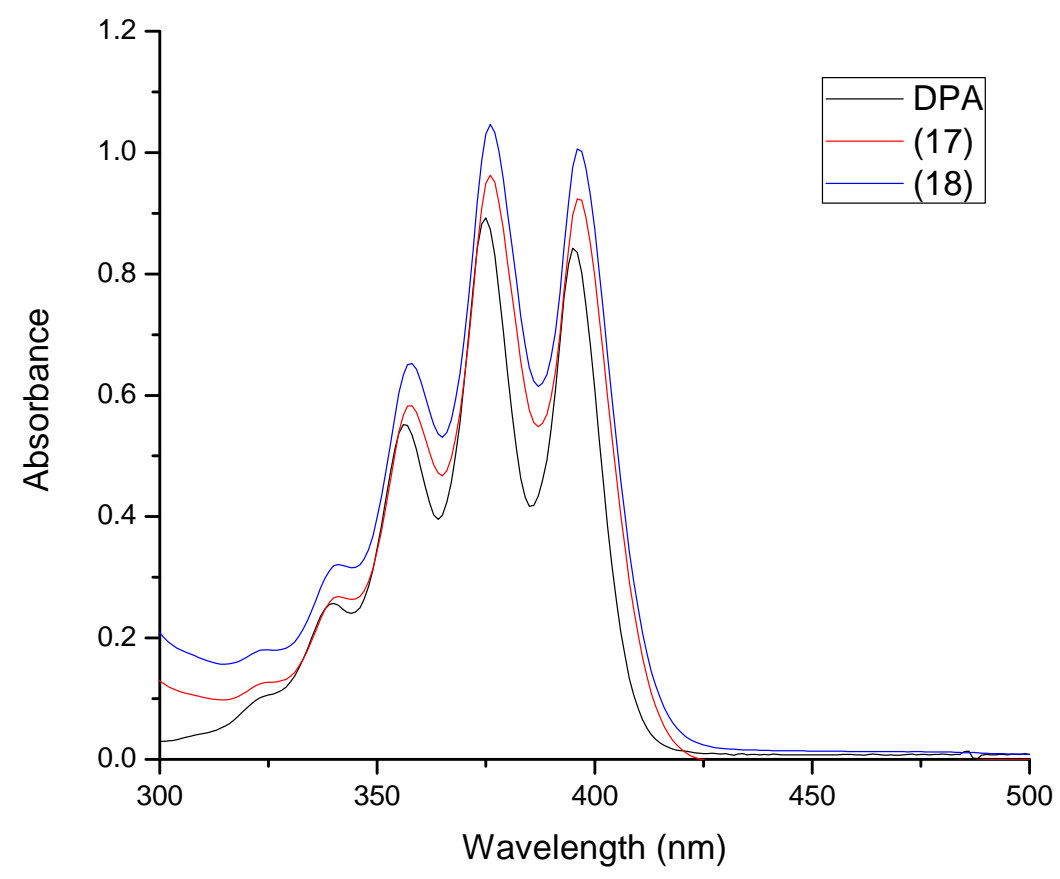

Fig. 6.10 Absorption spectra of hexacatenar DPA derivatives (7.5x10 $\mathrm{M})$.

The absorption spectra differ very little from the DPA absorption spectrum (Fig. 6.10). The main peaks observed are shifted by $1 \mathrm{~nm}$ to 358, 376, $396 \mathrm{~nm}$. The peaks imparted by the DPA core can no longer be clearly dis- 
tinguished in the fluorescence of the hexacatenar derivatives (Fig. 6.11). The two peaks are still red-shifted but have broadened into a single peak.

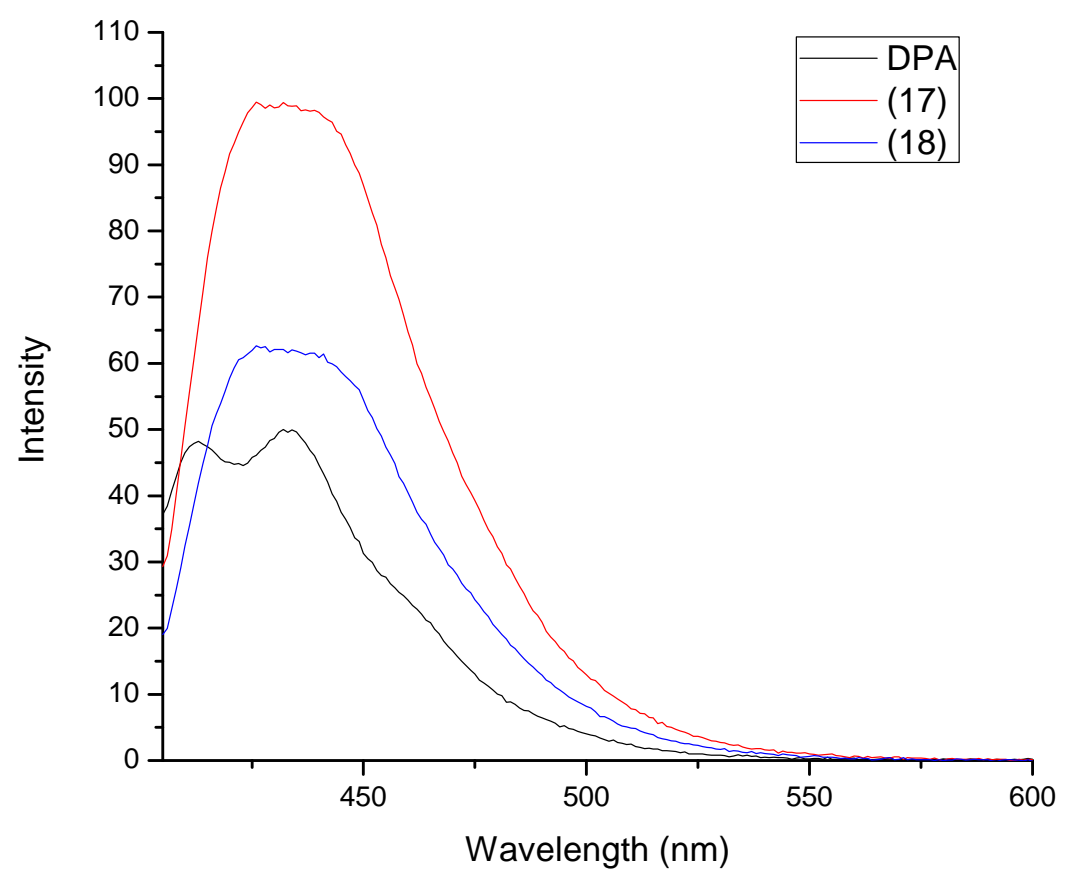

Fig. 6.11 Emission spectra of hexacatenar DPA derivatives $\left(1 \times 10^{-6} \mathrm{M}\right)$ $\left(\lambda_{e x}=395 \mathrm{~nm}\right)$.

\subsubsection{Hexacatenar Decyl DPA Derivative Thin Film Study}

Compound (17) exhibited extreme supercooling that allowed this material to remain in a neat liquid over several days. This neat liquid is one of a few derivatives of DPA that is a liquid at room temperature, one of which when blended with a sensitizer has resulted in upconversion.6768 This is because the neat liquid provides a condensed state in which all molecules are at close 
proximity in a liquid (defined by van der Waal's radii), at distances smaller than that of the Dexter radius which defines the distance for efficient electron transfer during upconversion. $\underline{5[6] 21}$

Absorption and emission spectra for (17) as a neat liquid thin film were obtained (Fig. 6.12) (Fig. 6.13). These were obtained by weighing some crystalline material of $(\mathbf{1 7})$ onto a glass slide which was melted into a neat thin liquid film. This process was repeated until a film of the appropriate absorption (absorbance below 0.2 ) was obtained $(0.3 \mathrm{mg}$ ). This film was further used in fluorescence measurements.

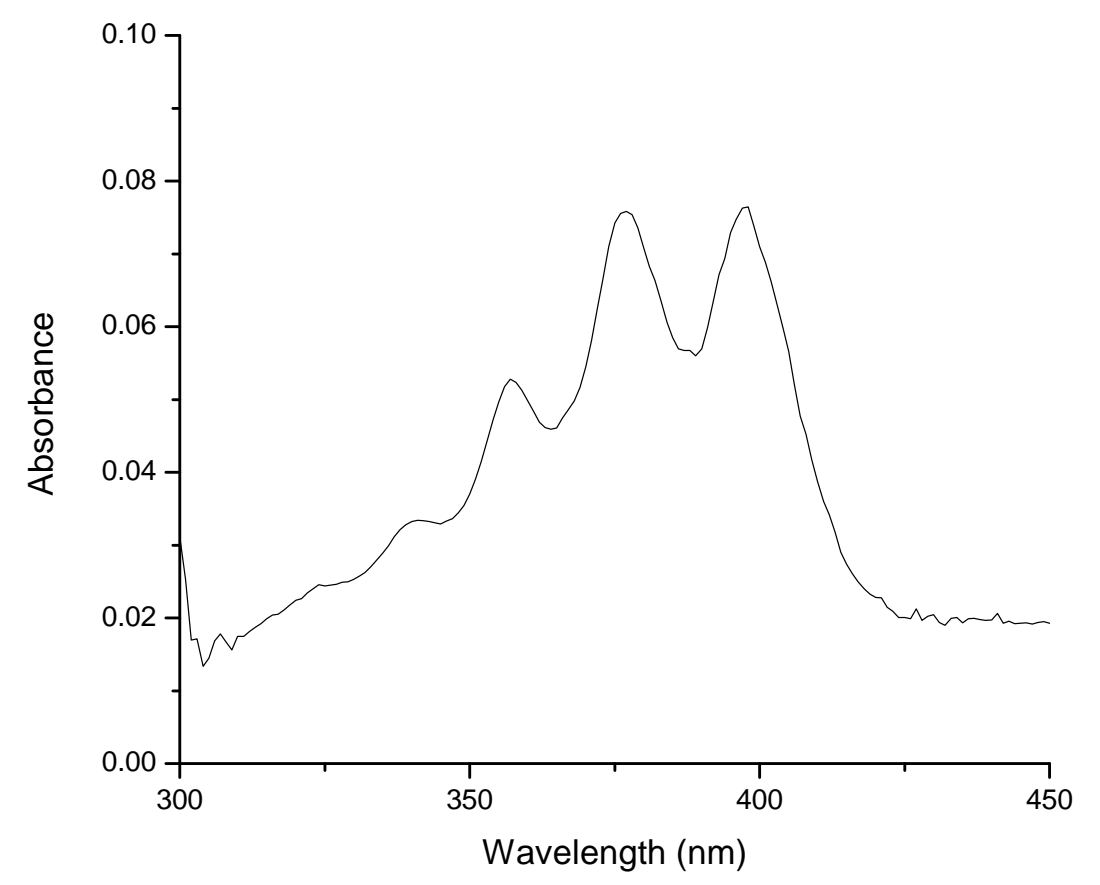

Fig. 6.12 Absorption spectra of (17) derivative in a thin film.

Absorbance of the film in its neat liquid state was able to be easily distinguished, and it varies very little from the absorption spectra of the solid state (Fig. 6.12). The main peaks of DPA at 340, 355, 375 and $395 \mathrm{~nm}$ have 
shifted in the neat liquid form of (17) to 341, 357, 377 and $398 \mathrm{~nm}$. This is noteworthy because it suggests minimal intermolecular electronic coupling in the condensed neat liquid, which is different from the large absorption change typically found when condensing from a solution to a solid.

Fluorescence of the same film was obtained using an excitation wavelength of $395 \mathrm{~nm}$ (Fig. 6.13), which bears much similarity to the emission of (17) in solution. The peaks cannot be clearly distinguished in the emission spectrum and one broad peak is observed. Absorbance of the crystalline solid was not able to be distinguished due to strong scattering of the crystalline solid and glass slide. These absorption and emission spectra for (17) vary very little from the absorption and emission in solution, which is very promising for a material for facilitation of upconversion and the same sensitizer PdOEP can still be used.

A thin neat liquid film for testing upconversion was prepared. A solution of the sensitzer PdOEP was dropcast onto a the neat liquid film so it was $2 \mathrm{wt}$ $\%$ in concentration. However, when it was placed in the optical set up with a green laser $\left(\lambda_{e x}=532 \mathrm{~nm}\right.$ ), so much scattering was present from the slide and excitation source that the upconverted fluorescence could not be detected. However, this is still a promising material for upconversion and future work should focus on varying the film thickness or amount of material present in the film which may make the upconverted fluorescence stronger and easier to detect. This would enable upconversion in this case to be further studied. 


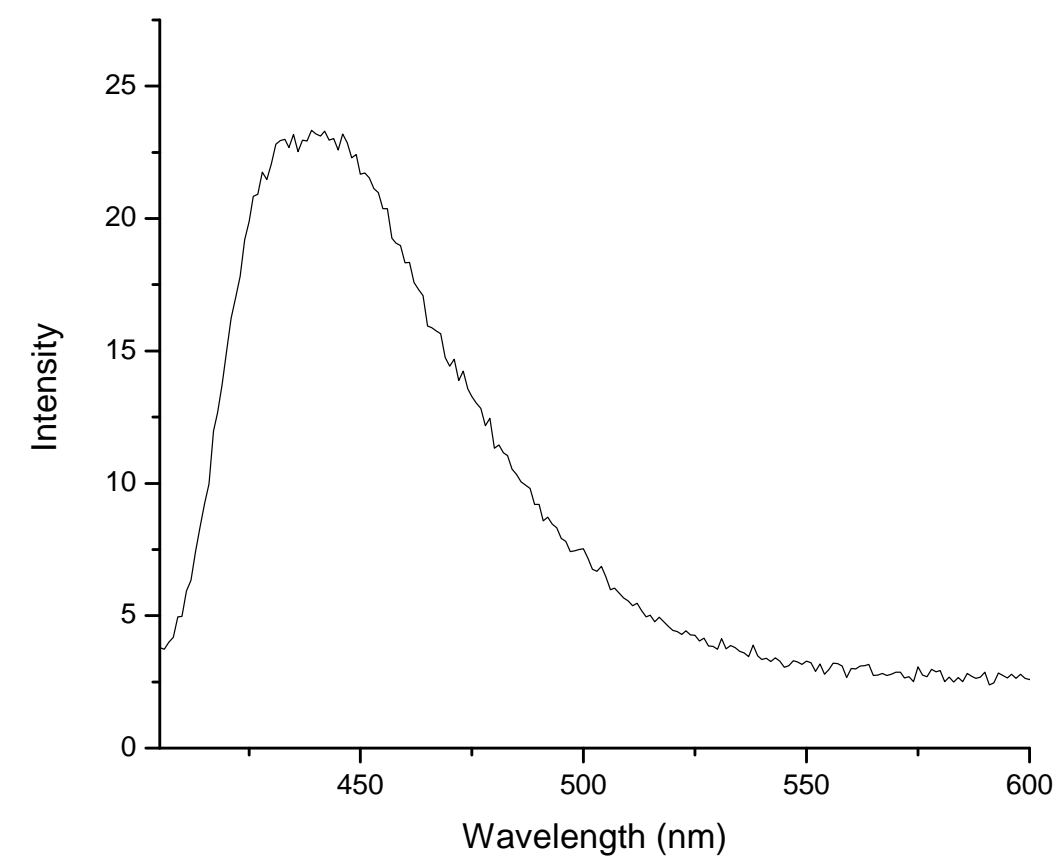

Fig. 6.13 Emission spectrum of (17) in a thin film $\left(\lambda_{e x}=395 \mathrm{~nm}\right)$. 


\title{
6.3 Spectroscopy of BPEA Derivatives
}

\subsubsection{Solution Phase Spectroscopy}

\begin{abstract}
Absorption and emission spectra were obtained for each synthesized derivative in solution. It should be noted that compound (22) was impure and this may alter the absorption and fluorescence spectra. The absorption spectra of the compounds (22) and (23) do not vary significantly from that of the original BPEA dye (Fig. 6.14). The BPEA dye has two vibronic peaks at 438 and $458 \mathrm{~nm}$. The absorption spectrum of $(\mathbf{2 3})$, has shifted to longer wavelengths (lower energy), the individual peaks can no longer be clearly distinguished and one broad peak is observed. The absorption spectrum of (24) is quite different compared with compounds $(\mathbf{2 2})$ and (23) and has the most redshifted absorption spectrum out of the three BPEA compounds synthesized. This is expected as the BPEA aromatic core has been further extended with phenyl substituents. It has two peaks which are the most facile to distinguish at 459 and $478 \mathrm{~nm}$. In contrast with the absorption spectrum of BPEA, the peak at $459 \mathrm{~nm}$ is the most intense.
\end{abstract}

In contrast to the absorption of the three snthesized BPEA derivatives, the emission spectra are more significantly red-shifted (Fig. 6.15). It should be noted that the excitation wavelength used was $400 \mathrm{~nm}$, as this is what is commonly used in the literature. ${ }^{[73}$ Compound (24) had the most intense fluorescence and was the most red-shifted out of the three synthesized derivatives. BPEA has two main vibronic peaks at 477 and $509 \mathrm{~nm}$, which are shifted to 492 and $524 \mathrm{~nm}$ for (23) and to 500 and $530 \mathrm{~nm}$ for (24). 


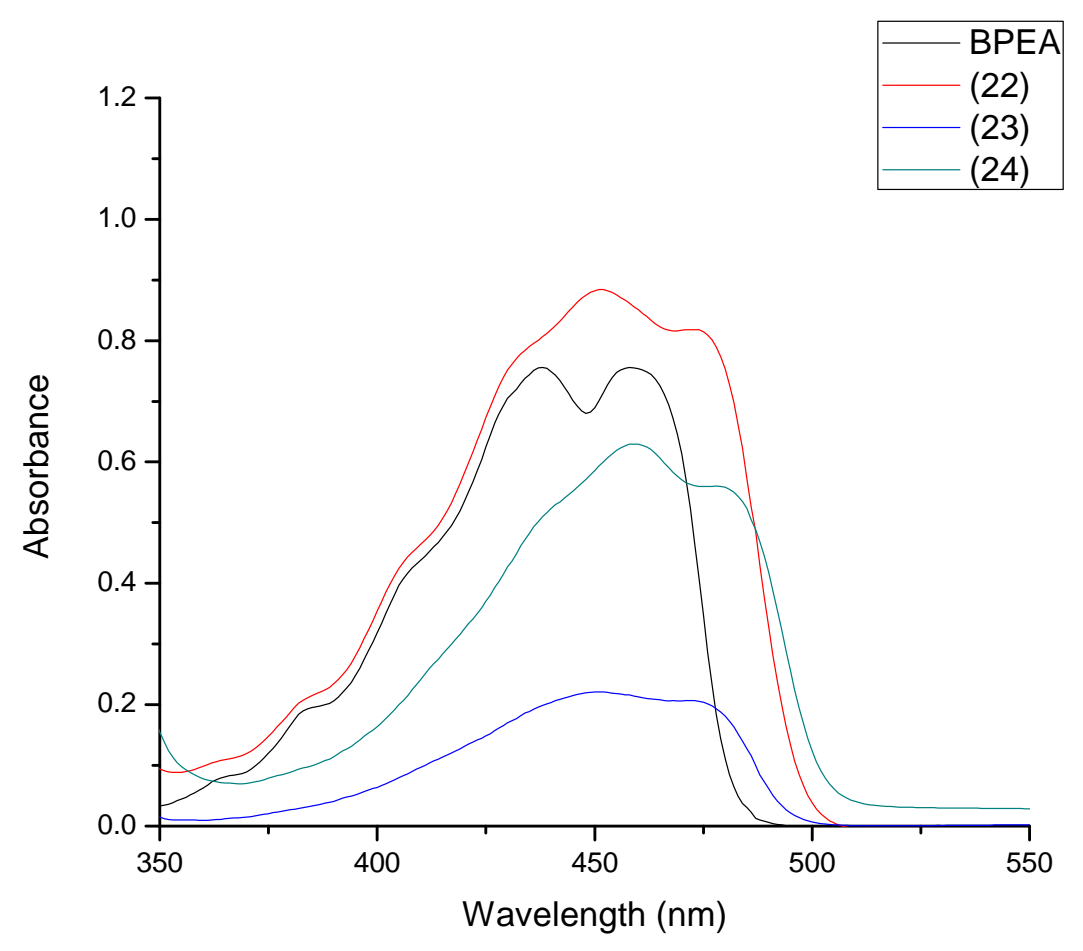

Fig. 6.14 Absorption spectra of BPEA derivatives $\left(5 \times 10^{-5} \mathrm{M}\right)$. 
88CHAPTER 6. SPECTROSCOPY OF DPA AND BPEA DERIVATIVES

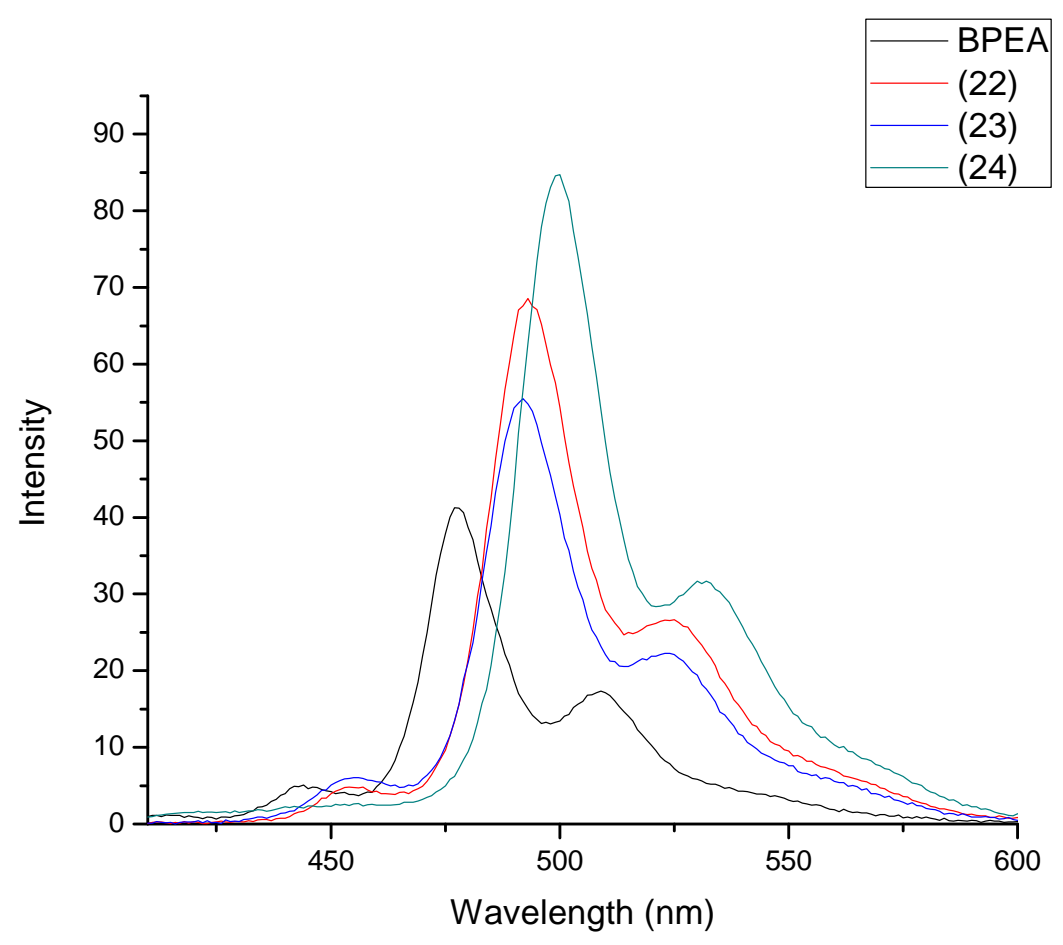

Fig. 6.15 Emission spectra of BPEA derivatives $\left(1 \times 10^{-6} \mathrm{M}\right)\left(\lambda_{e x}=400\right.$ $\mathrm{nm})$. 


\subsubsection{Thin Film Spectroscopy of the 9,10-Bis(biphenyl- 4-ylethynyl)anthracene (BBPEA) Derivative}

Compound (24) was investigated for its photophysical properties for its potential as an upconverting film. Absorption and emission spectra were obtained both at room temperature and at the temperature of the liquid crystal phase. A solution of $1 \mathrm{mgmL} L^{-1}(\mathbf{2 4})$ was drop cast in aliquots of $50 \mu \mathrm{L}$ onto a glass slide until a total of $200 \mu \mathrm{L}$ had been deposited. The film was heated to the temperature of the mesophase to yield a more uniform film and allowed to cool until crystallisation. Measurements in the liquid crystalline phase were obtained by heating the sample at a temperature slightly above the formation of the mesophase the liquid. Once this elevated temperature was attained, the slide was placed in the machine required and spectra taken as the sample cooled to room temperature.

Absorption spectra were obtained for (24) (Fig. 6.16). These spectra were also baseline corrected to try and eliminate the effects of scattering and variation between absorbance measurements of the thin film. In the absorption spectra of the liquid crystalline phase the individual peaks are lost and the absorption becomes broad. The larger peak at around $420 \mathrm{~nm}$ appears after 10-20 seconds, where the material reverts back to the crystalline state.

Emission spectra were also obtained for (24) (Fig. 6.17). In the crystalline thin film, there are well defined peaks around 550, 590 and $610 \mathrm{~nm}$. The peaks at 590 and $610 \mathrm{~nm}$ are reduced in intensity and broaden when the material enters the liquid crystalline phase. These spectra also indicated it took between 10 and 30 seconds for the material to crystallise.

This material was then blended with 2 wt\% sensitizer PdOEP. This was 


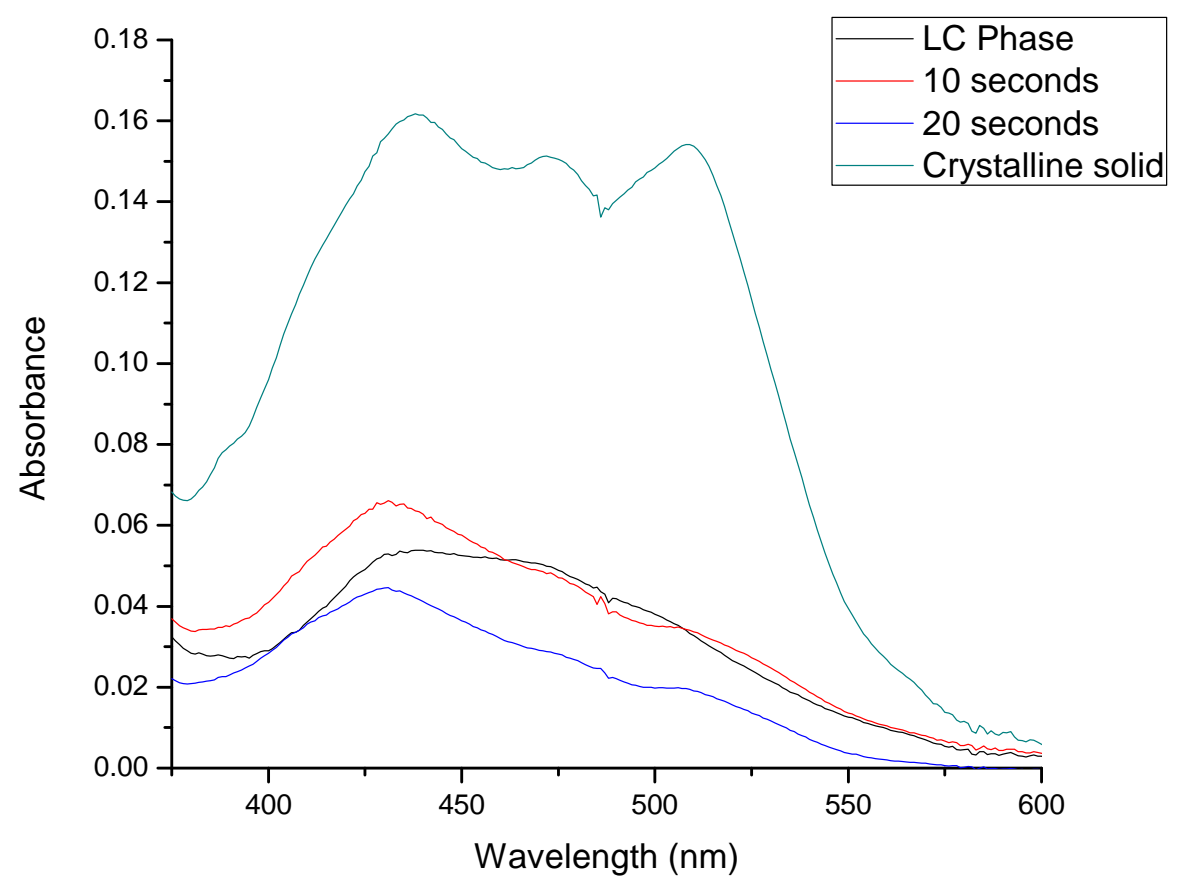

Fig. 6.16 Absorption spectra of (24) 


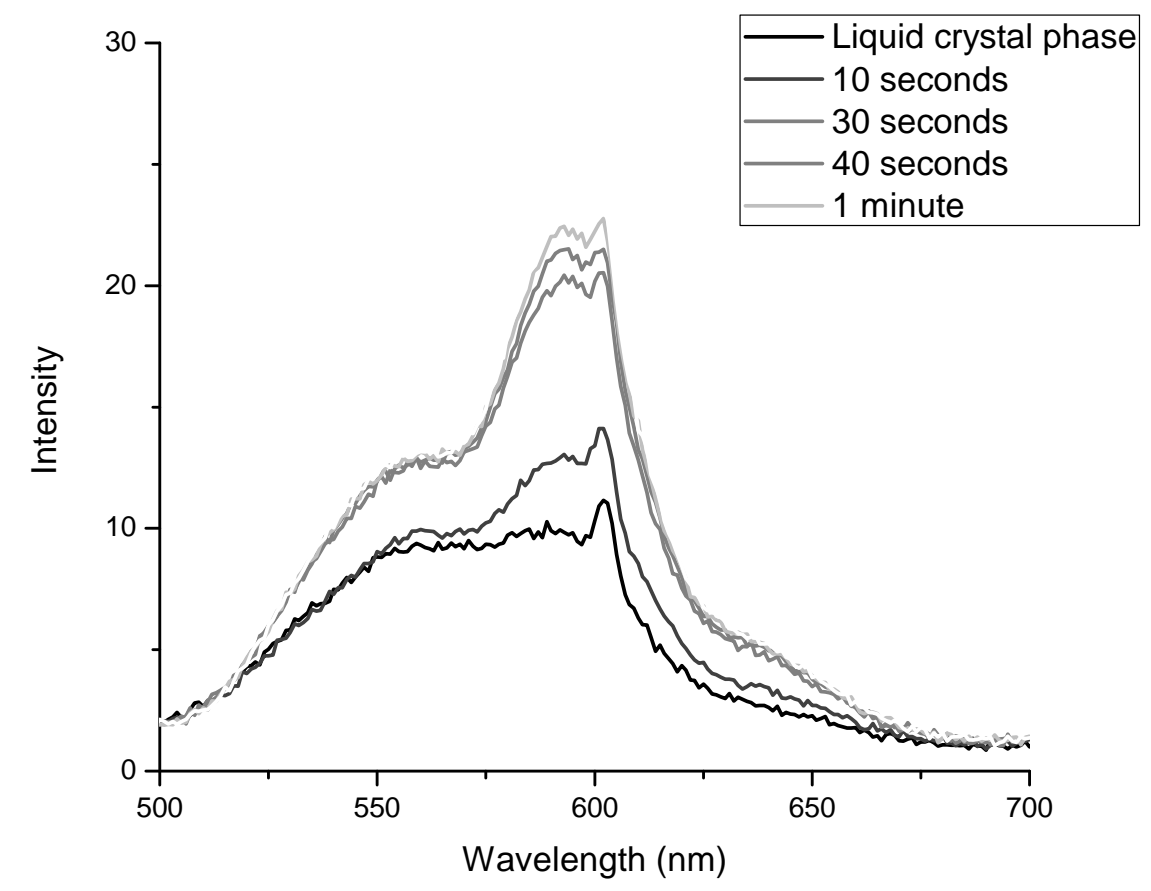

Fig. 6.17 Emission spectra of $(\mathbf{2 4})\left(\lambda_{e x}=400 \mathrm{~nm}\right)$. 
done to ascertain whether the liquid crystalline phase still formed in the presence of the sensitizer. The film was observed under the polarised optical microscope at a little above $209^{\circ} \mathrm{C}$, to ensure the sample was at the mesomorphic temperature. The liquid crystalline phase formed allowing needle shaped crystals of PdOEP to stay dispersed throughout the liquid (Fig. 6.18). Also, PdOEP did not decompose at this temperature. Both of these propertie are essential to a material that can facilitate upconversion.
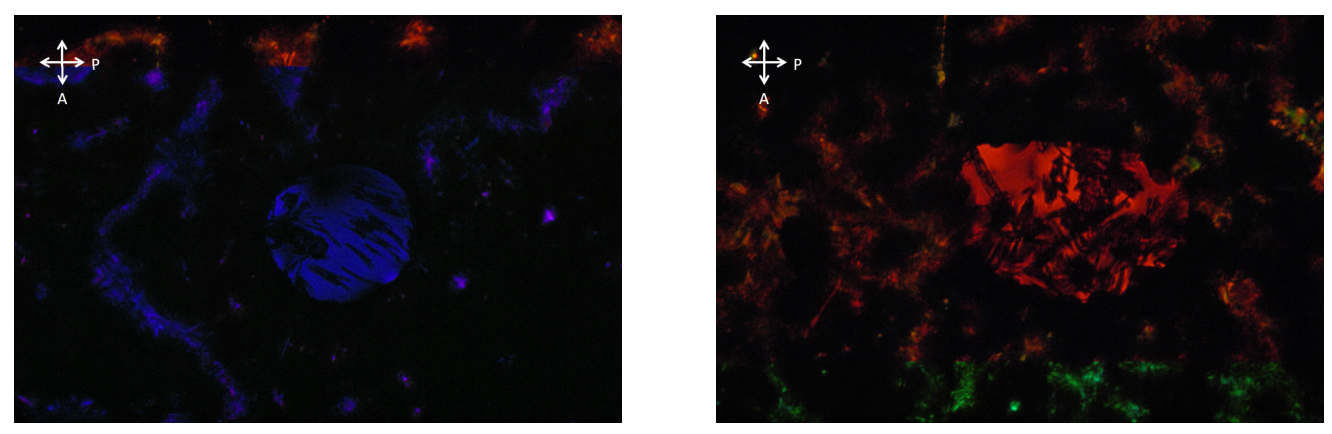

Fig. 6.18 POM Images of $(\mathbf{2 4})$ in a thin film blended with sensitizer at $215^{\circ} \mathrm{C}$ at $100 \mathrm{x}$ magnification.

Upconversion in this film was trialled. The available laser source used was a $532 \mathrm{~nm}$ green laser. In the crystalline state of $(\mathbf{2 4})$ the $532 \mathrm{~nm}$ overlaps with the absorption spectra, resulting in direct excitation of the emitter. The absorption undergoes a slight red-shift and broadens in the liquid crystalline phase. There was a possibility that the laser would not directly excite the emitter in the liquid crystalline phase, thus the experimental was still trialled.

The experiment resulted in fluorescence of the same kind being produced at both excitation of the crystalline solid and the liquid crystalline phase. This was assumed to be direct excitation of the emitter. The ultimate solution to this problem would be to use a different sensitizer such as mesotetraphenyl-tetrabenzoporphine palladium $\mathrm{PdPh}_{4} \mathrm{TBP}$ which has previously been used in conjunction with BPEA. $\frac{16] 17}{1}$ This emitter has an excitation wavelength of $635 \mathrm{~nm}$ which does not overlap with the absorption of the liquid crystalline or crystalline thin film of $(\mathbf{2 4})$. In this case the emitter would 
not be directly excited, and upconversion could be studied.

Also, it is clear the high phase temperature of this material creates large technological challenges in direct measurement of the sample. It is very tricky to maintain the correct temperature of the sample and work with the spectroscopic set up at the same time. This may alter the usability of this material for facilitating upconversion. A promising aspect of this material is that it retains its liquid crystal phase in the presence of the sensitizer, which means this material could be further tested to see if upconversion is possible.

\subsection{Conclusions}

The absorption and emission spectra for all derivatives synthesized in this thesis were obtained, and did not vary significantly from that of their dye derivatives. However, there were two derivatives that exhibited special properties that were further investigated in thin film form for potential use as a material for upconversion. The first material investigated was 9,10-bis(3,4,5tri(decyl) oxyphenyl)anthracene remained a liquid at room temperature over extended periods of time ( 3 days)and the second derivative investigated was 9,10-bis(4'-(octyloxy)biphenyl-4-ylethynyl)anthracene which exhibited mesophase formation at $209^{\circ} \mathrm{C}$. The novel decyl DPA derivative showed very little change in absorption or emission spectra than that of its DPA dye counterpart, which indicated that in the neat liquid state there was not significant amounts of electronic coupling. Upconversion may be exhibited if thicker thin film samples could be made. Palladium octaethylporphyrin was also blended with the liquid crystalline derivative in a thin film. The excitation source directly excited the emitter, and due to the high temperature of the phase there was difficulty in studying the upconversion process. The formation of the liquid crystal phase was retained on inclusion of the sensitizer also 
94CHAPTER 6. SPECTROSCOPY OF DPA AND BPEA DERIVATIVES

without the decomposition of the sensitizer. Upconversion could be properly studied with the inclusion of a more appropriate sensitizer such as $\mathrm{PdPh}_{4} \mathrm{TBP}$ and with appropriate methods for studying this at high temperatures. 


\section{Chapter 7}

\section{Conclusions and Future Work}

Overall, eight novel compounds have been synthesized in this thesis; five of which were DPA derivatives, and three BPEA derivatives. Not only have new syntheses of novel compounds been completed, but through analysis of the phase behaviour of these compounds new knowledge of what the requirements are for liquid crystalline behaviour using these central DPA and BPEA dye molecules have been gained.

Symmetrical direct substitution of these dyes does not result in liquid crystalline phases, as it favours the rigid ordering found in the crystalline solid. It has been shown in the literature that asymmetrical alkyl substitution as well as the addition of at least one phenyl ring does result in liquid crystalline compounds. If direct substitution of the central core is needed, future work should focus on establishing efficient syntheses of these derivatives, as they currently rely on the production of statistical mixtures. Direct substitution of the anthracene ring did result in a hexacatenar decyl derivative with interesting properties. It has been found to undergo extreme supercooling, so that the substance will remain as a liquid at room temperature for 
several days before eventual crystallisation. There are only a few examples of DPA liquids at room temperature and is the first to undergo such supercooling. This material also shows promise as an upconversion material. The photophysical properties have not been significantly altered from that of the original DPA core and in the neat liquid state molecules are at close proximity (defined by van der Waals' radii) within the Dexter radius which defines the distance on which upconversion can occur effectively. On inclusion of a sensitizer it would be expected that upconversion could occur given the achievement upconversion with similar derivatives.

On inclusion of the sensitizer into a neat liquid film the upconverted fluorescence was not strong enough to be detected. Thus, future work will need to focus on forming thicker films with more material present and optimisation of a spectroscopic set up in order to compare the efficiency.

A liquid crystalline derivative, 9,10-bis(4'-(octyl)oxybiphenyl-4-ylethynyl) anthracene has been synthesized. It formed a mesophase at elevated temperatures. The liquid crystalline derivative was blended in with the sensitzer, where it was found that the liquid crystalline phase was retained. The sensitizer did not decompose but was dispersed throughout the liquid crystalline phase. However it was found that in both crystalline and liquid crystalline states that the excitation wavelength used to excite the sample overlapped with the absorption spectrum of the emitter and resulted in direct excitation of the emitter molecule. Future work using a more appropriate sensitizer such as meso-tetraphenyl-tetrabenzoporphine palladium $\left(\mathrm{PdPh}_{4} \mathrm{TBP}\right)$ would resolve this, and allow the upconversion process and efficiency of this material to be properly investigated.

The work of this thesis has resulted in the discovery of novel materials, which with further testing may be able to facilitate and enhance the process of upconversion. 


\section{Chapter 8}

\section{Experimental}

\subsection{Synthetic Methods}

All chemicals were obtained from Sigma-Aldrich or Merck and used as recieved unless otherwise stated. The following compounds were synthesized and supplied by other group members using literature techniques: tri-tertbutylphosphonium tetrafluoroborate, tris(dibenzylideneacetone)dipalladium. Trimethylborate was distilled to remove the azeotrope from solution. The solvents for reactions were of analytical grade and used as recieved, except for tetrahydrofuran and dichloromethane which were degassed and stored over molecular sieves. All Suzuki, Sonogashira and Williamson ether syntheses were performed under inert conditions. NMR spectra were obtained in deuterated chloroform $\left(\mathrm{CDCl}_{3}\right)$ (Sigma-Aldrich, $99.8 \%$ atom D, stored over $4 \AA$ molecular sieves) in a Varian Unity Inova 500 spectrometer with ${ }^{1} \mathrm{H}$ resonance at $500 \mathrm{MHz}$ and ${ }^{13} \mathrm{C}$ at $125 \mathrm{MHz}$. In all other measurements common laboratory practice was followed. 9,10-Bis(4'-(octyloxy)biphenyl-4ylethynyl)anthracene and 9,10-bis(4-(octyloxy)phenylethynyl)anthracene were 
synthesized and supplied by Dr. K. Allan, for which only the NMR data for each compound will be presented below. .69

\subsubsection{Synthesis of Dicatenar DPA Derivatives}

4-Methoxyphenyl boronic acid (4). Tetrahydrofuran (50 mL) was added to an excess of magnesium turnings $(2.43 \mathrm{~g}, 100 \mathrm{mmol}$ ) (stirred overnight under nitrogen). 4-Bromoanisole (12.50 mL, $99 \mathrm{mmol}$ ) was added dropwise to this solution and the flask was placed in an ice bath. The reaction mixture remained in the bath until the flask had reached a steady temperature. This Grignard solution and trimethyl borate $(11.34 \mathrm{~mL}, 100 \mathrm{mmol})$ were added alternately in small quantities to a cooled flask $\left(-60^{\circ} \mathrm{C}\right)$ charged with dry diethyl ether $(40 \mathrm{~mL})$. The reaction mixture was allowed to warm to room temperature and stirred for a further 20 minutes. The flask was cooled to $0{ }^{\circ} \mathrm{C}$ and the reaction hydrolysed by the addition of water $(6.60 \mathrm{~mL})$ over 5 minutes. The reaction was neutralized by the addition of concentrated sulfuric acid over 15 minutes. The resulting organic layer was separated and washed with water. This layer was dried over anhydrous magnesium sulfate, filtered solvent removed in vacuo. An off-white solid was obtained which was used without further purification. (Yield: $8.05 \mathrm{~g}, 40 \%$ )

9,10-Dibromoanthracene (5). A solution of bromine $(5.8 \mathrm{~mL}, 225$ $\mathrm{mmol})$ in acetic acid $(50 \mathrm{~mL})$ was added dropwise over 5 minutes to a stirred suspension of anthracene $(10 \mathrm{~g}, 56 \mathrm{mmol})$ in acetic acid $(300 \mathrm{~mL})$. The reaction mixture was stirred for 30 minutes. A three necked $500 \mathrm{~mL}$ flask used in this reaction was equipped with an overhead stirrer, equalized dropping funnel and gas trap (using a solution of sodium hydroxide to neutralise evolved hydrobromic acid). The resulting mixture was poured into water $(300 \mathrm{~mL})$ resulting in precipitation of a yellow solid. This was filtered, washed with water and dried in vacuo. Yellow platelike crystals were obtained and used 
without further purification. (Yield: $16.98 \mathrm{~g}, 90 \%$ )

9,10-Dianisylanthracene (6). Tris(dibenzylideneacetone)dipalladium(0) $(0.06 \mathrm{~g}, 0.07 \mathrm{mmol}), 4(1.26 \mathrm{~g}, 8 \mathrm{mmol}), 5$ (1.13 g, $3.36 \mathrm{mmol})$, tri-tertbutylphosphonium tetrafluoroborate $(0.04 \mathrm{~g}, 0.14 \mathrm{mmol})$, and potassium fluoride $(1.45 \mathrm{~g}, 24 \mathrm{mmol})$ were transferred to a Schlenk tube. Dry tetrahydrofuran $(12 \mathrm{~mL})$ was added and the resulting mixture stirred vigorously for 2 hours. This reaction was quenched by addition of diethylether (40 $\mathrm{mL}$ ). The solvent was removed in vacuo to give a pale yellow crude product. The product was obtained through purification of the crude through Soxhlet extraction. (Yield: $1.22 \mathrm{~g}, 98 \%$ )

9,10-Bis(4-hydroxyphenyl)anthracene (7). 33 wt \% Hydrobromic acid in acetic acid $(20 \mathrm{~mL}, 121.5 \mathrm{mmol})$ was added dropwise to 6 (1.06 g, $2.7 \mathrm{mmol}$ ). The mixture was stirred and heated at reflux for $48 \mathrm{hrs}$. The resulting mixture was poured into an excess of distilled water and filtered through a silica frit. A hygroscopic green solid was obtained and dried in vacuo. (Yield: $0.97 \mathrm{~g}, 99 \%$ )

9,10-Bis(4-(octyloxy)phenyl)anthracene (8). 1-Bromooctane (1.03 $\mathrm{mL}, 6 \mathrm{mmol})$ was added to a stirred solution of 7 (0.51 g, $1.4 \mathrm{mmol})$, potassium carbonate $(0.69 \mathrm{~g}, 5 \mathrm{mmol})$ and acetonitrile $(30 \mathrm{~mL})$. The mixture was allowed to react at reflux for 4 days. The product was extracted with chloroform, and washed with water. The resulting organic layer was dried over anhydrous magnesium sulfate, filtered and dried in vacuo. The resulting product was recrystallised from methanol. (Yield: $0.35 \mathrm{~g}, 42.42 \%{ }^{1} \mathrm{H} \mathrm{NMR}$ $\delta\left(500 \mathrm{MHz} \mathrm{CDCl}_{3}\right): 7.75(\mathrm{~m}, 4 \mathrm{H}, \mathrm{H} 1), 7.38$ (d, J = $\left.8 \mathrm{~Hz}, 4 \mathrm{H}, \mathrm{H} 6\right), 7.33$ (m, $4 \mathrm{H}, \mathrm{H} 2), 7.13$ (d, 4H, J = $7 \mathrm{~Hz}, \mathrm{H} 7), 4.11$ (t, J = $5 \mathrm{~Hz}, 4 \mathrm{H}, \mathrm{H} 9), 1.89$ (m, 4H, H10-H15), 1.55 (m, 4H, H10-H15), 1.5-1.2 (m, 16H, H10-H15), 0.92 (m, 6H, H16). Selected ${ }^{13} \mathrm{C}$ NMR $\delta\left(125 \mathrm{MHz}, \mathrm{CDCl}_{3}\right.$ ): 132.30 (s, C6), 125.19 (s, C2), 127.11 (s, C1), 114.36 (s, C7), 68.30 (s, C9), 26.11-31.87 (s, C10-C15), 
14.18 (s, C16).

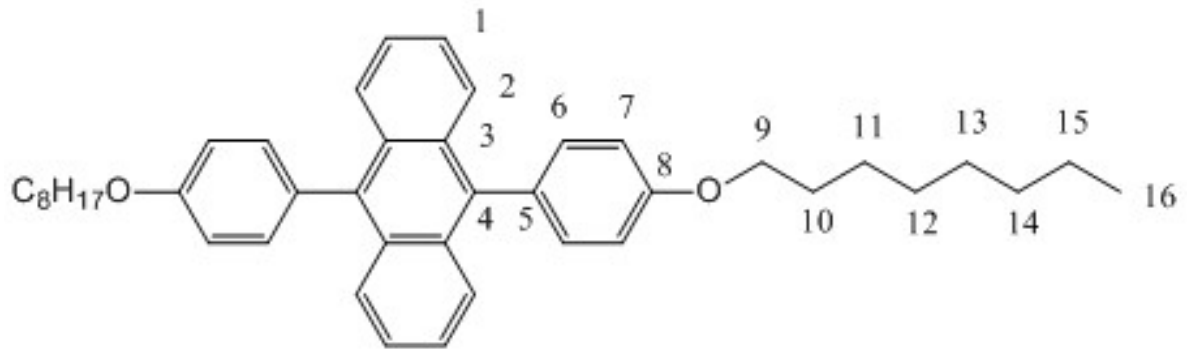

9,10-Bis(4-(dodecyloxy)phenyl)anthracene (9). 1-Bromododecane $(0.3 \mathrm{~mL}, 1.2 \mathrm{mmol})$ was added to a stirred solution of $7(0.1 \mathrm{~g}, 0.27 \mathrm{mmol})$, potassium carbonate $(0.14 \mathrm{~g}, 1 \mathrm{mmol})$ and acetonitrile $(6 \mathrm{~mL})$. The mixture was allowed to react at reflux for 4 days. The product was filtered through a silica frit and washed with small portions of water and hexane. The resulting powder was recrystallised from a mixture of chloroform/methanol and dried in vacuo. (Yield: $0.0645 \mathrm{~g}, 33 \%){ }^{1} \mathrm{H} \mathrm{NMR} \delta\left(500 \mathrm{MHz}, \mathrm{CDCl}_{3}\right): 7.75(\mathrm{~m}$, $4 \mathrm{H}, \mathrm{H} 1), 7.38$ (d, J = 8.5 Hz, 4H, H6), 7.33 (m, 4H, H2), 7.13 (d, 4H, J = $9 \mathrm{~Hz}, \mathrm{H} 7), 4.11$ (t, J = 6.5 Hz, 4H, H9), 1.89 (m, 4H, H10-H19), 1.55 (m, $4 \mathrm{H}, \mathrm{H} 10-\mathrm{H} 19)$, 1.5-1.2 (m, 16H, H10-H19), 0.92 (m, 6H, H20). Selected ${ }^{13} \mathrm{C}$ NMR $\delta\left(125 \mathrm{MHz}, \mathrm{CDCl}_{3}\right): 132.36$ (s, C6), 124.81 (s, C2), 127.11 (s, C1), 114.37 (s, C7), 68.21 (s, C9), 26.16-31.87 (s, C10-C19), 14.15 (s, C20).

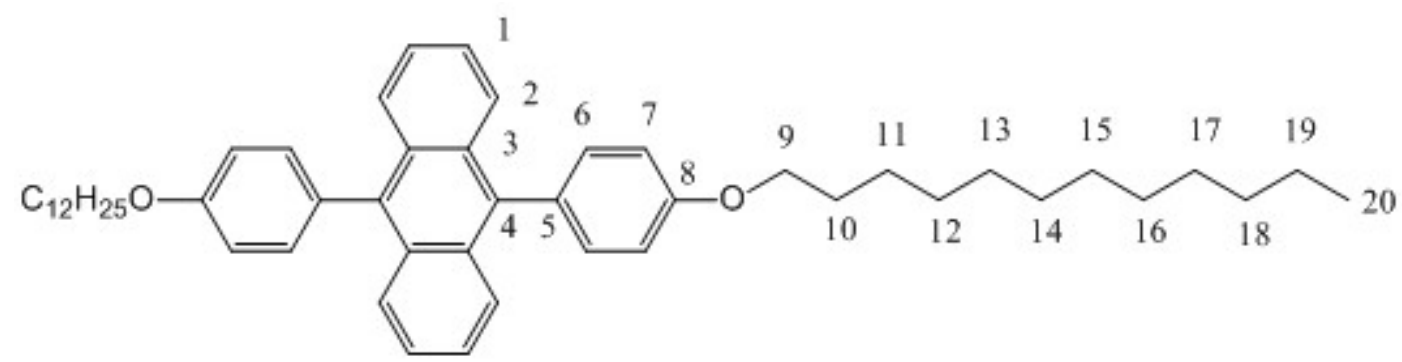


9,10-Bis(4-(hexadecyloxy)phenyl)anthracene (10). 1-Bromohexadecane $(0.4 \mathrm{~mL}, 1.2 \mathrm{mmol})$ was added to a stirred solution of $7(0.1 \mathrm{~g}, 0.27 \mathrm{mmol})$, potassium carbonate $(0.14 \mathrm{~g}, 1 \mathrm{mmol})$ and acetonitrile $(6 \mathrm{~mL})$. The mixture was allowed to react at reflux for 4 days. The product was filtered through a silica frit, washed with small portions of water and hexane. The resulting product was recrystallised from a mixture of chloroform/methanol and dried under vacuum. (Yield: $0.051 \mathrm{~g}, 22.18 \%) .{ }^{1} \mathrm{H}$ NMR $\delta\left(500 \mathrm{MHz}, \mathrm{CDCl}_{3}\right.$ ): 7.75 (m, 4H, H1), 7.38 (d, J = 8 Hz, 4H, H6), 7.33 (m, 4H, H2), 7.13 (d, 4H, $\mathrm{J}=8 \mathrm{~Hz}, \mathrm{H} 7), 4.11$ (t, J = 6.5 Hz, 4H, H9), 1.89 (q, 4H, H10-H23), 1.57 (m, 4H, H10-H23), 1.5-1.2 (m, 16H, H10-H23), 0.92 (m, 6H, H24). Selected ${ }^{13} \mathrm{C}$ NMR $\delta\left(125 \mathrm{MHz}, \mathrm{CDCl}_{3}\right): 132.72$ (s, C6), 125.13 (s, C2), 127.45 (s, C1), 114.58 (s, C7), 68.41 (s, C9), 26.57-30.08 (s, C10-C23), 14.15 (s, C24).

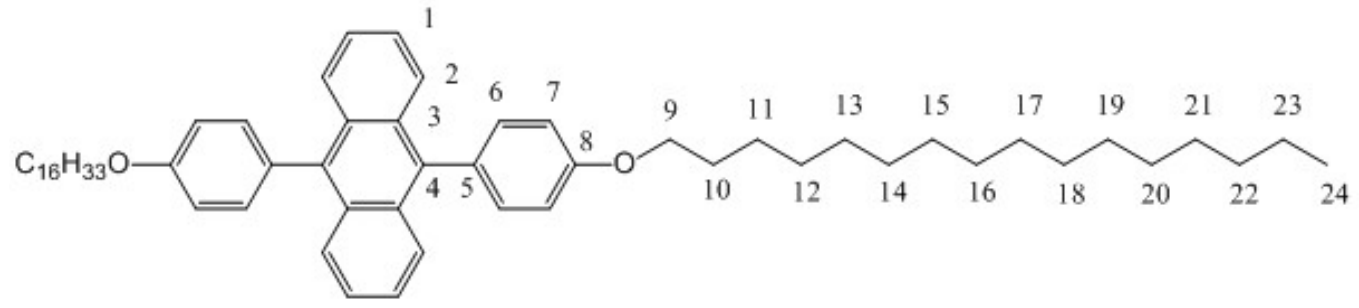

\subsubsection{Synthesis of Hexacatenar DPA Derivatives}

3,4,5-Trimethoxyphenylboronic acid pinacol ester (14). $1.6 \mathrm{M} \mathrm{n}$ butyllithium in hexane $(15 \mathrm{~mL}, 0.024 \mathrm{~mol})$ was added dropwise to a cooled flask $\left(-78^{\circ} \mathrm{C}\right)$ of 1 -Bromo-3,4,5-trimethoxybenzene $(5 \mathrm{~g}, 0.02 \mathrm{~mol})$ in dry tetrahydrofuran $(250 \mathrm{~mL})$. The mixture was allowed to react for 1 hour while the flask warmed to room temperature. 2-isopropoxy-4,4,5,5-tetramethyl1,3,2-dioxaborolane (9.06 mL, $0.044 \mathrm{~mol}$ ) was added and the mixture allowed to react overnight. The mixture was poured into a large amount of water 
and extracted with ethylacetate. The resulting organic layer was dried over anhydrous magnesium sulfate, filtered and the solvent removed in vacuo. (Yield: $4.46 \mathrm{~g}, 99 \%$ )

9,10-Bis (3,4,5-trimethoxyphenyl)anthracene (15). Tris (dibenzylideneacetone) dipalladium(0) (0.22 g, $0.24 \mathrm{mmol})$, 14 (3.96 g, $13 \mathrm{mmol})$, 5 (2.82 g, $8.4 \mathrm{mmol})$, tri-tert-butylphosphonium tetrafluoroborate $(0.28 \mathrm{~g}$, $1 \mathrm{mmol})$, and potassium fluoride $(2.92 \mathrm{~g}, 50 \mathrm{mmol})$ were transferred to a Schlenk tube. Dry tetrahydrofuran $(65 \mathrm{~mL})$ was added and the resulting mixture stirred vigorously at $50^{\circ} \mathrm{C}$ and the mixture left to react for 5 days. Solvent was removed in vacuo and the resulting crude powder extracted with water and chloroform. The organic layer was dried over anhydrous magnesium sulfate, filtered and solvent removed in vacuo. A yellow powder was obtained which was further purified through column chromatography on silica using a solvent mix of $25 \%$ acetone in hexanes to yield the product. (Yield: $2.2 \mathrm{~g}, 51.3 \%)^{1} \mathrm{H}$ NMR $\delta\left(500 \mathrm{MHz} \mathrm{CDCl}_{3}\right): 7.8$ (m, 4H, H2), 7.4 (m, 4H, H1), 6.7 (s, 4H, H6), 4.0 (s, 6H, H10), 3.85 (s, 12H, H8). ${ }^{13} \mathrm{C}$ NMR $\delta\left(125 \mathrm{MHz}, \mathrm{CDCl}_{3}\right): 153.5$ (s, C7), 137.6 (s, C9), 137.2 (2 singlets, C3 and C4), 134.5 (s, C5), 127 (s, C2), 125.5 (s, C1), 108.27 (s, C6), 61.1 (s, C10), 56.3 ( $\mathrm{s}, \mathrm{C} 8)$.

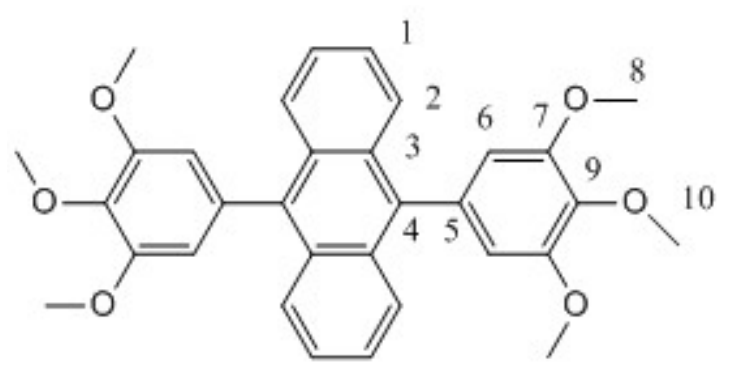

The syntheses of 16, 17 and $\mathbf{1 8}$ were carried out at the University of York in the United Kingdom by Professor John Spencer. Sufficient quantities of boron tribromide (as were required for this step) were available which could 
not be acquired when required in New Zealand. All materials were supplied by Sigma-Aldrich unless otherwise stated.

9,10-Bis(3,4,5-trihydroxyphenyl)anthracene (16). 15 (0.29 g, 0.57 $\mathrm{mmol}$ ) was dissolved in dry dichloromethane in a Schlenk tube under nitrogen, and the resulting solution cooled to $0^{\circ} \mathrm{C}$. A solution of boron tribromide in dichloromethane $(5 \mathrm{~mL}, 1 \mathrm{M}, 5 \mathrm{mmol})$ was added dropwise over $10 \mathrm{~min}$, resulting in the formation of clear dark yellow-brown solution after $30 \mathrm{~min}$. This solution was left to react for $2.5 \mathrm{hrs}$. Volatiles were removed in vacuo. The crude material was washed with water $(2 \times 3 \mathrm{~mL})$ and thoroughly dried in vacuo with gentle heating. This material was used without further purification.

9,10-Bis(3,4,5-tri(decyloxy)phenyl)anthracene (17). 16 was then suspended in dry dimethylformamide $(10 \mathrm{~mL})$ under nitrogen and potassium carbonate $(0.6 \mathrm{~g}, 4.34 \mathrm{mmol})$, bromodecane $(0.75 \mathrm{~mL}, 3.61 \mathrm{mmol})$ and dibenzo-18-crown-6 (0.002 g, $0.005 \mathrm{mmol})$ were added. The mixture was stirred and allowed to react at $70^{\circ} \mathrm{C}$ for $18 \mathrm{~h}$. After cooling, the mixture was treated with water $(20 \mathrm{~mL})$, and the mixture extracted three times with dichloromethane $(20 \mathrm{~mL})$. The combined organic fractions were dried over anhydrous magnesium sulfate and solvent removed in vacuo leaving a brown oil that was further dried in vacuo. The crude material was purified by column chromatography on silica, eluting first with petroleum ether and progressively increasing the polarity of the eluent. A very pale yellow fraction eluted with almost pure acetone. This afforded $0.02 \mathrm{~g}$ pale yellow crystals of $\mathbf{1 7}$ on recrystallization from chloroform/ethanol. Further crude product $(0.057 \mathrm{~g})$ was also recovered from the next column fraction but this was contaminated with a highly coloured impurity. (Yield: $0.02 \mathrm{~g}$ (pure material) + $0.057 \mathrm{~g}$ (crude), $10.7 \%){ }^{1} \mathrm{H}$ NMR $\delta\left(500 \mathrm{MHz} \mathrm{CDCl}_{3}\right): 7.79$ (m, 4H, H1), $7.36(\mathrm{~m}, 4 \mathrm{H}, \mathrm{H} 2), 6.64(\mathrm{~s}, 4 \mathrm{H}, \mathrm{H} 6), 4.13(\mathrm{t}, \mathrm{J}=5 \mathrm{~Hz}, 4 \mathrm{H}, \mathrm{H} 19), 3.94$ (t, J $=10 \mathrm{~Hz}, 8 \mathrm{H}, \mathrm{H} 8), 1.89$ (q, 4H, H20), 1.79 (q, 8H, H9), 1.57-1.2 (m, 84H, 
H10-H16 and H20-H27), 0.87 (m, 18H, H17 and H28). For carbon NMR see hexadecyl derivative below.

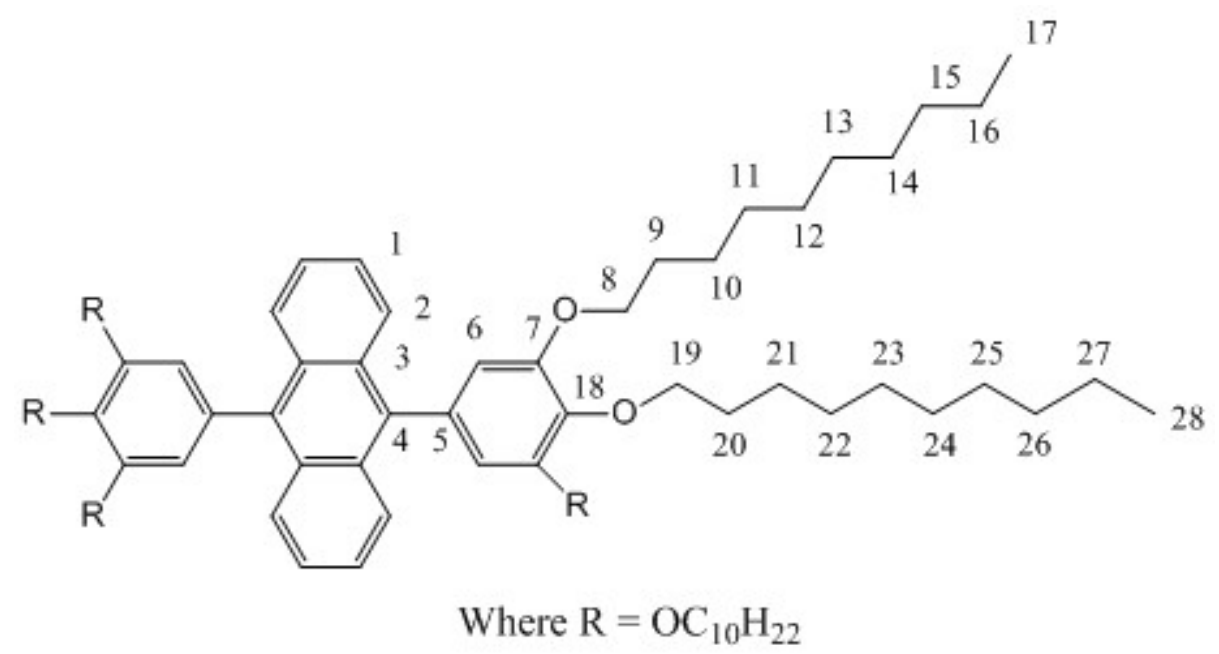

9,10-Bis(3,4,5-tri(hexadecyloxy)phenyl)anthracene (18). Following a similar procedure as described above, 25 (0.26 g, $0.51 \mathrm{mmol})$ was reacted with boron tribromide and then with bromohexadecane $(1.5 \mathrm{~mL}, 5$ mmol) affording the product which was purified by recrystallization from dichloromethane/ethanol. (Yield: $0.041 \mathrm{~g}, 5 \%){ }^{1} \mathrm{H}$ NMR $\delta\left(500 \mathrm{MHz}, \mathrm{CDCl}_{3}\right)$ : $7.75(\mathrm{~m}, \mathrm{~J}=10 \mathrm{~Hz}, 4 \mathrm{H}, \mathrm{H} 1), 7.35$ (m, J = $10 \mathrm{~Hz}, 4 \mathrm{H}, \mathrm{H} 2), 6.64$ (s, 4H, H6), $4.13(\mathrm{t}, \mathrm{J}=5 \mathrm{~Hz}, 4 \mathrm{H}, \mathrm{H} 25), 3.94$ (t, J = $10 \mathrm{~Hz}, 8 \mathrm{H}, \mathrm{H} 8), 1.87$ (q, 4H, H26), 1.8 (q, 8H, H9), 1.57-1.2 (m, 156H, H10-H22 and H27-H39), 0.87 (m, 18H, $\mathrm{H} 23$ and $\mathrm{H} 40) .{ }^{13} \mathrm{C}$ NMR $\delta\left(125 \mathrm{MHz}, \mathrm{CDCl}_{3}\right): 153.17$ (s, C7), 137.44 (s, C24), 134.01 and 129.91 (two singlets, C3 and C4), 127.20 (s, C1), 125.15 (s, C2), 110.15 (s, C6), 109.86 (s, C5), 73.72 (s, C25), 69.28 (s, C8), 14.27 (s, C23 and C40). Remaining $28 \mathrm{CH}_{2}$ carbon environments (C10-C22 and C27-C39) lie between 32.09 and 22.84. 


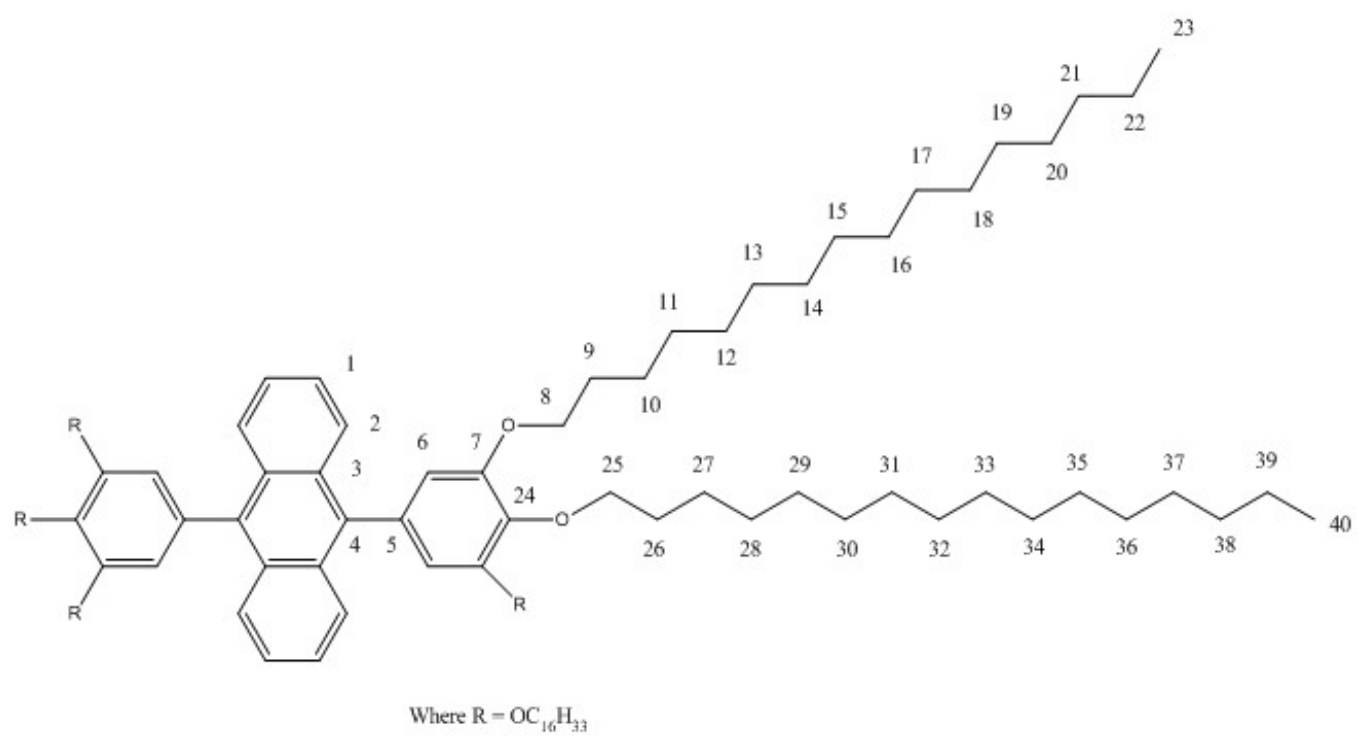

\subsubsection{Synthesis of Dicatenar BPEA Derivatives}

4-(Octyloxy)phenyl bromide (19). 4-Hydroxyphenyl bromide (2 g, 11.5 mmol), potassium carbonate $(2.8 \mathrm{~g}, 20.5 \mathrm{mmol})$, bromooctane $(2.97 \mathrm{~mL}$, $17.25 \mathrm{mmol})$ and acetone $(100 \mathrm{~mL})$ were combined and allowed to react at $50{ }^{\circ} \mathrm{C}$ for 6 days. The reaction mixture was poured into an excess of water and extracted with hexanes. This was further purified through column chromatography on silica using a solvent mix of $10 \%$ dichloromethane in hexanes to yield a colourless oil. (Yield: $1.91 \mathrm{~g}, 58.2 \%$ )

4-(Octyloxy)phenyltrimethylsilylacetylene (20). 19 (1.9 g, 6.69 $\mathrm{mmol})$, copper iodide $(0.064 \mathrm{~g}, 0.33 \mathrm{mmol})$, tetrakis(triphenylphosphine) palladium(0) (0.39 g, $0.33 \mathrm{mmol})$, di-isopropylamine $(1.2 \mathrm{~mL}, 8.5 \mathrm{mmol})$ and trimethylsilylacetylene $(1.2 \mathrm{~mL}, 8.5 \mathrm{mmol})$ were combined in dry toluene (20 $\mathrm{mL}$ ) and allowed to react for 5 days at $70^{\circ} \mathrm{C}$. The reaction mixture was diluted with dichloromethane, washed with a solution of ammonium chloride (saturated). The resulting organic layer was dried over anhydrous magne- 
sium sulfate, filtered and solvent removed in vacuo to yield a pale yellow oil. This was purified through column chromatography on silica using a solvent mix of $10 \%$ dichloromethane in hexanes. (Yield: $0.92 \mathrm{~g}, 61.6 \%$ )

4-(Octyloxy)phenylacetylene (21). 20 (0.49 g, $1.63 \mathrm{mmol})$ and potassium hydroxide $(0.86 \mathrm{~g}, 15.4 \mathrm{mmol})$ were com-bined in a solvent mixture of tetrahydrofuran : methanol : water $(4 \mathrm{~mL}: 4 \mathrm{~mL}: 1 \mathrm{~mL})$ and stirred overnight at room temperature. The reaction mixture was diluted with water and extracted with dichloromethane. The organic layer was dried over anhydrous magnesium sulfate, filtered and dried in vacuo to yield a orange oil. This was purified through column chromatography on silica using a solvent mix of $1 \%$ ethyl acetate in hexanes. (Yield: $0.29 \mathrm{~g}, 37.5 \%$ )

9,10-Bis(4-(octyloxy)phenylethynyl)anthracene (22). 21 (0.47 g, $2.02 \mathrm{mmol}), \mathbf{5}(0.31 \mathrm{~g}, 0.92 \mathrm{mmol})$, copper iodide $(0.02 \mathrm{~g}, 0.1 \mathrm{mmol})$, tetrakis (triphenylphosphine)palladium(0) (0.12 g, $0.1 \mathrm{mmmol})$, di-isopropylamine $(0.46 \mathrm{~mL}, 3.28 \mathrm{mmol})$ were combined in dry toluene $(20 \mathrm{~mL})$ and allowed to react for 5 days at $70^{\circ} \mathrm{C}$. Solvent was removed in vacuo, and crude purified by column chromatography on silica with using hexanes as the eluent. (Yield: $0.26 \mathrm{~g}, 20.1 \%{ }^{1} \mathrm{H}$ NMR $\delta\left(500 \mathrm{MHz}, \mathrm{CDCl}_{3}\right): 8.69(\mathrm{~m}, 4 \mathrm{H}, \mathrm{H} 1), 7.7$ (d, J $=9 \mathrm{~Hz}, 4 \mathrm{H}, \mathrm{H} 3), 7.62(\mathrm{~m}, 4 \mathrm{H}, \mathrm{H} 2), 6.97$ (d, J = 8.4 Hz, 4H, H4), 4.03 (t, J $=6.6 \mathrm{~Hz}, 4 \mathrm{H}, \mathrm{H} 5), 1.81$ (m, 4H, H6), 1.5-1.2 (m, 20H, H7 - H11), 0.87 (m, $6 \mathrm{H}, \mathrm{H} 12)$.

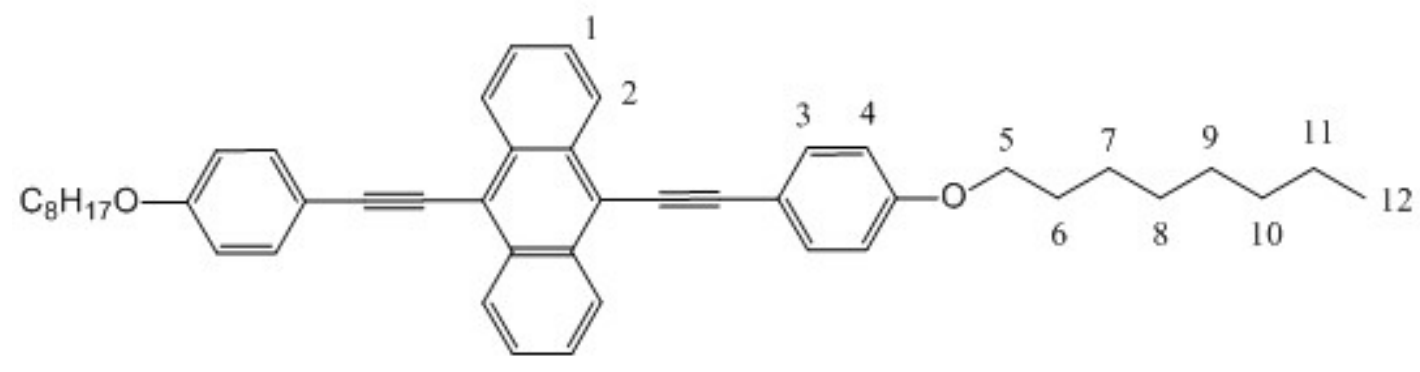


9,10-Bis(4-(hexadecyloxy)phenylethynyl)anthracene (23). This derivative was provided by Dr. K. Allan, synthesized using similar synthetic methods to that of the octyl derivative above. ${ }^{69}{ }^{1} \mathrm{H}$ NMR $\delta(500 \mathrm{MHz}$, $\left.\mathrm{CDCl}_{3}\right): 8.69(\mathrm{~m}, 4 \mathrm{H}, \mathrm{H} 1), 7.7$ (d, J = 5.4 Hz, 4H, H3), 7.62 (m, 4H, H2), $6.98(\mathrm{~d}, \mathrm{~J}=5.1 \mathrm{~Hz}, \mathrm{H} 4), 4.03$ (t, J = $3.9 \mathrm{~Hz}, 4 \mathrm{H}, \mathrm{H} 5), 1.89$ (m, 4H, H6), 1.5 - 1.2 (m, 52H, H7-H19), 0.88 (m, 6H, H20).

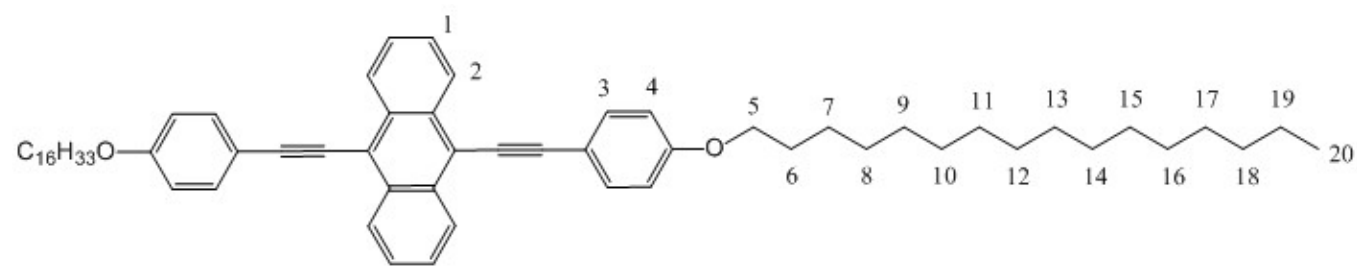

9,10-Bis(4'-(octyloxy)biphenyl-4-ylethynyl)anthracene (24). This derivative was provided by Dr. K. Allan, its synthesis was based on literature methods. $\underline{69}{ }^{1} \mathrm{H}$ NMR $\delta\left(500 \mathrm{MHz}, \mathrm{CDCl}_{3}\right): 8.73(\mathrm{dd}, \mathrm{J}=7 \mathrm{~Hz}, 4 \mathrm{H}, \mathrm{H} 1)$, $7.83(\mathrm{~d}, \mathrm{~J}=10 \mathrm{~Hz}, 4 \mathrm{H}, \mathrm{H} 3), 7.68-7.65$ (m, 8H, H5-H6), 7.6 (d, J = $9 \mathrm{~Hz}$, 4H, H2), 7.02 (d, J = $9 \mathrm{~Hz}, 4 \mathrm{H}, \mathrm{H} 4), 4.03$ (t, J =6 Hz, 4H, H7), 1.84 (q, 4H, H8), 1.49 (m, 4H, H9), 1.4-1.2 (m, 16H, H9-12), 0.89 (m, 6H, H14).

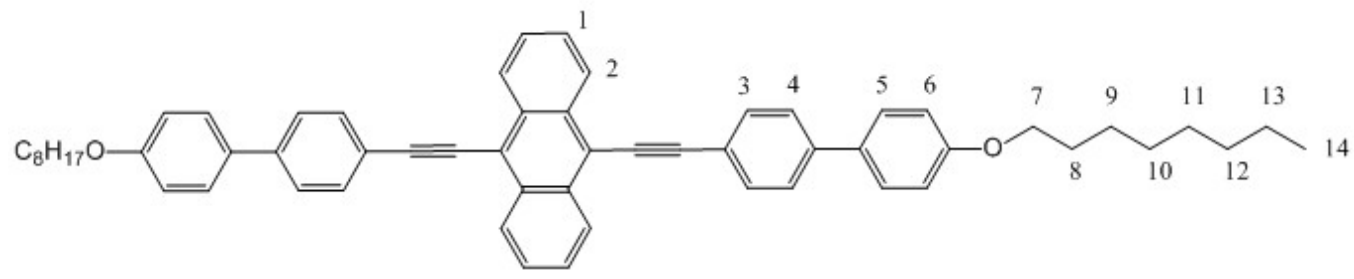




\subsection{Liquid Crystal Techniques}

As discussed in chapter 3, both differential scanning calorimetry and polarised optical microscopy are techniques used together to ascertain the phases formed by the material, and if a mesophase is also formed.

\subsubsection{Differential Scanning Calorimetry (DSC)}

This technique compares the intake of heat in a sample compared to an inert reference as a function of temperature $\left(\right.$ Fig. 8.1). ${ }^{40 \mid 41}$ As a phase transition occurs energy is either given out or taken in by the sample and this is represented by a peak in the DSC at this temperature, known as the 'heat of transition'. The programme of heating and cooling the sample can be varied to elucidate the temperatures at which the phase transitions occur. This technique was used to identify these temperatures, however it did not provide information as to which liquid crystalline phases were formed, only the mesophase temperatures necessary for POM investigations. This is a very sensitive technique in which the heating rate, temperature range and hold times were adjusted for complete elucidation of transition temperatures.

A Shimadzu DSC-60 calibrated with metallic indium supplied by MettlerToledo was used. Samples of between 5-10 mg were analysed in crimped aluminium pans, and measured against an empty aluminium reference pan in a nitrogen atmostphere, and the data processed using Shimadzu TA-60 thermal data analysis software.

In the case of the decyl hexacatenar DPA derivative, that measurement was performed on a Thermal Associates DSC-Q100, which can cool to temperatures up until $-80^{\circ} \mathrm{C}$. 


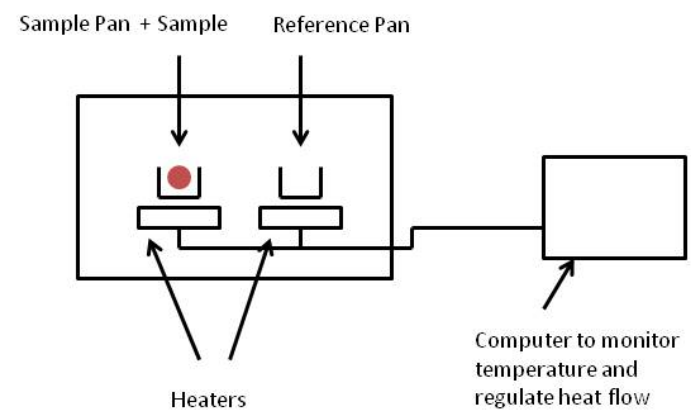

Fig. 8.1 A schematic of the operation of a DSC machine.

\subsubsection{Polarised Optical Microscopy (POM)}

This technique exploits the birefringent properties and defects of liquid crystal textures. $\frac{4143}{4 i r e f r i n g e n c e ~ h o w e v e r ~ i s ~ n o t ~ l i m i t e d ~ t o ~ l i q u i d ~ c r y s t a l l i n e ~}$ phases, it is also found in many crystalline solids. It is the defects associated with high ordering within the phases which give rise to characteristic textures which allows the viewer to determine mesomorphic behaviour.

In this technique, the sample was placed between two cross-polarised filters and heated to the required temperatures on a hot stage. ${ }^{43}$ The incident light is polarised as it passes through the first filter which then travels through the sample (Fig. 8.2). Liquid crystals exhibit birefringence as they have multiple refractive indices due to their directionally dependent (anisotropic) 
properties. ${ }^{40 \mid 41 / 43}$ This causes the extraordinary wave to be slowed or sped up as it passes through the sample, causing it to oscillate out of phase with the magnetic wave giving circularly polarised light. As this light is passed through the analyser (which is rotated $90^{\circ}$ compared to the polariser), wavelengths that have been rotated are seen, but light that is unchanged appears dark. If this is a liquid crystal phase, due to the high anisotropy, certain textures will be observed. 14

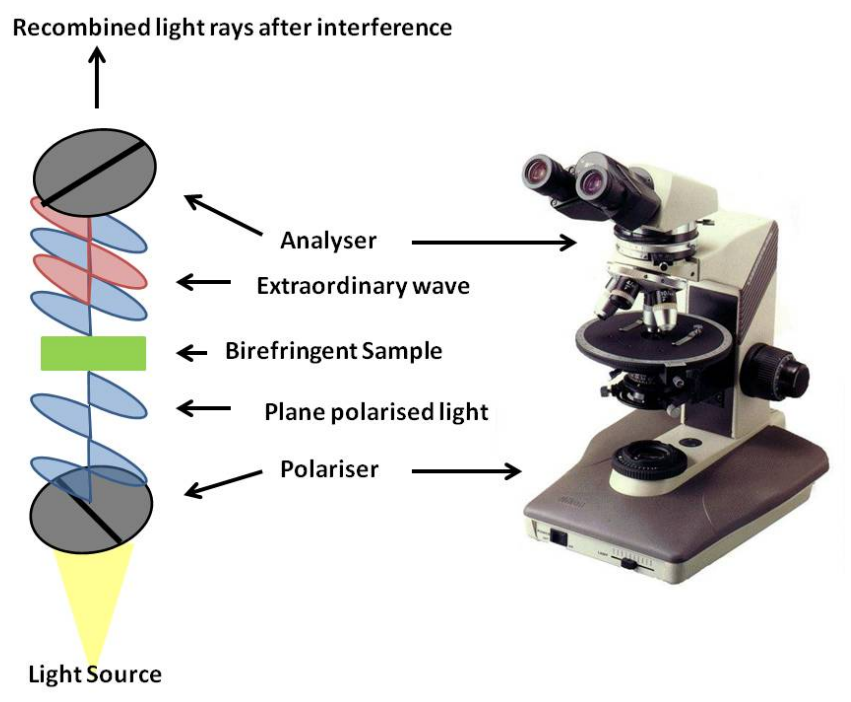

Fig. 8.2 A schematic of the mechanics of a polarised optical microscope, with the polariser and analyser at $90^{\circ}$.

A Nikon Optishot-2 polarising microscope, fitted with a Nikon D200 DSLR 10.3 megapixel camera was used. Samples were prepared by placing a small amount of sample (mg) on a slide and covering with a cover slip. The temperature of the sample was adjusted by using a Mettler-Toledo FP82HT hot stage.

It should be noted that temperatures may vary between those very precisely and carefully measured in the DSC, compared to those more crudely measured by heating and cooling on a hot stage under the microscope, where heat transfer may be less efficient, and temperature monitoring less precise. 


\subsection{Cholesteryl Benzoate - A Worked Exam- ple}

In order to demonstrate how the techniques defined above; DSC and POM, will be used to elucidate the phase behaviour or presence of phase behaviour of the molecules synthesized in this thesis, the phase behaviour of a known liquid crystal, cholesteryl benzoate was investigated (Fig. 8.3) ${ }^{38}$ This material and its derivatives, have well known and defined liquid crystalline behaviour. $\underline{40141}$ They are considered a special class of liquid crystal, which form mainly twisted nematic phases, which are termed 'cholesteric' phases. ${ }^{41}$

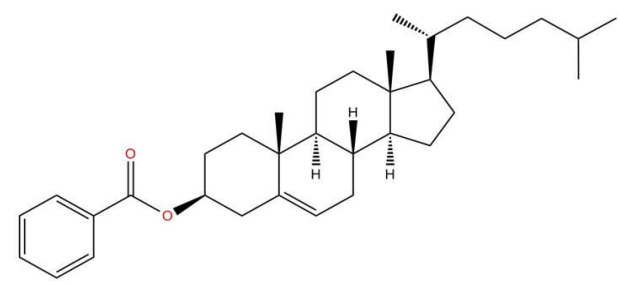

Fig. 8.3 Structure of cholesteryl benzoate.

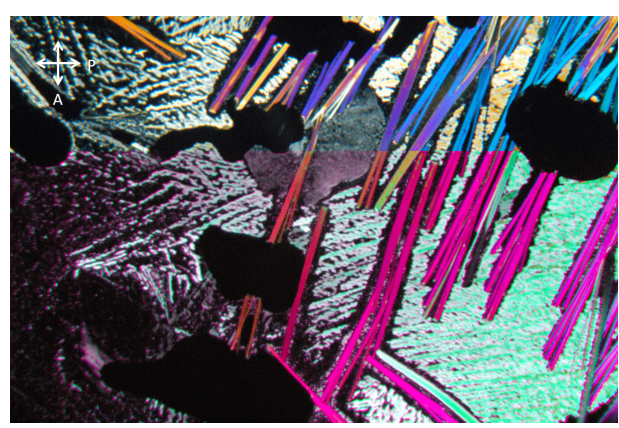

Fig. 8.4 Image of the needle like crystals of cholesteryl benzoate at $130{ }^{\circ} \mathrm{C}$ at 200x Magnification.

At room temperature, the sample consisted of needle shaped birefringent crystals (Fig. 8.4) and upon heating and cooling formed two liquid crystalline 
phases.

On heating a single peak at $140.95^{\circ} \mathrm{C}$, with an enthalpy of $-34.19 \mathrm{Jg}^{-1}$ was observed in the DSC trace, which corresponded to the presence of a smectic A phase. This was evidenced by observation under the microscope by circular focal conic domains, with a disinclination strength of -1 (Fig. 8.5). The temperature is close to that observed by Reinitzer at $145.5^{\circ} \mathrm{C} . \frac{38}{\text { It }}$ is common for strongly twisted cholesteric derivatives to form phases with a quasi layered structure, similar to smectic states. ${ }^{41}$ The formation of the isotropic liquid was not seen in the DSC due to decomposition of the sample at temperatures higher than $145^{\circ} \mathrm{C}$.
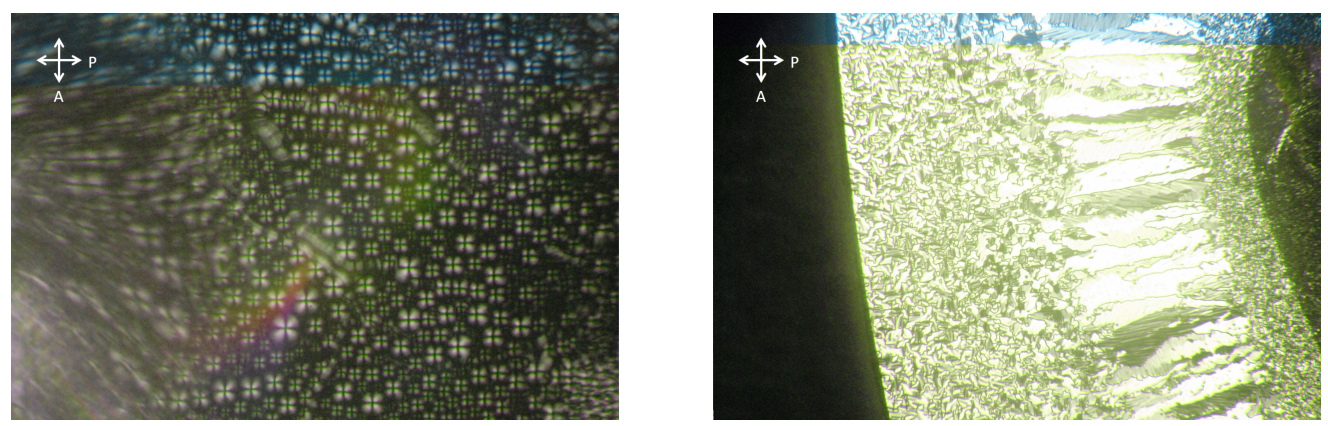

Fig. 8.5 POM images of smectic (left) at $146^{\circ} \mathrm{C}$ and cholesteric (right) phases at $120.9^{\circ} \mathrm{C}$ of cholesteryl benzoate at $200 \mathrm{x}$ magnification.

Upon cooling, a single peak was observed in the DSC trace at $124.17^{\circ} \mathrm{C}$, with an enthalpy of $39.63 \mathrm{Jg}^{-1}$ corresponding to the crystallisation of the sample. However, under the microscope formation of a cholesteric phase with a fan shaped texture was observed upon cooling from the isotropic liquid at $157.3-149^{\circ} \mathrm{C}$ (Fig. 8.5). This was not seen in the DSC trace due to the presence of noise in the baseline. 


\subsection{Spectroscopic Methods}

All absorption and emission solution samples were diluted to the appropriate concentration, in spectroscopic grade toluene. All absorption spectra were obtained on a Agilent diode array, baseline corrected, UV-vis spectrophotometer, with two-sided quartz cuvettes unless otherwise stated. All emission spectra were obtained on a Shimadzu RF-5301PC Spectrofluorophotometer, with four-clear sided quartz cuvettes unless otherwise stated.

Visual observation of upconversion in solution, power dependence and emission of PdOEP/BPEA solution were obtained using the following experimental set up. A $6 \mathrm{~W} 532 \mathrm{~nm}$ laser was used for the excitation source (Laser Glow Technologies, with a Brightline AC power supply). This was focused using a large convex lens onto the sample in a cuvette. The cuvette was a quartz fluorescence cuvette equipped with a tap. The upconverted fluorescence was collected using a small focusing lens and USB optical fiber spectrometer (Spectral Products, SM442-USB optical fiber).

For all absorption and emission measurements of thin films, the above instruments were used for both absorption and emission spectra unless otherwise stated. 


\section{References}

1. Islangulov, R. R.; Lott, J.; Weder, C.; Castellano, F. N. Journal of the American Chemical Society 2007, 129, 12652.

2. Honsberg Bowden, S.,, C. ASTN G-173-03. 1992; http://pveducation. org/pvcdrom/appendices/standard-solar-spectra.

3. Shockley, W.; Queisser, H. J. Journal of Applied Physics 1961, 32, 510.

4. Singh-Rachford, T. N.; Castellano, F. N. Coordination Chemistry Reviews 2010, 254, 2560.

5. University of California Davis Chemwiki. Dexter Energy Transfer. http://chemwiki.ucdavis.edu/Theoretical_Chemistry/ Fundamentals/Dexter_Energy_Transfer (accessedApril19, 2015).

6. Monguzzi, A.; Mezyk, J.; Scotognella, F.; Tubino, R.; Meinardi, F. Physical Review B 2008, 78, 195112.

7. Luo, Y.; Li, D.; Meng, Q. Advanced Materials 2009, 21, 4647.

8. Giménez, R.; Piñol, M.; Serrano, J. L. Chemistry of Materials 2004, 16, 1377.

9. Zhang, Z.; Tang, H.; Wang, H.; Liang, X.; Liu, J. et al. Thin Solid Films 2007, 515, 3893.

10. Kozlov, D. V.; Castellano, F. N. Chemical communications 2004, 2860.

11. Wilson, G. J.; Launikonis, A.; Sasse, W. H. F.; Mau, A. W.-H. The Journal of Physical Chemistry A 1997, 101, 4860. 
12. Weinheimer, C.; Choi, Y.; Caldwell, T.; Gresham, P.; Olmsted, J. Journal of Photochemistry and Photobiology A: Chemistry 1994, 78, 119.

13. Tyson, D. S.; Bignozzi, C. A.; Castellano, F. N. Journal of the American Chemical Society 2002, 124, 4562.

14. Tsvirko, M. P.; Stelmakh, G. F.; E. Pyatosin, V.; Solovyov, K. N.; Kachura, T. F. Chemical Physics Letters 1980, 73, 80.

15. Islangulov, R. R.; Kozlov, D. V.; Castellano, F. N. Chemical communications 2005, 3776 .

16. Baluschev, S.; Yakutkin, V.; Miteva, T.; Wegner, G.; Roberts, T. et al. New Journal of Physics 2008, 10, 013007.

17. Monguzzi, A.; Tubino, R.; Meinardi, F. The journal of physical chemistry. A 2009, 113, 1171.

18. Singh-Rachford, T. N.; Islangulov, R. R.; Castellano, F. N. The journal of physical chemistry. A 2008, 112, 3906.

19. Wang, X.; Del Guerzo, A.; Schmehl, R. H. Journal of Photochemistry and Photobiology C: Photochemistry Reviews 2004, 5, 55.

20. Förster, T. Annalen der Physik 1948, 437, 55.

21. Dexter, D. L. The Journal of Chemical Physics 1953, 21, 836.

22. Monguzzi, A.; Tubino, R.; Meinardi, F. Physical Review B 2008, 77, 155122.

23. Simon, Y. C.; Weder, C. Journal of Materials Chemistry 2012, 22, 20817.

24. Parker, C. A.; Hatchard, C. G.; Joyce, T. A. Journal of Molecular Spectroscopy 1964, 14, 311.

25. Parker, C. A.; Hatchard, C. G.; Joyce, T. A. Nature 1965, 205, 1282.

26. van Sark, W.; de Wild, J.; Rath, J.; Meijerink, A.; Schropp, R. E. I. Nanoscale Research Letters 2013, 8, 81.

27. Singh-Rachford, T. N.; Lott, J.; Weder, C.; Castellano, F. N. Journal of the American Chemical Society 2009, 131, 12007. 
28. Heller, C. A.; Henry, R. A.; McLaughlin, B. A.; Bliss, D. E. Journal of Chemical \&f Engineering Data 1974, 19, 214.

29. Baluschev, S.; Miteva, T.; Yakutkin, V.; Nelles, G.; Yasuda, a. et al. Physical Review Letters 2006, 97, 143903.

30. Haefele, A.; Blumhoff, J.; Khnayzer, R. S.; Castellano, F. N. The Journal of Physical Chemistry Letters 2012, 3, 299.

31. Merkel, P. B.; Dinnocenzo, J. P. Journal of Luminescence 2009, 129, 303.

32. Moor, K.; Kim, J.-H.; Snow, S.; Kim, J.-H. Chemical communications 2013, 49, 10829.

33. Cicerone, M. T.; Blackburn, F. R.; Ediger, M. D. The Journal of Chemical Physics 1995, 102, 471.

34. Maulding, D. R.; Roberts, B. G. The Journal of Organic Chemistry $1969,34,1734$.

35. de Gennes, P.; Prost, J.; Pelcovits, R. Physics Today 1995, 48, 70.

36. Sluckin, T. J.; Dunmur, D. A.; Stegemeyer, H. Crystals that flow: Classic papers from the history of liquid crystals; CRC Press: Florida, 2004; p 512.

37. Mery, S.; Haristoy, D.; Nicoud, J.-F.; Guillon, D.; Monobe, H. et al. Journal of Materials Chemistry 2003, 13, 1622.

38. Reinitzer, F. Monatshefte für Chemie 1888, 9, 421.

39. Fisch, M. R. Liquid crystals, laptops and life; World Scientific: Singapore, 2004; Vol. 23; p 376.

40. Collings, P. J.; Hird, M. Introduction to liquid crystals: chemistry and physics; CRC Press: Florida, 1997; p 312.

41. Demus, D.; Richter, L. Textures of Liquid Crystals; Weinheim: New York, 1978; p 228.

42. Brown, G. H., Ed. Advances in Liquid Crystals Vol. 2; Academic Press: United States of America, 2013; p 320. 
43. Murphy, D. Fundamentals of Light Microscopy and Electronic Imaging; Wiley-Liss: New York, 2001; p 384.

44. Uchimura, M.; Ishige, R.; Arakawa, Y.; Niko, Y.; Watanabe, J. et al. Research on Chemical Intermediates 2013, 39, 403.

45. Yamane, S.; Sagara, Y.; Mutai, T.; Araki, K.; Kato, T. Journal of Materials Chemistry C 2013, 1, 2648.

46. Yamane, S.; Sagara, Y.; Kato, T. Chemical Communications 2013, 49, 3839.

47. Olivier, J.-H.; Camerel, F.; Barberá, J.; Retailleau, P.; Ziessel, R. Chemistry A European Journal 2009, 15, 8163.

48. Chanishvili, A.; Chilaya, G.; Petriashvili, G.; Barberi, R.; Bartolino, R. et al. Advanced Materials 2004, 16, 791.

49. Sagara, Y.; Yamane, S.; Mutai, T.; Araki, K.; Kato, T. Advanced Functional Materials 2009, 19, 1869.

50. Zhu, J.; Zhong, K.; Liang, Y.; Wang, Z.; Chen, T. et al. Tetrahedron 2014, 70, 1230 .

51. Yasuda, T.; Shimizu, T.; Liu, F.; Ungar, G.; Kato, T. Journal of the American Chemical Society 2011, 133, 13437.

52. Furst, M.; Kallmann, H.; Brown, F. H. The Journal of Chemical Physics 1957, 26, 1321.

53. Nakatsuji, S.; Matsuda, K.; Uesugi, Y.; Nakashima, K.; Akiyama, S. et al. Journal of the Chemical Society, Perkin Transactions 1 1992, 7, 755 .

54. Nguyen, P.; Todd, S.; Van den Biggelaar, D.; Taylor, N. J.; Marder, T. B. et al. Synlett 1994, 4, 299.

55. Twieg, R. J.; Chu, V.; Nguyen, C.; Dannels, C. M.; Viney, C. Liquid Crystals 2006, 20, 287.

56. Littke, A. F.; Dai, C.; Fu, G. C. Journal of the American Chemical Society 2000, 122, 4020. 
57. Hundertmark, T.; Littke, A. F.; Buchwald, S. L.; Fu, G. C. Organic letters 2000, 4.

58. Div, M.; Littke, A. F.; Fu, G. C. Angewandte Chemie 1998, 2, 3387.

59. Lincker, F.; Bourgun, P.; Masson, P.; Didier, P.; Guidoni, L. et al. Organic letters 2005, 7, 1505.

60. Li, W.-S.; Yamamoto, Y.; Fukushima, T.; Saeki, A.; Seki, S. et al. Journal of the American Chemical Society 2008, 130, 8886.

61. Sakurai, T.; Shi, K.; Sato, H.; Tashiro, K.; Osuka, A. et al. Journal of the American Chemical Society 2008, 130, 13812.

62. Schultz, A.; Laschat, S.; Diele, S.; Nimtz, M. European Journal of Organic Chemistry 2003, 2003, 2829.

63. Maeda, H.; Terashima, Y.; Haketa, Y.; Asano, A.; Honsho, Y. et al. Chemical communications 2010, 46, 4559 .

64. Kim, Y. H.; Shin, D. C.; Kim, S.-H.; Ko, C.-H.; Yu, H.-S. et al. Advanced Materials 2001, 13, 1690.

65. Audrieth, L. F., Ed. Inorganic Syntheses Vol. III; McGraw-Hill Book Company Inc.: United States of America, 1950; p 300.

66. Brauer, G.; Becher, H. J. Handbook of preparative inorganic chemistry; Academic Press: London, 1963; p 1906.

67. Duan, P.; Yanai, N.; Kimizuka, N. Journal of the American Chemical Society 2013, 135, 19056.

68. Babu, S. S.; Hollamby, M. J.; Aimi, J.; Ozawa, H.; Saeki, A. et al. Nature communications 2013, 4, 1969.

69. Allan, K. M. Unpublished Work; 2014.

70. Uchimura, M.; Kang, S.; Ishige, R.; Watanabe, J.; Konishi, G. Chemistry Letters 2010, 39, 513.

71. Kelley, C. J.; Ansu, K.; Budisusetyo, W.; Ghiorghis, A.; Qin, Y. et al. Journal of Heterocyclic Chemistry 2001, 38, 11. 
72. Grewer, C.; Brauer, H.-D. The Journal of Physical Chemistry 1994, 98, 4230.

73. Mitsui, M.; Kawano, Y.; Takahashi, R.; Fukui, H. RSC Advances 2012, 2, 9921. 